Review

\title{
Recent Developments and Applications of Solid Phase Microextraction (SPME) in Food and Environmental Analysis-A Review
}

\section{Sybille Merkle ${ }^{1, \dagger}$, Kim Karen Kleeberg ${ }^{2, \dagger}$ and Jan Fritsche ${ }^{1, *}$}

1 Faculty Life Sciences, Department Nutrition \& Home Economics/Food Science, Hamburg University of Applied Sciences, Ulmenliet 20, 21033, Hamburg, Germany; E-Mail: sybille.merkle@haw-hamburg.de

2 ODOCON Dr. Kim Kleeberg \& Dr. Jan Streese-Kleeberg GbR, Amtsstraße 7, 22143, Hamburg, Germany; E-Mail: info@odocon.de

$\dagger$ These authors contributed equally to this work.

* Author to whom correspondence should be addressed; E-Mail: jan.fritsche@haw-hamburg.de; Tel.: +49-40-42875-6163; Fax: +49-40-42875-6205.

Academic Editor: Mary Boyce

Received: 1 April 2015 / Accepted: 11 June 2015 / Published: 30 June 2015

\begin{abstract}
Solid-phase microextraction (SPME) is a simple, sensitive, rapid and solvent-free technique for the extraction of analytes from gaseous, liquid and solid samples and takes a leading position among microextraction methods. Application of SPME in sample preparation has been increasing continuously over the last decade. It is most often used as an automatized fiber injection system coupled to chromatographic separation modules for the extraction of volatile and semivolatile organic compounds and also allows for the trace analysis of compounds in complex matrices. Since SPME was first introduced in the early 1990s, several modifications have been made to adapt the procedure to specific application requirements. More robust fiber assemblies and coatings with higher extraction efficiencies, selectivity and stability have been commercialized. Automation and on-line coupling to analytical instruments have been achieved in many applications and new derivatization strategies as well as improved calibration procedures have been developed to overcome existing limitations regarding quantitation. Furthermore, devices using tubes, needles or tips for extraction instead of a fiber have been designed. In the field of food analysis, SPME has been most often applied to fruit/vegetables, fats/oils, wine, meat products, dairy and
\end{abstract}


beverages whereas environmental applications focus on the analysis of air, water, soil and sediment samples.

Keywords: SPME; food analysis; environmental analysis; volatile compounds; aroma; off-flavor; GC; LC; SBSE

\section{Introduction}

The quality of trace analysis in food and environmental samples mainly depends on the selected sample treatment [1]. There is an increasing demand for strategies minimizing the analyte-matrix interferences, especially in case of volatile compounds occurring at low concentrations in complex matrices. These conditions require the reduction of final volumes of analytes to furnish higher concentrations of analytes at low quantification limits [2]. Moreover, there is a demand for environmentally sustainable procedures that apply reduced volumes of organic solvents [2]. Another aspect of analytical method development is automation [3]. There are numerous advantages of automation, e.g., lower time consumption, increase of simplicity, lower probability of sample contamination and higher repeatability. These are just a few reasons for the widespread application of solid phase microextration (SPME) techniques [3].

SPME is a relatively recent and easy to automate technique for the extraction of analytes from gaseous, liquid, and solid matrices. The simple, rapid and solvent-free technique was introduced in the early 1990s by Pawliszyn and coworkers [4-6]. The SPME process is composed of two basic steps: (i) partitioning of analytes between the extraction phase and the sample matrix and (ii) desorption of concentrated extracts into an analytical instrument [7]. Due to the combination of sampling, extraction, pre-concentration and sample introduction into an analytical instrument in one single step, SPME has gained popularity in many fields of application in recent years, especially in routine laboratories and industrial applications [8-10]. Areas of application have been steadily growing, including food packaging and environmental uses.

Automatised fiber injection systems hyphenated with gas chromatographic (GC) and gas chromatography-mass spectrometry (GC-MS) separation modules are the most popular instruments combined with SPME in use [11]. These instrumental assemblies have been successfully applied to a wide variety of compounds, especially for the extraction of volatile and semivolatile organic compounds from complex sample matrices [12]. Due to the inaccessibility of weakly volatile or thermally labile compounds to GC or GC-MS, SPME was also developed for direct coupling to high-performance liquid chromatography (HPLC) and liquid chromatography-mass spectrometry (LC-MS) [12].

A number of reviews on SPME have already been published, dealing with food analysis [12-15], environmental analysis [16-21], calibration methods [22], SPME coatings [11,23-26], drug analysis [12,27], the analysis of specific compounds [28,29] and specific SPME techniques [30]. In addition, general overviews of SPME techniques and contemporary developments are provided by others [2,6,17,31].

This review provides a comprehensive overview on latest trends of SPME method development including new techniques and new devices, as well as essential parameters in SPME processes. The latest development of fiber coating development and the application of nanotechnology in SPME 
technology are also covered extensively by the present review. Moreover, this review reports for the first time simultaneously on two important life sciences SPME application areas-food and environmental applications published over the last decade (2005-2015).

\section{SPME Techniques}

The underlying principle of the SPME methodology is based on the partitioning of analytes between a coated fiber and a sample. The reusable fiber is coated with a stationary phase, which can be a liquid polymer, a solid sorbent, or a combination of both [9]. A mass transfer begins after exposure to the vapour phase above a solution (HS-SPME) or direct immersion in the solution (DI-SPME). This process is driven by the second law of thermodynamics. Thereby the chemical potential of each compound should be equal. Once equilibrium is reached, the extracted compounds on the fiber are desorbed and inserted into the injection port of a chromatograph. The release of the analytes is accomplished in GC by thermal desorption and in HPLC by dissolution and subsequent injection of the elution solvent [32]. Both methods lead to detection of the substances by the analytical instrument detector. The application of SPME-LC has lagged behind SPME-GC, which might be due to the small number of commercially available SPME sorbents for LC applications, the lack of commercially available interfaces, long equilibration times, and the lack of automation [2].

The aim of SPME is to reach the equilibrium between the sample matrix and the coating of the SPME device as rapidly as possible [33]. Further exposure of the fiber after equilibrium has been reached does not increase the concentration of extracted compounds. For that reason, sample extraction and pre-concentration could be carried out in one single step [34].

SPME has several advantages over traditional extraction methods. It is not just a rapid, simple and solvent-free method, but it is also sensitive, provides linear results for a wide range of concentrations and analytes [2]. Notwithstanding the low concentrations of analytes, quantitative or semi-quantitative data are provided and losses that can occur during the extraction, concentration and clean-up steps of traditional sample procedures are mostly avoidable [2].

One of the main drawbacks of SPME techniques is the limited number of commercially available stationary phases (fiber materials) only roughly covering the scale of polarity of target analytes. In particular, the extraction of polar analytes from samples with a polar matrix poses a problem [35-39]. Other challenges are the relatively low recommended operating temperature $\left(240-280{ }^{\circ} \mathrm{C}\right)$, the instability and swelling in organic solvents, breakage of the fiber, stripping of coatings, bending of the needle, and the cost as well as the limited lifetime of the fiber [2,36]. Furthermore, sample carry-over may occur [40] and high molecular weight compounds cannot be analyzed in combination with GC [36]. In certain cases, low extraction efficiencies are reported, in particular in case of very volatile, polar, or thermally unstable analytes [2]. The early theoretical and practical aspects of SPME have undergone continuous technical development over the years.

SPME techniques cover a wide range of sampling techniques, including field, in situ and air sampling. The two different implementations fiber SPME and in-tube SPME are frequently used [9]. Detailed SPME theory has been explained in a number of reviews $[6,15,41,42]$. 


\subsection{Fiber Solid-Phase Microextraction}

"Fiber"-SPME devices have been developed and currently represent the widespread SPME technique which comprises a fiber holder and a fiber assembly [41]. The fiber assembly consists of a 1- to 2-cm long retractable SPME fiber [41] and a built-in coated fiber that looks like a modified syringe [6,33]. After the sample is placed in a vial sealed with a septum-type cap, the SPME needle is pierced through the septum and the fiber is extended into the vial. The analytes partition between the sample matrix and the extraction phase until equilibrium of concentrations is reached. The maximum sensitivity is achieved and a proportional relationship is obtained between the amount of the extracted analyte by the SPME fiber and its initial concentration in the sample $[43,44]$.

\subsection{In-tube Solid-Phase Microextraction}

The more recent in-tube (IT) SPME was developed by Eisert and Pawliszyn [45] for application with HPLC or liquid chromatography (LC)-MS because fiber SPME could barely withstand aggressive HPLC solvent conditions [6,45]. In-tube SPME overcomes fiber-related drawbacks such as fragility, low sorption capacity and bleeding from thick-film coatings. It uses an open tubular fused-silica capillary in which the extraction phase is either an inner surface coating or a sorbent bed. Consequently, in-tube SPME devices exhibit considerably higher mechanical stability than fiber SPME devices. Another reason for the development of in-tube SPME was the lack of automation in the use of SPME coupled to HPLC [2]. By means of automation it is possible to perform extraction, desorption and injection simultaneously $[6,12]$. The advantages of automated systems compared to manual techniques are shorter total analysis times as well as higher accuracy and precision [2]. A drawback of in-tube SPME is the tendency of the capillary to clog up. This can be avoided by working with samples without interfering phases like particles or macromolecules [2]. Moreover, the enrichment factor is reduced compared to fiber SPME [2]. Kataoka [30] and Globig and Weickhardt [46] provided an overview of automated sample preparation using in-tube SPME and applications of this technique in environmental, clinical and food analysis, mainly for the determination of polar and thermolabile compounds.

In-tube SPME can be categorized into methods using extraction coatings where the coating is used as an internal extraction phase immobilized in the capillary wall and methods using extraction fillings where the extraction phase is a sorbent packing, as demonstrated in Figure 1 [47].

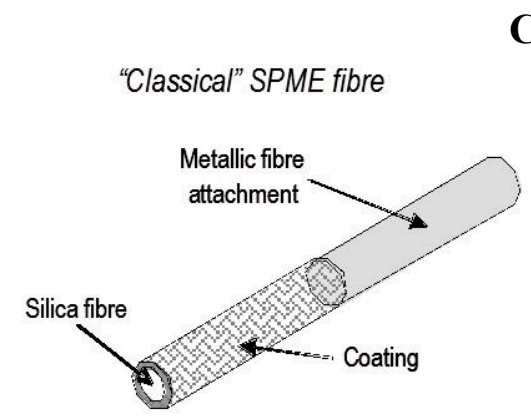

\section{Classical and in-tube SPME devices}

ITE with extraction coating

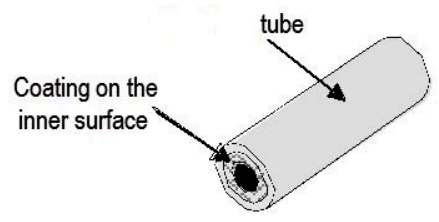

ITE with extraction filling

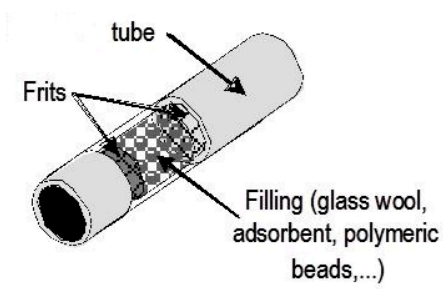

Figure 1. "Classical" solid-phase microextraction (SPME) fiber compared with in-tube fibers, adapted from Nerín et al. (2009) [2], reprinted with permission of Springer Verlag. 
The latter techniques are particularly similar to the previously developed solid-phase extraction (SPE) and use different sorbent types. Both in-tube SPME techniques can be easily applied with existing autosampler systems. Several in-tube devices with extraction fillings have been developed, firstly in 2004 by Kubinek et al. [48], and are now commercially available, for example as in-tube extraction (ITEX) [2].

There are two modes of extraction in in-tube SPME. In the static mode, the analytes are transferred to the stationary phase by mere diffusion, while in the dynamic mode, the sample is actively drawn through the tube by repeated draw/eject cycles [49]. When the equilibrium is virtually reached, the extracted analytes are desorbed from the capillary either by direct transfer to the liquid chromatographic column or by a stream of the mobile phase. In case of a stronger attachment of the analytes to the capillary, desorption is achieved by a static desorption solvent which is subsequently injected into the chromatographic system [50]. The desorbed analytes are carried through the column and separated and detected by a mass selective detector [9].

Even though in-tube SPME was originally developed for HPLC applications, it can also be used with other instrumental equipment, for example capillary electrophoresis (CE) or GC [30]. On-line coupling to GC can be achieved by open-tubular trapping (OTT), which is mainly applied to HS samples [30]. In OTT, desorption of the analytes is carried out with a small amount of solvent or by thermal desorption. However, OTT is characterized by a complex instrumental setup and unfavorable sampling conditions, such as high pressure drop from long traps and limited flow rates [2].

In-tube SPME techniques have been developed further and modifications like wire-in-tube and fiberin-tube techniques have emerged [51]. The wire-in-tube technique contains an additional stainless steel wire inserted into the extraction capillary. Thereby the surface of the coating material stay more or less the same, but the internal volume of the capillary is significantly reduced. A change in the phase ratio takes place which leads to preconcentration of the sample. This results in a more effective extraction [51]. In the fiber-in-tube technique, the extraction phase comprises several hundreds of delicate filaments of polymeric material packed longitudinally into a short capillary. This composition leads to enhanced pre-concentration of analytes [9].

\subsection{Cooled Coated Fiber Device}

Apart from in-tube SPME, procedures using a cooled fiber and other methods that do not necessarily rely on the use of a fiber have emerged. These new developments will be explained in the following section.

A recent development in the application of HS-SPME is an internally cooled coated fiber device (CCF) or cold fiber HS-SPME device. The main reason for developing this method was the improved release of analytes from the interfering phases in complex matrices [52,53].

For desorption of analytes from solid particles it is often useful to increase the extraction temperature. However absorption of analytes by the fiber coating is an exothermic process, therefore this process leads to a decrease of the partition coefficients [2]. The aim is to accelerate the mass transfer process and simultaneously increase the distribution constants of analytes [2]. For that purpose, an internally cooled coated fiber device was developed heating the sample matrix at the same time as the fiber coating is cooled down. Especially for matrices with high viscosity or for volatiles with low partition coefficients CCF is useful [2]. This technology was successfully employed for the first time to extract analytes from 
various environmental matrices [52-54]. CCF was also successfully applied to food analysis, such as extracting volatiles and semivolatiles from tropical fruit pulps by Carasek and Pawliszyn (2006). They stated that the cold fiber was the most suitable fiber for the purpose of extracting volatile compounds from the five fruit pulps studied [55]. Additionally, it was reported that cold-fiber HS-SPME offers more sensitivity and higher sample throughput than conventional HS-SPME [56]. One drawback, however, of the increased fiber capacity through this cooled coated fiber is the loss of selectivity [2]. Not only the analytes but also the interferences are extracted exhaustively onto the coating [6]. CCF has been miniaturized and automated first by Chen and Pawliszyn (2006) [53] and is nowadays routinely used (see Figure 2) [2].
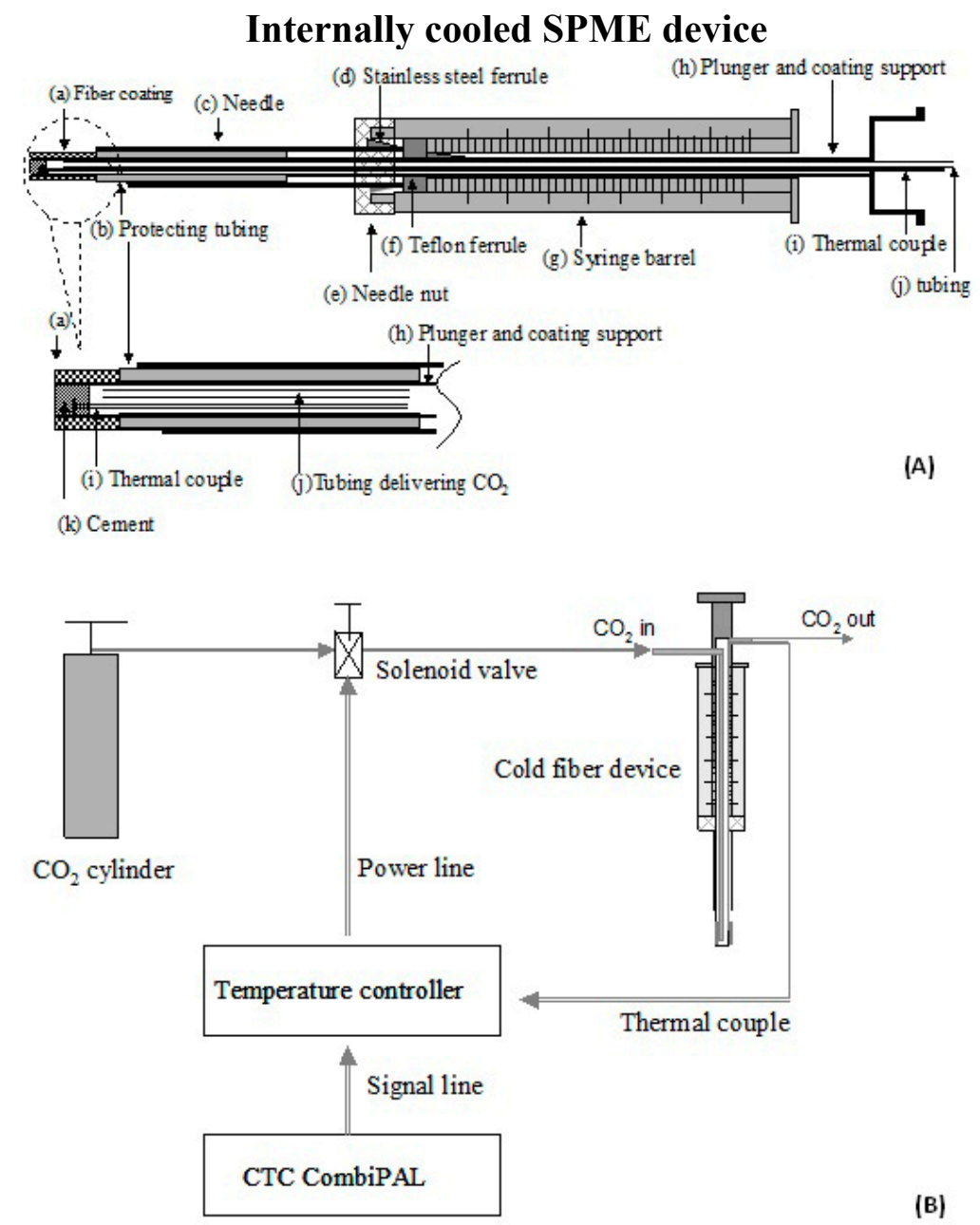

(B)

Figure 2. Internally cooled SPME device (A) and its automation (B) according to Chen and Pawliszyn (2006) [53], reprinted with permission of the American Chemical Society.

\subsection{Non-fiber SPME Techniques}

Non-fiber SPME techniques developed over the last two decades can be divided into static methods using sample stirring and dynamic techniques relying on the flow-through of the sample. The former include stir-bar sorptive extraction (SBSE) and thin-film microextraction (TFME). The latter can be classified as in-needle and in-tip SPME [21,47]. Figure 3 provides an overview of these new techniques. 


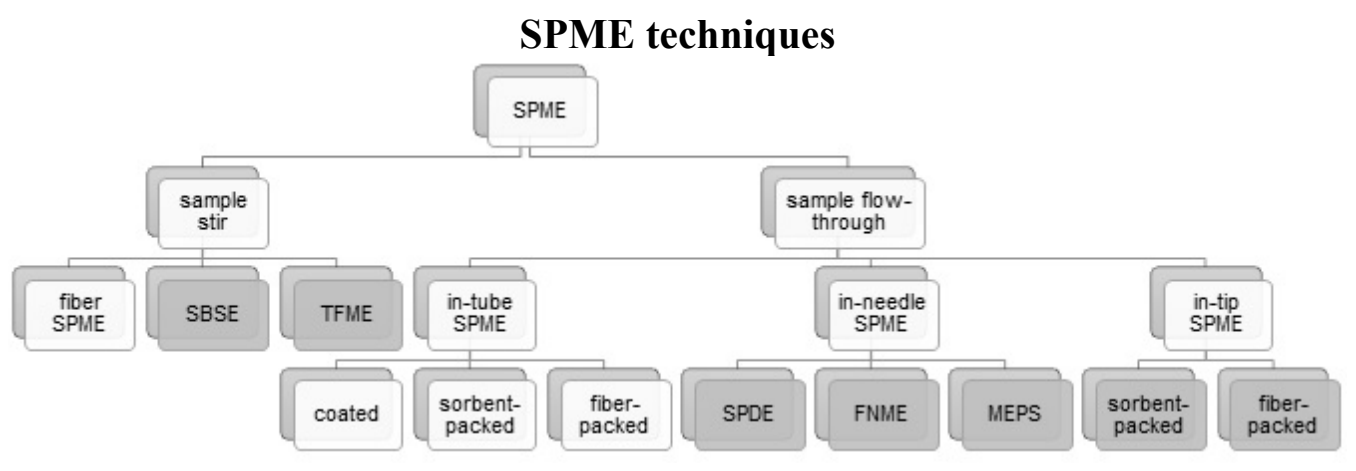

Figure 3. Overview of SPME techniques-techniques marked in grey are more recent developments (SBSE: stir-bar sorptive extraction; TFME: thin-film microextraction; SPDE: solid-phase dynamic extraction; FNME: fiber-packed needle microextraction; MEPS: microextraction by packed syringe).

In stir-bar sorptive extraction (SBSE), a magnetic stir bar coated with polydimethylsiloxane (PDMS) is stirred in or positioned above an aqueous sample [21,50]. After extraction, the stir bar is removed, which is usually done manually with tweezers. In case of a SBSE-GC coupling, desorption of the analytes is induced by inserting the bar into the heated GC injection port or by placing it in a small vial and back-extracting with a few microliters of an appropriate liquid solvent. In case of LC, the mobile phase can be added directly to the stir bar. Compared to fiber SPME, SBSE has a higher concentration capacity as the PDMS and the layer is 50 to 250 times thicker $[21,50]$. However, SBSE is not yet as widely accepted as fiber SPME due to the difficulty of achieving full automation and the limited number of commercially available coatings [21,50]. Dual-phase twisters using different carbon-based adsorbents in addition to PDMS are being developed at the moment as well as other phases covering a wide range of polarities and containing polymers like poly(methacrylic acid stearyl ester-ethylene dimethacrylate) and polypyrrole [21,50].

In thin-film microextraction (TFME), a flat film with a high surface area-to-volume ratio is used as the extraction phase [57,58]. This higher surface-to-volume ratio as well as an increased volume of extraction phase result in an enhanced sensitivity compared to conventional SPME without sacrificing the sampling time.The first step in TFME is the conditioning of the blades. Subsequently, the blades are exposed to the sample to extract the analytes [57,58]. A 96-well plate is placed on an orbital shaker for agitation. After washing with an appropriate solution to remove interfering substances, the blades can be transferred to a second 96-well plate containing the desorption solvent which is eventually injected into the chromatograph. A robotic workstation providing full automation of all the TFME steps including conditioning, extraction, washing and desorption may be used [57,58]. Moreover, coupling to liquid and gas chromatographic systems is possible [57,58]. TFME can be applied to gaseous, liquid and solid samples and is used in different sampling formats. In combination with liquid chromatography, it can be easily performed in a 96-well plate thus allowing for simultaneous analyses. The first TFME application used 96 pieces of small cut SPE disk membranes mounted on a stainless steel wire for support [57,58]. Nowadays, the extraction phase is coated onto a blade-shaped substrate and PDMS is the most common sorbent [57,58]. 
In case of coupling TFME to a gas chromatograph, desorption may also be achieved by direct thin-film injection inside a thermal desorption unit adapted to the special requirements of the thin-film with its particular geometry. The thermal desorption unit contains a large volume inlet and a programmed temperature vaporizer. The latter cryotraps the desorbed compounds. Once the thermal desorption process is completed, the cryotrap is heated and the trapped substances are transferred to the GC column [57,58]. In contrast to solvent desorption, direct thin-film injection allows for a complete introduction of the extracted analytes into the chromatograph $[57,58]$. As a thermal desorption unit is not always available and as solvent-desorption can be carried out in full automation, the latter is often used in practice [57,58].

In-needle SPME methods use a needle instead of a tube for extraction. In 2001, Koziel et al. [59] developed a needle trap (NT) device with quartz wool in order to trap particulate matter and aerosols in air. Wang et al. [60] used this development as a basis for the design of a sorbent-packed NT device for the analysis of VOCs in gaseous samples. The NT device was particularly robust because the quartz wool was protected by the needle. In 2006, Saito et al. [61] developed an in-needle extraction device for the analysis of VOCs using a copolymer of methacrylic acid and ethylene glycol dimethacrylate, which made the procedure more convenient [50,62-64]. This needle showed high extraction performance and thermal stability and was therefore suitable for typical GC applications. Also, the extracted analytes were very stable and could be analyzed even after several days of storage at room temperature [50,62-64].

Today, in-needle SPME methods can be classified as solid-phase dynamic extraction (SPDE), microextraction by packed syringe (MEPS) and fiber-packed needle microextraction (FNME). In SPDE, the analytes derived from a liquid or HS sample are concentrated onto a film of PDMS and 10\% activated carbon coated on the inner wall of a stainless steel needle of a $2.5-\mathrm{mL}$ gas-tight syringe by repeatedly moving the plunger of the syringe up and down [50,62-64]. Recovery of the trapped substances takes place by heat desorption directly into the GC injection port. Full automation is possible [50,62-64]. The first commercially available SPDE devices were developed in 2000 by Chromtech (Idstein, Germany) [50,62-64]. Lipinski [64] was the first who applied SPDE for the analysis of pesticides in water [50,62-64]. A significant advantage of SPDE over fiber SPME is the larger coating volume, which is approximately four to five times the volume of a $100-\mu \mathrm{m}$ fiber and causes an increased in concentration capacity. Moreover, the extraction time is shorter, the repeatability better and the mechanical stability of the SPDE device higher [50,62-64]. On the other hand, carry-over may be an issue in SPDE as the analytes tend to remain on the inner surface of the needle after heat desorption [50,62-64].

Microextraction by packed syringe (MEPS) is a miniaturized form of the classical SPE where sample extraction takes place in a packed bed [50,62-64]. MEPS can be on-line coupled to liquid and gas chromatographic systems and is easily performed in automation. In contrast to SPE, the solid packing material is inserted directly into a needle coupled with a syringe and not into a separate column [50,62-64]. Furthermore, the sample preparation time as well as the volume of the sample and the organic solvents are considerably reduced and the MEPS sorbent can be used up to 100 times [50,62-64]. Sorbent materials include reversed phase $(\mathrm{C} 2, \mathrm{C} 8$ and $\mathrm{C} 18)$, normal phase (silica), restricted access material (RAM) and molecular imprinted polymers (MIPs) [50,62-64]. After the packed bed has been conditioned, the sample solution is pumped several times through the syringe. The sorbent is then washed and the analytes are eluted either with an organic solvent which is injected into the chromatograph afterwards or with the LC mobile phase directly into the chromatographic injector [50,62-64]. Fiber-packed needle 
microextraction (FNME) is an alternative technique using fiber instead of particle materials inside the needle [50,62-64].

In-tip SPME differs from MEPS in that the solid packing material is inserted into pipette tips rather than a needle. As extraction is generally done off-line, only parts of the sample are injected into the chromatograph resulting in a lower sensitivity compared to on-line MEPS [50]. On the other hand, a major benefit of in-tip SPME is that several samples can be handled in parallel. Automation is possible by commercially available systems using 96-well extraction plates and a rosdbot [50]. Sorbents used for in-tip SPME comprise silica and methacrylate monoliths. In contrast to fiber and in-tube SPME, in-tip SPME and MEPS provide quantitative recoveries [50]. On the other hand, these techniques exhibit only a moderate enrichment capacity and they have a tendency towards carry-over [50].

\section{SPME Process}

This section will provide an overview of the different available extraction and desorption techniques for SPME applications. The variety of these techniques is shown in Figure 4. Furthermore it will provide recent information on commercially available fiber coatings as well as current developments in fiber coating procedures. Finally, aspects of quantitation techniques applicable for SPME will be described.

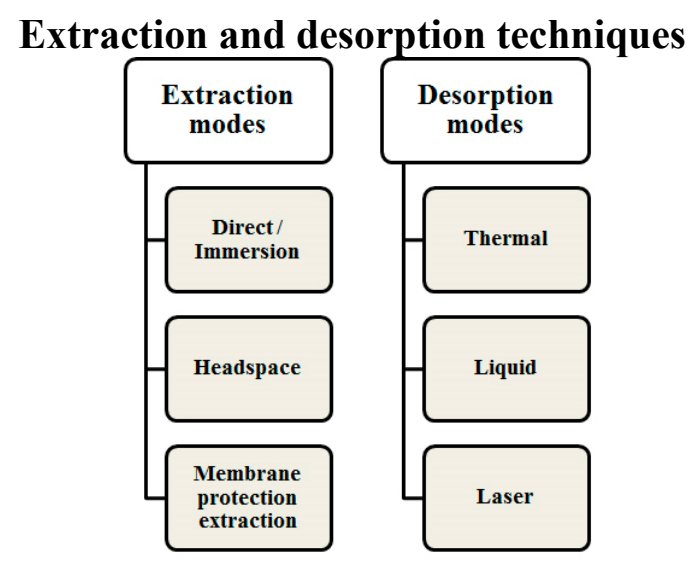

Figure 4. Extraction and desorption techniques for SPME applications.

\subsection{Extraction and Desorption Techniques}

The extraction process of fiber SPME can be conducted in three common ways: direct or immersion extraction (DI), headspace (HS) and membrane protection extraction (see Figures 5 and 6). In Direct SPME the fiber is directly immersed in a gaseous or liquid sample. In case of a liquid sample certain agitation is required to reduce the extraction time. For volatile compounds in gaseous samples, the natural occurring air flow is often sufficient to reach the equilibrium [65].

In HS-SPME the fiber is exposed to the vapor phase above the liquid or solid sample [9]. The advantages are the protection of the fiber from damaging substances of the sample matrix and a possible adjustment of $\mathrm{pH}$ conditions. Only for very volatile compounds a significant difference between DI-SPME and HS-SPME techniques was observed [66]. The extraction kinetics are governed by Henry's law [67]. If the Henry's constant of a given substance is high, then the concentration of the compounds in the headspace is high, too. Under these conditions a rapid extraction from the headspace takes place. 
In case of aqueous sample matrices, volatile and non-polar compounds are extracted faster than semi-volatiles and polar volatiles [65]. Moreover, increasing sample temperature and agitation efficacy may decrease the extraction time [65].

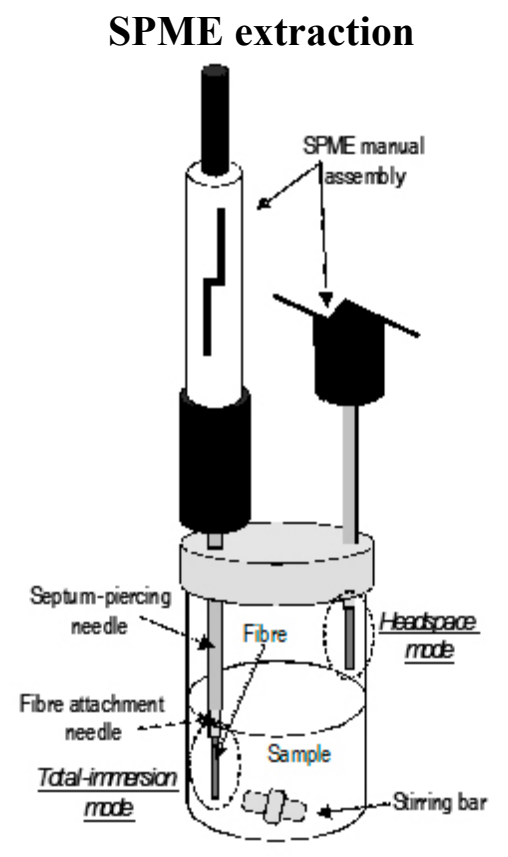

Figure 5. SPME procedure for total-immersion and headspace sampling according to Nerín et al. (2009) [2], reprinted with permission of Springer-Verlag.

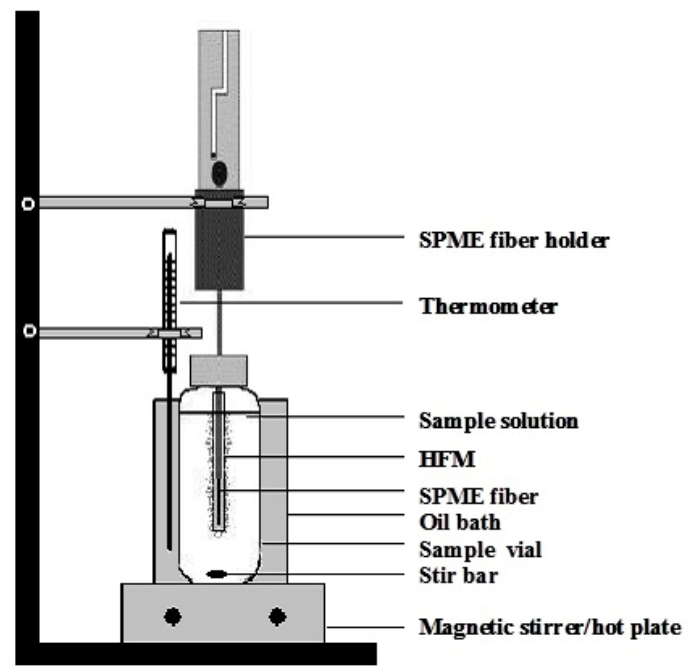

Figure 6. SPME procedure for membrane protection sampling according to Basheer and Lee (2004) [68], reprinted with permission of Elsevier B.V. (HFM: hollow fiber membrane).

In static headspace sampling, diffusion occurs between the fiber and the sample without any interference, whereas dynamic headspace sampling involves air movement devices like air sampling pumps that serve to move the headspace air [69]. To collect and pre-concentrate the headspace gas, the headspace air is transferred into another chamber where the SPME filament or another extraction trap is present [70]. 
An advantage of static headspace sampling in comparison with dynamic headspace sampling is that static headspace sampling does not require careful calibration processes and expensive air sampling pumps [71]. However, differences in temperature and pressure affect the efficiency of the static sampling process as well as wall effects from sampling containers that also play a role [69]. In summary static headspace sampling shows advantages of simplicity, sensitivity, selectivity and ease of automation over dynamic headspace sampling techniques [72].

The third extraction technique is the membrane protection extraction. Extraction which is typically applied to the analysis of samples containing interfering compounds such as proteins, humic acids and fatty material [68]. It is important to prevent the extraction of these high molecular weight compounds which potentially complicate, or even inhibit, sample analysis [68]. The extraction is conducted using a membrane which is selectively permeable for analytes of interest [68]. Membran protection extraction is slow in comparison with other extraction techniques, but effectively extracts compounds with low volatility [65].

All extraction procedures require an optimization of performing parameters in order to achieve good reproducibility, resulting from a compromise between sensitivity and extraction time. Method optimization parameters include agitation conditions, extraction time, extraction technique, temperature, headspace and sample volume vial shape, condition of fiber coating, depth of the SPME fiber inside the vial, geometry of the fiber, and $\mathrm{pH}$ conditions $[33,73]$.

After extraction, target analytes are introduced into a suitable instrument for detection [74]. Desorption can be proceed in two different ways; static mode, by dipping the fiber into the mobile phase or solvent for a specified period, and dynamic mode, where the analytes are desorbed into a flowing mobile phase [65]. It is important to identify desorption solutions that completely remove the analytes from the fiber, eliminating carry over, but that do not damage the sorbent material or degrade the analytes of interest [74].

In the case of a GC coupling the analytes are released by thermal desorption, for which the SPME fiber is inserted into the GC inlet and heated to temperatures that increase analyte volatility sufficient for their release. The analytes are physically released from the sorbent through heating whereby the volatility of the target compounds is increased. The carrier gas flow rate and the injector temperature are critical factors for effective thermal desorption [75]. High desorption temperatures effect rapid transfer of the target analytes from the injector to the chromatographic column, but may reduce the stability of the sorbent and lead to bleeding of the polymeric material [73]. Therefore the thermal stability of the fiber coating determines the upper desorption temperature limit [74].

Combined with an HPLC interface desorption is performed by solvent extraction in the desorption chamber [12], namely liquid desorption. Liquid desorption is conducted using a small volume of suitable solvents to transfer the target analytes to the analytical instrumentation [74]. Desorption of the analytes can also be conducted using a polar organic solvent, such as methanol or acetonitrile [65]. This approach is often used in combination with liquid chromatography (LC) [65]. For thermally labile compounds liquid desorption has the advantage of not requiring elevated temperatures compared to thermal desorption [74]. Liquid desorption is the combination of SPME with HPLC where desorption is achieved using an appropriate extraction solvent in a desorption chamber. Individually designed desorption interfaces have been reported [51,76]. There is a commercially available device that enables desorption of all analytes directly into the LC injector [65]. 
Another form of desorption is conducted by means of laser techniques. SPME in combination with matrix-assisted laser desorption/ionization mass spectrometry was reported [74,77].

\subsection{Derivatization}

Under some conditions target analytes cannot be efficiently extracted or determined by the applied method due to the physicochemical properties of the analytes or the composition of the matrix. In these cases derivatization may modify the analytes and improve their extraction, desorption, or chromatography relative to matrix interferences. Derivatization might also improve the separation performance of analytes with poor chromatographic behavior [78]. Desired requirements for derivatization reactions are a fast and quantitatively formation at room temperature as well as a high stability and solubility in the applied solvent or phase [74].

There are different derivatization methods applicable for SPME (see Figure 7). The main difference of the various derivatisation approaches is the point of time when the derivatization reactions take place. Firstly, the derivatization reaction can be conducted by directly adding the derivatizing agent to the sample, named direct or pre-extraction derivatization. Furthermore, the reaction can take place during the extraction process (simultaneous derivatization and extraction) or immediately after that (derivatization following extraction or post-extraction derivatization). Under these conditions, the derivatization is performed by exposing the enriched SPME fiber to the derivatization agent solution after the extraction [74]. Another option is the post-extraction derivatization, which take place in the injection port of a gas chromatograph through thermal conversion (derivatization in GC Injector Port (see Figure 7).

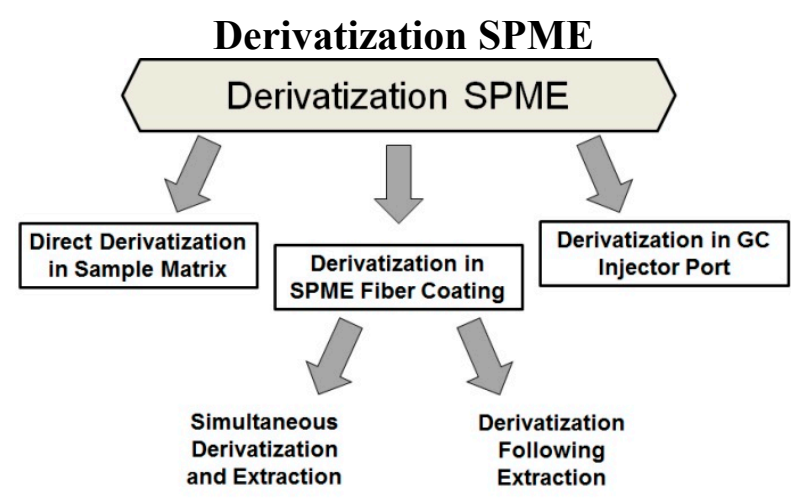

Figure 7. Classification of derivatization techniques in SPME, adapted from Pan and Pawliszyn (1997) [79].

In direct derivatization the chemical structure of the analyte is modified. Afterwards the common extraction and desorption procedures are performed. The obtained derivatives show better performance characteristics than the original analytes by respective extraction phases [79]. Direct derivatization is a simple derivatization technique and is frequently applied and easy to implement [74].

Another option for derivatization takes place simultaneously with the extraction process or after the extraction process. Due to the combination of extraction and derivatization this procedure is quite convenient to apply and the time needed for the analysis can be significantly reduced [74]. Barriers of this procedure are the compatibility of the derivatizing agent with the extraction phase and with the applied extraction method [74]. However, optimization of the composition of the derivatizing agent is 
frequently required [74]. Therefore, post-extraction derivatization is preferred when extraction and derivatization cannot be conducted simultaneously or the partition coefficients of the derivative agents are lower than those of the analytes [74].

Injection port derivatization is a post-extraction derivatization procedure, for which analytes containingpolar functional groups such as $-\mathrm{OH},-\mathrm{SH},-\mathrm{NH}$, and $-\mathrm{COOH}$ are thermally converted into derivatives.

Derivatization in food analysis for example has been applied for analysis of chlorophenols and chloroanisoles in wine [80] or formaldehyde in fish products [81]. Interesting fields of application of derivatization in environmental samples are, for example, the analysis of formaldehyde and other carbonyl compounds in indoor air [82], organometallic compounds (mercury, lead, tin) in river and seawater [83] or organotin compounds in sediment [84]. Further applications of derivatization in the fields of food [80,81,85,86] and environment [82-84,87-96] are provided in Section 3 and 4, respectively. Recently, commonly used derivatization agents were reviewed by Quintana and Rodriguez [97] and Stalikas and Fiamegos [78].

The main drawback of derivatization, however is the formation of artefacts or increased matrix interferences [74]. Furthermore, derivatization reactions favors the generation of waste and therefore, it is recommended to avoid them where possible [98].

\subsection{Salt-addition}

The addition of salting-out reagents, usually sodium chloride or sodium sulphate, increases the ionic strength of the solution. The extraction efficiency is improved by decreasing the solubility of analytes, thus increasing the amount of analyte sorbed on the fiber. However it should be realized that this effect depends on the particular analyte and salt concentration in the sample [99].

\subsection{Fiber Coatings}

The choice of the fiber coating mainly depends on the nature of the analytes. Before a sample is analyzed, the fiber should be cleaned in order to remove contaminants that might increase background noise in the chromatogram [65]. Frequently used commercially available fiber coatings for food and environmental analysis are polydimethylsiloxane (PDMS), polyacylate (PA), Carboxen (CAR; a carbon molecular sieve), divinylbenzene (DVB), and Carbowax (CW; polyethylene glycol). These fibers are available in various coating combinations, blends or copolymers, film thicknesses, and fiber assemblies. The variety is constantly growing increasing the number of applications [11]. The most widespread examples of absorption or liquid fiber coatings are PDMS and PA. Mixed coatings in which the primary extraction phase is a porous solid are also available [100]. CAR/PDMS and DVB/CAR/PDMS fibers provide the best extraction efficiencies for a wide range of analytes with different polarities and molecular weights [65]. PDMS has the ability to withstand high temperatures up to $300{ }^{\circ} \mathrm{C}$ and shows great stability [65]. PDMS fibers are better suited to the analysis of nonpolar analytes, whereas PA fibers are more useful to extract polar analytes [65]. DVB is also a polar porous solid coating and therefore efficient in extracting polar compounds such as disulfides and trisulfides [101]. Bipolar compounds like alcohols, aldehydes, ketones, ethers and carboxylic acids are often most efficiently extracted by fibers consisting of a combination of e.g., non-polar material (PDMS) and a polar material (DVB) [65]. 
Another appropriate choice to extract analytes with different polarities are coating mixtures consisting of CARB/PDMS or DVB/CARB/PDMS [65]. However, a disadvantage of this coating material is the displacement effect of analytes with a lower affinity to the coating [102]. Apart from commerciallyavailable sorbents, different new coating procedures have been applied to expand coating types in the commercially applicable and reproducible SPME devices. These new approaches also address biocompatibility, on-site compatibility, selectivity and sensitivity limitations [103,104]. One can differentiate coating procedures based on the sorbent type into dipping and physical agglutinating methods, sol-gel technology, chemical grafting, electrochemical methods, electrospinning, liquid-phase deposition and the hydrothermal method [24]. Further new coatings are polypyrrole polymer coatings [105], molecularly imprinted polymers (MIPs) [106] and immunoaffinity systems [107].

The following section gives a brief overview of the variety of coating procedures. More detailed information, including advantages and disadvantages of various coatings, is available in other recent technology reviews $[11,24]$.

Table 1 lists the different coating procedures, sorbent materials and examples of applications.

Physical coating processes were the first to be developed and constitute the most convenient procedures [11]. They are compatible with nearly all kinds of sorbent materials. Application of new coatings such as carbon nanomaterials (CNMs), ordered mesoporous materials, ionic liquids (ILs) and polymeric ionic liquids (PILs) is very simple. These coatings can be used for fused silica fibers as well as for metal wires. The Dipping process is conducted by placing a fiber in a concentrated organic solvent solution of the material to be deposited for a short time. Crosslinking of the deposited material can take place after evaporation of the solvent by drying. In case of a metal wire, support material can be attached using a sticky paste layer of the sorbent (epoxy or similar) $[11,24]$.

An alternative to physical coating procedures is the sol-gel technology developed by Chong and co-workers (1997) [108]. Sol-gel process is based on the building of an inorganic network consisting of a colloidal suspension (sol) and gelation of this sol which forms a network in a continuous liquid phase (gel). The extraction phase can be chemically bonded to the silica thus producing highly cross-linked phase networks [2]. The precursors for the synthesis of the colloids consist of a metal or metalloid element (alkoxysilanes tetramethoxysilane or tetraethoxysilane (TMOS/TEOS) surrounded by various reactive ligands [109]. The sol-gel fibers therefore exhibit higher thermal stability, hydrolytic stability towards organic solvents as well as high and low $\mathrm{pH}$ solutions, and higher surface areas. Molecularly selective coatings can be prepared by using substances with specific functionalities such as crown ethers or B-cyclodextrin derivatives [2]. Kumar et al. (2008) published a review on the development of sol-gel methods including various applications (e.g., PAHs, aromatic amines, phenols and pesticides) in environmental, pharmaceutical and food analysis [2,25]. A large number of new extraction phases with unique features have been introduced by application of the sol-gel technology. The most important advancements are summarized in Table 1. As there is no standardized sol-gel procedure, this new technology lacks inter-laboratory reproducibility [11]. Further disadvantages of the sol-gel technology are the large number of optimization factors and the fragility of the fused silica fiber used mostly as a support. Due to these limitations of the sol-gel procedures, simple chemical grafting has been developed. In this technique, the surface of the sorbents and supports are chemically modified in order to allow them to react with each other. Possible sorbents applicable to both metal wires and fused silica fibers are nanomaterials, antibodies, MIPs, ILs and PILs [24]. 
Electrochemical procedures are able to generate extraction films of variable thickness on an unbreakable metal support at a low cost and with a simple setup. Coatings show high thermal stability, have a long shelf life and some may provide higher capability to sorb polar compounds. Electrochemical procedures may be divided into electrodeposition, anodization and electrophoretic deposition (EPD) [110]. Electrodeposition can be used to create coatings with porous structure and refers to the deposition of a metallic or conductive polymer (CP) coating onto a base material by the electrochemical reduction of metal ions or electropolymerization of CPs from an electrolyte. This technique has been applied to the coating of metal supports with CPs, CPs nanocomposites, PIL composites and metal oxides [24]. Anodized metal wires were first developed by Djozan and co-workers in 2001 [111]. They anodized aluminum wires by direct current in sulfuric acid to obtain a porous layer of aluminum oxide on the aluminum surface [110]. Electrophoretic deposition (EPD) is a direct particle assembly method that is based on particle coagulation rather than an electrochemical reaction. Charged nanoparticles from a solution are deposited onto a substrate using an electric field [24]. Single-walled carbon nanotube (SWCNT) [112-114] and multi-walled carbon nanotube (MWCNT) [115] coatings have been prepared on the surfaces of platinum and stainless steel wires, respectively. In EPD, adsorption takes place in the spaces between the nanotubes and amorphous carbon in a three-dimensional network of nanotubes [24].

In electrospinning, a solution of a high molecular weight polymer with high viscosity is drawn into nanofibers by repulsive electrostatic forces. Zewe et al. (2010) were the first to prepare nanostructured polymeric based SPME coatings by electrospinning. They used a polymeric negative photoresist, SU-8 2100, which was converted to carbon by pyrolysis. The resultant SPME devices were used to extract both nonpolar and polar compounds [116].

Lin et al. (2008) employed liquid phase deposition (LPD) to produce a nanomaterial-based in-tube SPME coating with increased extraction efficiency [117]. Due to the chemical bonding between the substrate and the coating, LPD offers highly stable nanomaterial thin film coatings. It has been used to deposit thin films of $\mathrm{SiO}_{2}, \mathrm{TiO}_{2}, \mathrm{SnO}_{2}, \mathrm{ZrO}_{2}$ and the three dimensional transition metal oxides $\mathrm{V}, \mathrm{Cr}$, $\mathrm{Mn}, \mathrm{Fe}, \mathrm{Co}, \mathrm{Ni}, \mathrm{Cu}, \mathrm{Zn}$, In (individually or combined) [24].

The hydrothermal growth technique has been used to produce $\mathrm{ZnO}$ nanoparticles on the surface of fused silica fiber and stainless steel wire [118,119]. At first, the procedure consisted of two steps, seeding and growing [24]. A simplification to a single step was realized by Alizadeh et al. (2011) [118]. It is possible to coat 50 fibers at once. The hydrothermal method is used to produce organic framework (MOF) SPME coatings which offer high thermal and mechanical stability and exceptionally large surface areas [24].

In their review on procedures for the preparation of SPME coatings, Aziz-Zanjani and Mehdinia (2014) conclude that this variety of coatings forms a good basis for the development of more designable structures. Durable and stable SPME devices can be produced by metal supports and chemical bonding between support and sorbent. However, poor selectivity and limited applicability to samples with complex matrices remain problematic [24]. 
Table 1. Coating procedures and sorbent materials used [24].

\begin{tabular}{|c|c|c|c|c|c|c|}
\hline $\begin{array}{l}\text { Dipping and } \\
\text { physical } \\
\text { agglutinating } \\
\text { methods } \\
\end{array}$ & Sol-gel technology & Chemical grafting & Electrochemical methods & $\begin{array}{l}\text { Electro- } \\
\text { spinning }\end{array}$ & $\begin{array}{l}\text { Liquid-phase } \\
\text { deposition }\end{array}$ & $\begin{array}{l}\text { Hydro-thermal } \\
\text { methods }\end{array}$ \\
\hline $\begin{array}{l}\text { Carbon } \\
\text { nanomaterials } \\
{[26,120]}\end{array}$ & $\begin{array}{l}\text { Functionalized or polymer- } \\
\text { functionalized carbon } \\
\text { nanomaterials } \\
{[125,126,127]}\end{array}$ & $\begin{array}{l}\text { Nanomaterials }[138,139] \\
\text { Immunoaffinity SPME } \\
{[140]}\end{array}$ & $\begin{array}{l}\text { Electrodeposition } \\
\text { Electropolymerized } \\
\text { conductive polymers } \\
{[88,145-147]}\end{array}$ & $\begin{array}{l}\text { Electrospun } \\
\text { epoxide } \\
\text { polymer }\end{array}$ & $\begin{array}{l}\mathrm{SiO}_{2}, \mathrm{TiO}_{2}, \\
\mathrm{SnO}_{2}, \mathrm{ZrO} 2 \\
{[117]}\end{array}$ & $\begin{array}{l}\mathrm{ZnO} \\
\text { nanoparticles } \\
\text { Organic }\end{array}$ \\
\hline $\begin{array}{l}\text { Ordered } \\
\text { mesoporous } \\
\text { materials } \\
{[121,122]}\end{array}$ & $\begin{array}{l}\text { Ionic liquid-mediated } \\
\text { SPME coating }[128,129]\end{array}$ & $\begin{array}{l}\text { Molecularly imprinted } \\
\text { polymers }[141,142]\end{array}$ & $\begin{array}{l}\text { Electropolymerized } \\
\text { conductive polymers } \\
\text { nanocomposite }[148,149]\end{array}$ & $\begin{array}{l}\text { Carbon } \\
\text { nanofiber- } \\
\text { based SPME } \\
{[116,156,157]}\end{array}$ & $\begin{array}{l}\text { Three } \\
\text { dimensional } \\
\text { transition } \\
\text { metal oxides }\end{array}$ & $\begin{array}{l}\text { frameworks } \\
\text { (MOFs) } \\
{[118,119]}\end{array}$ \\
\hline \multirow{5}{*}{$\begin{array}{l}\text { Ionic liquids and } \\
\text { polymeric ionic } \\
\text { liquids } \\
{[123,124]}\end{array}$} & $\begin{array}{l}\text { Sol-gel derived polymeric } \\
\text { ionic liquid-based SPME } \\
\text { coatings }[130,131]\end{array}$ & $\begin{array}{l}\text { Substrate-bonded ionic } \\
\text { liquid coatings [143] }\end{array}$ & $\begin{array}{l}\text { Conductive polymer-ionic } \\
\text { liquid composites }[150,151]\end{array}$ & & $\begin{array}{l}(\mathrm{V}, \mathrm{Cr}, \mathrm{Mn}, \\
\mathrm{Fe}, \mathrm{Co}, \mathrm{Ni}, \mathrm{Cu}, \\
\mathrm{Zn}, \mathrm{In},\end{array}$ & \\
\hline & $\begin{array}{l}\text { Sol-gel ordered } \\
\text { mesoporous silica SPME } \\
\text { coating }[132,133]\end{array}$ & $\begin{array}{l}\text { Substrate-bonded } \\
\text { polymeric ionic liquid } \\
\text { coatings [144] }\end{array}$ & $\begin{array}{l}\text { Metal oxides }[152,153] \\
\frac{\text { Anodized metal wires }}{\text { Metal oxides }[154,155]}\end{array}$ & & $\begin{array}{l}\text { individually or } \\
\text { combined) } \\
{[158]}\end{array}$ & \\
\hline & $\begin{array}{l}\text { Sol-gel coating on metal } \\
\text { wires }[134,135]\end{array}$ & & $\frac{\text { Electrophoretic }}{\text { deposition }}$ & & & \\
\hline & $\begin{array}{l}\text { Sol-gel molecularly } \\
\text { imprinted polymer coatings } \\
{[113,136]}\end{array}$ & & $\begin{array}{l}\text { Carbon nanotubes (CNTs) } \\
{[112-115]}\end{array}$ & & & \\
\hline & $\begin{array}{l}\text { Aptamer Sol-gel SPME } \\
\text { [137] }\end{array}$ & & & & & \\
\hline
\end{tabular}




\subsection{Quantitation}

Contrary to traditional sample preparation methods such as liquid-liquid extraction, solid-phase extraction or Soxleth, SPME is a non-exhaustive technique where only a fraction of the target analyte is extracted from the sample [22]. Consequently SPME must be calibrated carefully for quantitative analysis.

Quantitation of analytes in complex food and environmental matrices is currently one of the major challenges of SPME procedures. Nevertheless, several international standards applying the SPME methodology have recently been implemented, proving that SPME has been accepted as a state-of-the-art analytical technique at least in some fields of application (e.g., environmental). Firstly, the International Standard ISO 27108 [159] "Water quality-Determination of selected plant treatment agents and biocide products-Method using solid-phase microextraction (SPME) followed by gas chromatography-mass spectrometry (GC-MS)" has been established. The described procedure is based on the German Standard DIN 38407-34 [160]. Another standard based on DIN 38407-41 [161] has been published as a Draft International Standard in 2014: "Water quality - Determination of volatile organic compounds in waterMethod using headspace solid-phase microextraction (HS-SPME) followed by gas chromatographymass spectrometry (GC-MS) (ISO/DIS 17943) [162]. In addition, two other standards applying SPME methods have been published: the Austrian OENORM A 1117 [163] (Determination of volatile compounds in cellulose based materials by SPME) and the American ASTM D 7363a [164] (Standard test method for determination of parent and alkyl polycyclic aromatics in sediment pore water using solid-phase microextraction and gas chromatography/mass spectrometry in selected ion monitoring mode).

Essential to developing quantitative SPME methods is the knowledge of fundamental principles governing the mass transfer of analytes in multiphase systems, such as thermodynamics and mass-transfer kinetics $[5,43,44]$. The current available SPME calibration methods can be categorized into traditional calibration methods including external standard, internal standard and standard addition as well as newer methods such as equilibrium extraction, exhaustive extraction and diffusion-based calibration (see Table 2). Ouyang and Pawliszyn [18,22] published a review giving more detailed information about the mentioned individual methods.

\subsubsection{Traditional Calibration Methods}

Traditional calibration methods include external standards (calibration curve), internal standard or standard addition method.

SPME calibration can be performed either by equilibrium or pre-equilibrium calibration, respectively. In equilibrium calibration a partitioning equilibrium between the sample matrix and extraction phase is reached. The convection conditions do not affect the amount of extracted analytes due to the present partitioning equilibrium [22]. In pre-equilibrium calibration the amount of extracted analytes is related to the extraction time [22]. The convection/agitation thereby is constant. Applying equilibrium calibration for quantitation purpose is more sensitive and does not depend on the time. However, if the equilibrium extraction is too long, the pre-equilibrium extraction is preferred [22].

The internal standard method is widely applied for quantitation in SPME methods. It has been applied for the quantitative analysis of environmental $[165,166]$ and food $[167,168]$ samples. For heterogeneous samples the standard addition calibration method is preferred $[169,170]$. Using an internal or a surrogate standard (preferably mass-labeled internal standards) compensates for matrix effects and instrumental or sample preparation variability [171-175]. In addition, external standards are widely used [22,176-180]. 
Table 2. SPME calibration methods [22].

\begin{tabular}{|c|c|c|c|c|}
\hline Calibration method & & Advantages & Disadvantages & Applications \\
\hline \multirow[t]{5}{*}{ Traditional } & \multirow[t]{2}{*}{ External standard } & \multirow[t]{3}{*}{ No extensive sample preparation } & $\begin{array}{l}\text { Need for availability of blank sample } \\
\text { matrices }\end{array}$ & {$[176-178]$} \\
\hline & & & $\begin{array}{l}\text { Need for stable sampling procedure } \\
\text { and chromatographic conditions }\end{array}$ & {$[169,170]$} \\
\hline & Standard addition & & $\begin{array}{l}\text { Extensive sample preparation and } \\
\text { analysis }\end{array}$ & {$[165-168,171,172]$} \\
\hline & \multirow[t]{2}{*}{ Internal standard } & Correction of sample matrix effects & $\begin{array}{l}\text { Limited availability of suitable internal } \\
\text { standards }\end{array}$ & \\
\hline & & $\begin{array}{l}\text { Compensation of matrix effects and } \\
\text { losses of analytes during sample } \\
\text { preparation and irreproducibility in } \\
\text { parameters (injection in GC/LC) }\end{array}$ & $\begin{array}{l}\text { High cost and limited availability of } \\
\text { isotope-labelled standards }\end{array}$ & \\
\hline $\begin{array}{l}\text { Equilibrium } \\
\text { extraction }\end{array}$ & & $\begin{array}{l}\text { Possibility to calculate concentration of } \\
\text { analytes by amount of extracted } \\
\text { analytes } \\
\text { Independence of amount of extracted } \\
\text { analytes of sample volume }\end{array}$ & $\begin{array}{l}\text { Need for knowledge about distribution } \\
\text { coefficients }\end{array}$ & {$[181,182]$} \\
\hline $\begin{array}{l}\text { Exhaustive } \\
\text { extraction }\end{array}$ & & $\begin{array}{l}\text { Possibility to calculate concentration of } \\
\text { analytes by amound of extracted } \\
\text { analytes and sample volume }\end{array}$ & $\begin{array}{l}\text { Suitable only for small sample } \\
\text { volumes and large distribution } \\
\text { coefficients or need for special devices }\end{array}$ & {$[56,183]$} \\
\hline
\end{tabular}


Table 2. Cont

\begin{tabular}{|c|c|c|c|c|}
\hline Calibration method & & Advantages & Disadvantages & Applications \\
\hline \multirow[t]{4}{*}{ Diffusion-based } & $\begin{array}{l}\text { Fick's first law of } \\
\text { diffusion }\end{array}$ & $\begin{array}{l}\text { Suitability for TWA sampling } \\
\text { Independency of sampling rate of face } \\
\text { velocity }\end{array}$ & $\begin{array}{l}\text { Sorbent should be zero sink for target } \\
\text { analytes } \\
\text { Very low sample rate for water } \\
\text { sampling }\end{array}$ & {$[184,185]$} \\
\hline & $\begin{array}{l}\text { Interface model } \\
\text { and cross-flow } \\
\text { mode }\end{array}$ & $\begin{array}{l}\text { Minimizing of competitive effects for } \\
\text { solid coating through high sampling } \\
\text { rate and short time } \\
\text { Suitability for on-site sampling }\end{array}$ & $\begin{array}{l}\text { Need for controlled or determined flow } \\
\text { velocity of sampling matrix } \\
\text { Application limited to linear sampling } \\
\text { regime }\end{array}$ & {$[186,187]$} \\
\hline & $\begin{array}{l}\text { Kinetic calibration } \\
\text { with standard }\end{array}$ & Suitability for TWA sampling & $\begin{array}{l}\text { Need for determination of standard } \\
\text { loading }\end{array}$ & {$[188,189]$} \\
\hline & $\begin{array}{l}\text { Standard-free } \\
\text { kinetic calibration }\end{array}$ & $\begin{array}{l}\text { No need for standard loading } \\
\text { Possibility to calculate concentrations } \\
\text { of all extracted analytes in sample }\end{array}$ & $\begin{array}{l}\text { Need for stable sampling conditions } \\
\text { Unsuitability for long-term monitoring }\end{array}$ & [190] \\
\hline
\end{tabular}




\subsubsection{Equilibrium Extraction}

The equilibrium extraction method is a widely applied method for quantitation [181,182]. A small part of the extraction phase is exposed to the sample until equilibrium is reached. The concentration of the extracted analyte in the extraction phase is not dependent on the sample volume but corresponds directly to its concentration in the sample [22]. Therefore in practice, there is no need to collect a defined sample prior to analysis. The fiber can be exposed directly to the sample. The analytical process can be accelerated by removing the sampling step. Errors related to analyte losses through the decomposition or adsorption on the sampling container walls will be prevented. In that case the concentration of the target analytes can be determined. However, the distribution coefficients of the analytes between the fiber coating and the sample matrix have to be determined by experimentations. The concentration then can be determined by the amount of the analytes on the fiber under extraction equilibrium.

This calibration method was successfully applied for on-site air [181] or water sampling [182]. For air sampling the extraction can be performed in static and dynamic model [22]. In dynamic model, extraction rates from air-samples can be increased significantly with an air pump [181].

\subsubsection{Exhaustive Extraction}

Exhaustive extraction calibration can be done by using an internally cooled fiber device [52]. The higher analyte recoveries achieved using internally cooled fiber extraction result in greater confidence in quantitation and reporting of analyte concentrations (see Section 2.3, Figure 2). Almost quantitative extraction of the analytes was reported [22].

\subsubsection{Diffusion-based Calibration}

The diffusion coefficient is essential for description of kinetic processes of SPME [22]. Recently various diffusion-based calibration methods have been developed [22]. They were developed based on Fick's first law of diffusion, the interface model, the cross-flow model and the kinetic process of absorption/adsorption and desorption [22]. Main applications are in on-site sampling, comprising grab sampling and long-term monitoring [22].

Fick's first law is applicable to calibration when diffusion paths are well-defined [22]. The main applications are air and water sampling. Fiber-retracted SPME devices are used in which the analyte molecules access the fiber coating only by means of diffusion through the static air/water gap between the needle opening and the fiber coating. In air, the diffusion of the molecules is fast and the length of the diffusion path can be adjusted [191]. These kinds of samplers were applied for time-weighted average (TWA) sampling of several analytes in air [56,184]. For water sampling, a commercially SPME fiber assembly was developed and used for monitoring polycyclic aromatic hydrocarbons (PAHs) in water [185].

In the case of a poorly defined or unknown diffusion path for on-site sampling (when the SPME fiber is inserted directly into the air or water sample , the interface model and cross-flow model can be used for calibration. However, these models are limited to the linear sampling regime and a constant convection of air/water [22]. Applying these calibration methods requires a controlled velocity of air/water [22]. The Interface model was developed by Koziel et al. [186] and applied for sampling of volatile organic compounds (VOC) in air. The cross-flow model was developed by Chen et al. [187]. 
Kinetic calibration is based on a diffusion-controlled mass transfer model proposed by Ai [43,44]. The underlying assumption of this dynamic model is that there is a linearly proportional relationship between the adsorbed analyte and its initial concentration in the sample matrix. Based on this model two calibration methods of SPME, the kinetic calibration with standard or in-fiber standardization technique [192] and the standard-free kinetic calibration [190] were proposed. The applicability of this technique for TWA water sampling was established by both theoretical derivations and field trials $[188,189]$. Furthermore this method has become more convenient and applicable through the single point calibration [193]. Kinetic calibration with standards in the extraction phase can be applied to grab sampling as well as long-term monitoring [22]. Due to the loss of the standard during sampling this calibration method may not work in some fast sampling situations. For fast on-site and in vivo analysis, a standard-free kinetic calibration method was developed [190]. With this calibration method, all analyes can be directly calibrated with only two samplings and can be quantified without bearing in mind of reaching equilibrium in the system. The method was validated for identification of PAHs and BTEX in a standard aqueous solution and a standard gas flow-through system [190]. Compared with the previous calibration methods for rapid on-site analysis by SPME this method does not require a standard to calibrate the extraction. The total amount of the extracted analytes can be quantified without bearing in mind of reaching equilibrium in the system [190].

\section{Applications in Food Analysis}

The research of aroma-active compounds in foods was developed simultaneously with the introduction of the first gas chromatographs in the early 1960s [194]. In the headspace of foodstuffs the occurrence of around 10,000 compounds was estimated [195]. When considering the choice of suitable methods for food analysis, it is essential to realize that food is a complex, heterogeneous mixture composed of a multitude of different compounds. The analysis of volatile target analytes requires the extraction from the food matrix. It is nearly impossible to analyse food samples without sample pretreatment even though advanced techniques of separation and identification have been made available. Therefore, sample extraction, removal of interferences and the pre-concentration of analytes are mandatory procedures [196].

Developing reliable and sensitive SPME methods for food analysis requires the selection and optimization of parameters like the fiber coating, sampling technique, agitation conditions, sample volume and extraction and desorption conditions [197]. Simultaneous variation of several control variables is possible by applying multivariate test designs reducing the experimental effort for SPME optimization [198]. Multivariate test designs such as full factorial design, Doehlert matrix, $2^{4}$ experimental design and Design of Experiments are valid multivariate test designs for SPME method optimization [55,56,199].

SPME has been widely applied to the sampling and analysis of food matrices. Table 3 provides a comprehensive overview of SPME applications divided into different analyte classes; aroma, off-flavor (food products), off-Flavor (food packaging migrants) and volatile toxic compounds. Figure 8 shows the numbers of papers dealing with applications of SPME to the different types of analytes based on a ScienceDirect literature research for years 2005 to 2015. Hundreds of papers reporting SPME applications in the field of food analysis have been published in recent years (see Table 3). The vast majority of 
which referred to aroma analysis (see Figure 8). The applications of SPME to different food matrices are shown in Figure 9. The majority of applications refer to the food classes fruits/vegetables, fats/oils, wine, meat, dairy and (non-) alcoholic-beverages. The results are comparable to those published by Jeleń et al. [13] derived from a Web of Knowledge search in food aroma analysis between 2006 and 2011.

The microextraction technology in greatest use second to SPME (91\%) was SBSE (5\%), followed by SDME and LLME. The advantage of SBSE is a particularly high sensitivity for semi volatiles. However, SBSE suffered for a long time due to only one available coating (PDMS). Due to the lack of automated versions of SDME and LLME, and also due to their lower popularity in the scientific community (compared to SPME and SBSE) their use in aroma analysis is yet not enough explored. The diversity of matrices for which microextraction methods other than SPME are used proves that these methods await broader applications in aroma analysis [13].

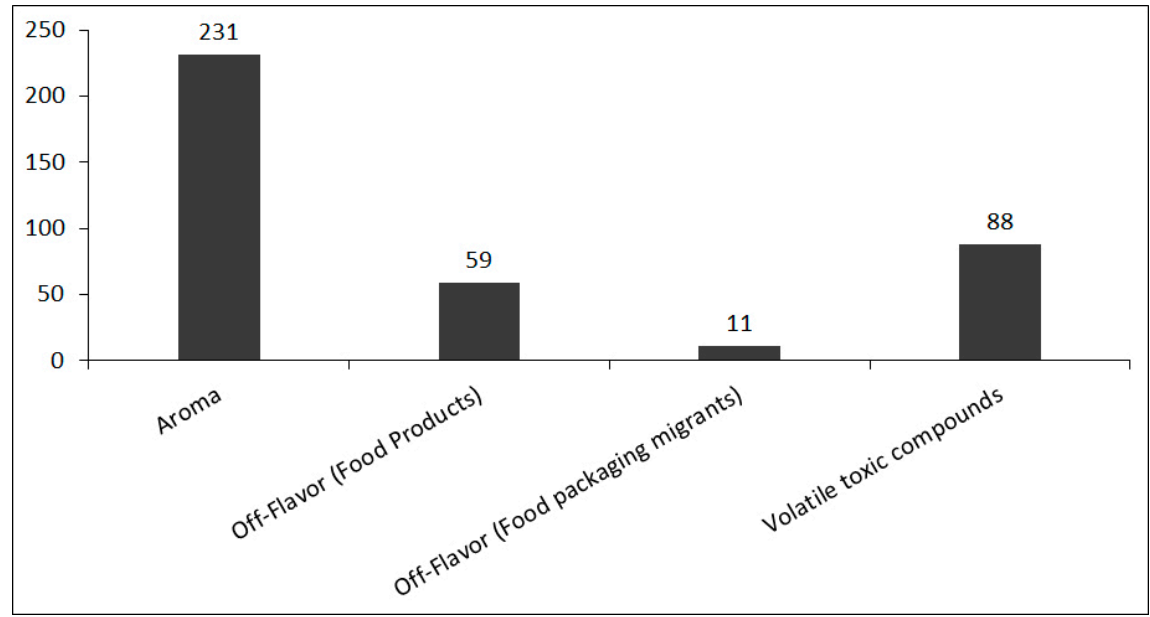

Figure 8. Application of SPME to different types of analytes. Number of papers based on ScienceDirect search for years 2005-2015.

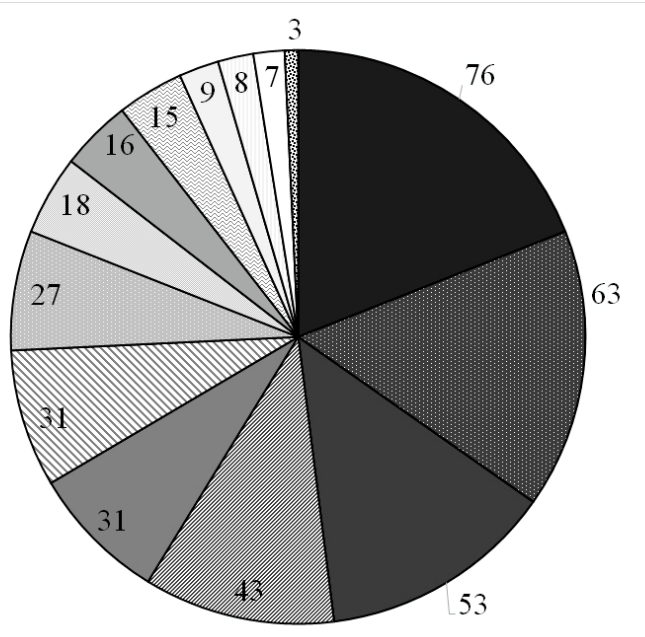

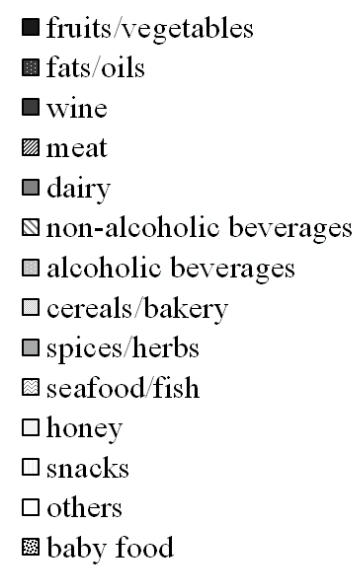

Figure 9. Application of SPME to different food matrices. Number of papers based on ScienceDirect search for years 2005-2015. 
Table 3. SPME applications in food analysis.

\begin{tabular}{|c|c|c|c|c|c|c|c|c|c|c|c|c|c|}
\hline & & & Extraction & nditior & & & & $\begin{array}{l}\text { Deso } \\
\text { conc }\end{array}$ & $\begin{array}{l}\text { rption } \\
\text { ditions }\end{array}$ & & & & \\
\hline $\begin{array}{l}\text { Food } \\
\text { Sample }\end{array}$ & Analyte & Technique & Fiber & $\begin{array}{l}\text { Time } \\
\text { [min] }\end{array}$ & $\begin{array}{l}\text { Temp. } \\
{\left[{ }^{\circ} \mathrm{C}\right]}\end{array}$ & $\begin{array}{l}\text { Salt } \\
\text { addition }\end{array}$ & Deriv. & $\begin{array}{l}\text { Time } \\
\text { [min] }\end{array}$ & $\begin{array}{l}\text { Temp. } \\
{\left[{ }^{\circ} \mathrm{C}\right]}\end{array}$ & Detection & LOD & Recovery & Ref. \\
\hline \multicolumn{14}{|c|}{ Aroma } \\
\hline \multicolumn{14}{|c|}{ Dairy products } \\
\hline Butter & diacetyl & - & PDMS-DVB & 5 & 37 & - & - & 1 & 250 & $\begin{array}{l}\text { GC-MS- } \\
\text { MS }\end{array}$ & $0.0078 \mathrm{ppm}$ & - & [200] \\
\hline $\begin{array}{l}\text { European } \\
\text { PDO hard } \\
\text { cheeses }\end{array}$ & VVC & HS-SPME & DVB-CAR-PDMS & 60 & 45 & - & - & 10 & 260 & $\begin{array}{l}\text { GC-MS / } \\
\text { GC-FID }\end{array}$ & - & - & [201] \\
\hline $\begin{array}{l}\text { PDO } \\
\text { Cheese, } \\
\text { Oscypek }\end{array}$ & VVC & HS-SPME & CAR-PDMS & 15 & 50 & - & - & 5 & 260 & GC-MS & - & - & [202] \\
\hline $\begin{array}{l}\text { Van Herby } \\
\text { Cheeses }\end{array}$ & $\begin{array}{l}\text { esters, ketones, alde- } \\
\text { hydes, acids, alcohols, } \\
\text { hydrocarbons, } \\
\text { terpenes }\end{array}$ & HS-SPME & DVB-CAR-PDMS & 30 & 40 & - & - & 5 & 250 & $\begin{array}{l}\text { GC-MS / } \\
\text { GC-FID }\end{array}$ & - & - & [203] \\
\hline $\begin{array}{l}\text { Milk, } \\
\text { cheese and } \\
\text { whey } \\
\text { powder }\end{array}$ & VVC & HS-SPME & DVB-CAR-PDMS & 30 & 40 & $4 \mathrm{~g} \mathrm{NaCl}$ & - & 5 & 250 & GC-MS & - & - & [204] \\
\hline \multicolumn{14}{|c|}{ Meat and meat products } \\
\hline $\begin{array}{l}\text { Roasted } \\
\text { pork of } \\
\text { mini-pig }\end{array}$ & VVC & DI-SPME & CAR-PDMS & 60 & 80 & $0.22 \mathrm{~g}$ salt & - & 4 & 280 & $\begin{array}{l}\text { GC-MS / } \\
\text { GO-O / } \\
\text { GC-FID }\end{array}$ & - & - & [205] \\
\hline
\end{tabular}


Table 3. Cont

\begin{tabular}{|c|c|c|c|c|c|c|c|c|c|c|c|c|c|}
\hline \multirow[b]{2}{*}{$\begin{array}{l}\text { Food } \\
\text { Sample }\end{array}$} & \multirow[b]{2}{*}{ Analyte } & \multicolumn{6}{|c|}{ Extraction conditions } & \multicolumn{3}{|c|}{$\begin{array}{l}\text { Desorption } \\
\text { conditions }\end{array}$} & \multirow[b]{2}{*}{ LOD } & \multirow[b]{2}{*}{ Recovery } & \multirow[b]{2}{*}{ Ref. } \\
\hline & & Technique & Fiber & $\begin{array}{l}\text { Time } \\
{[\mathrm{min}]}\end{array}$ & $\begin{array}{l}\text { Temp. } \\
{\left[{ }^{\circ} \mathrm{C}\right]}\end{array}$ & $\begin{array}{l}\text { Salt } \\
\text { addition }\end{array}$ & Deriv. & $\begin{array}{l}\text { Time } \\
{[\mathrm{min}]}\end{array}$ & $\begin{array}{l}\text { Temp. } \\
{\left[{ }^{\circ} \mathrm{C}\right]}\end{array}$ & Detection & & & \\
\hline \multicolumn{14}{|c|}{ Meat and meat products } \\
\hline $\begin{array}{l}\text { Traditional } \\
\text { smoke- } \\
\text { cured bacon } \\
\text { (CSCB) }\end{array}$ & $\begin{array}{l}\text { alkane, aldehydes, } \\
\text { ketones, alcohols, } \\
\text { thioethers, thiols, } \\
\text { furans, phenols }\end{array}$ & - & $\begin{array}{l}\text { CAR-PDMS / DVB- } \\
\text { CAR-PDMS }\end{array}$ & 30 & 60 & - & - & 5 & 280 & GC-MS & - & - & [206] \\
\hline $\begin{array}{l}\text { Minced } \\
\text { beef }\end{array}$ & VVC & HS-SPME & DVB-CAR-PDMS & 30 & 40 & $25 \% \mathrm{NaCl}$ & - & 1 & 250 & GC-MS & - & - & [207] \\
\hline $\begin{array}{l}\text { Cooked, } \\
\text { cured pork } \\
\text { ham }\end{array}$ & VVC & $\begin{array}{l}\text { HS-SPME } \\
\text { / SBSE }\end{array}$ & $\begin{array}{l}\text { CAR-PDB-DVB / } \\
0.5 \mathrm{~mm} \text { PDMS phase } \\
\text { thickness stir bars }\end{array}$ & $30 / 90$ & $\begin{array}{l}40 / \\
\mathrm{RT}\end{array}$ & - & - & 10 & $\begin{array}{l}250 / \\
30\end{array}$ & GC-MS & - & - & [208] \\
\hline $\begin{array}{l}\text { Slow } \\
\text { fermented } \\
\text { sausages }\end{array}$ & $\begin{array}{l}\text { dimethyl trisulfide, } \\
\text { 3-methyl thiophene, } \\
\text { 2,3-butanedione, } \\
\text { 2-nonanoneacetic acid }\end{array}$ & HS-SPME & CAR-PDMS & 180 & 37 & - & - & 5 & 240 & $\begin{array}{l}\text { GC-MS / } \\
\text { GC-O }\end{array}$ & - & - & [209] \\
\hline $\begin{array}{l}\text { Cooked, } \\
\text { fermented } \\
\text { sausage }\end{array}$ & VVC & SPME & CAR-PDMS & 40 & 47 & - & - & 10 & 250 & GC-MS & - & - & [210] \\
\hline $\begin{array}{l}\text { Cooked } \\
\text { beef }\end{array}$ & VVC & HS-SPME & DVB-CAR-PDMS & 25 & 40 & $6 \% \mathrm{NaCl}$ & - & 3 & 250 & GC-MS & - & - & [211] \\
\hline
\end{tabular}


Table 3. Cont.

\begin{tabular}{|c|c|c|c|c|c|c|c|c|c|c|c|c|c|}
\hline \multicolumn{8}{|c|}{ Extraction conditions } & \multicolumn{3}{|c|}{$\begin{array}{l}\text { Desorption } \\
\text { conditions }\end{array}$} & \multirow[b]{2}{*}{ LOD } & \multirow[b]{2}{*}{$\begin{array}{l}\text { Recove } \\
\text { ry }\end{array}$} & \multirow[b]{2}{*}{ Ref. } \\
\hline $\begin{array}{l}\text { Food } \\
\text { Sample }\end{array}$ & Analyte & Technique & Fiber & $\begin{array}{l}\text { Time } \\
{[\text { min] }}\end{array}$ & $\begin{array}{l}\text { Temp. } \\
{\left[{ }^{\circ} \mathrm{C}\right]}\end{array}$ & $\begin{array}{l}\text { Salt } \\
\text { addition }\end{array}$ & Deriv. & $\begin{array}{r}\text { Time } \\
\text { [min] }\end{array}$ & $\begin{array}{l}\text { Temp. } \\
{\left[{ }^{\circ} \mathrm{C}\right]}\end{array}$ & Detection & & & \\
\hline \multicolumn{14}{|c|}{ Juice and alcoholic beverages } \\
\hline Whisky & $\begin{array}{l}\text { fatty acid ethyl esters, } \\
\text { higher alcohols, fatty } \\
\text { acids, carbonyl com- } \\
\text { pounds, } \\
\text { monoterpenols, C13 } \\
\text { norisoprenoids, } \\
\text { volatile phenols }\end{array}$ & HS-SPME & CAR-PDMS & 60 & 40 & $30 \% \mathrm{NaCl}$ & - & 6 & 220 & GC-MS & - & - & [212] \\
\hline $\begin{array}{l}\text { Banana } \\
\text { Terra spirit }\end{array}$ & $\begin{array}{l}\text { 3-methylbutan-1-ol, 3- } \\
\text { methylbutan-1-ol } \\
\text { acetate, 2- } \\
\text { phenylethylacetate, } \\
\text { phenylethyl alcohol }\end{array}$ & HS-SPME & PDMS-CAR-DVB & 25 & 60 & - & - & 2 & 240 & $\begin{array}{l}\text { GC-MS / } \\
\text { GC-O }\end{array}$ & - & - & {$[213]$} \\
\hline $\begin{array}{l}\text { Chinese } \\
\text { Laobaigan } \\
\text { liquor }\end{array}$ & VVC & HS-SPME & $\begin{array}{l}\text { DVB-CAR-PDMS / } \\
\text { CAR-PDMS }\end{array}$ & 40 & 60 & $3 \mathrm{~g} \mathrm{NaCl}$ & - & 5 & 250 & GC-MS & - & - & {$[214]$} \\
\hline Ice wine & aroma compounds & HS-SPME & DVB-CAR-PDMS & 5 & 45 & $1 \mathrm{~g} \mathrm{NaCl}$ & - & 2 & 260 & $\begin{array}{l}\text { GC-TOF- } \\
\text { MS }\end{array}$ & $\begin{array}{l}\text { trans-OL: } \\
0.015 \mathrm{~g} / \mathrm{mL} \text {; } \\
\text { cis-OL: } \\
0.01 \mathrm{~g} / \mathrm{mL}\end{array}$ & - & [215,216] \\
\hline
\end{tabular}


Table 3. Cont.

\begin{tabular}{|c|c|c|c|c|c|c|c|c|c|c|c|c|c|}
\hline \multicolumn{8}{|c|}{ Extraction conditions } & \multicolumn{3}{|c|}{$\begin{array}{l}\text { Desorption } \\
\text { conditions }\end{array}$} & \multirow[b]{2}{*}{ LOD } & \multirow[b]{2}{*}{ Recovery } & \multirow[b]{2}{*}{ Ref. } \\
\hline $\begin{array}{l}\text { Food } \\
\text { Sample }\end{array}$ & Analyte & Technique & Fiber & $\begin{array}{l}\text { Time } \\
\text { [min] }\end{array}$ & $\begin{array}{l}\text { Temp. } \\
{\left[{ }^{\circ} \mathrm{C}\right]}\end{array}$ & $\begin{array}{l}\text { Salt } \\
\text { addition }\end{array}$ & Deriv. & $\begin{array}{l}\text { Time } \\
\text { [min] }\end{array}$ & $\begin{array}{l}\text { Temp. } \\
{\left[{ }^{\circ} \mathrm{C}\right]}\end{array}$ & Detection & & & \\
\hline \multicolumn{14}{|c|}{ Juice and alcoholic beverages } \\
\hline Wine & $\begin{array}{l}\text { 2-methyl-3-furanthiol, } \\
\text { 4-mercapto-4-methyl- } \\
\text { 2-pentanone, } \\
\text { 3-mercaptohexanol, } \\
\text { 2-furanmethane-thiol, } \\
\text { 3-mercaptohexyl } \\
\text { acetate }\end{array}$ & HS-SPME & PDMS-DVB & 10 & 55 & - & $\begin{array}{l}\text { on- } \\
\text { fiber } \\
\text { deri- } \\
\text { vatizati } \\
\text { on }\end{array}$ & 2 & 250 & $\begin{array}{l}\text { GC-NCI- } \\
\text { MS }\end{array}$ & $0.03-0.8 \mathrm{fg}$ & - & {$[85]$} \\
\hline $\begin{array}{l}\text { Spanish } \\
\text { white wines }\end{array}$ & VVC & HS-SPME & CAR-PDMS & 60 & 46 & - & - & 2 & 280 & $\begin{array}{l}\text { GC-MS / } \\
\text { GC-FID }\end{array}$ & $\begin{array}{l}0.1-900 \\
\mathrm{ng} / \mathrm{mL}\end{array}$ & $97-110 \%$ & {$[217]$} \\
\hline $\begin{array}{l}\text { Cherry } \\
\text { wines }\end{array}$ & VVC & HS-SPME & DVB-CAR-PDMS & 45 & 50 & $2 \mathrm{~g} \mathrm{NaCl}$ & - & 5 & 230 & GC-MS & $\begin{array}{l}0.03-7.27 \\
\mu \mathrm{g} / \mathrm{L}\end{array}$ & $\begin{array}{l}60.7- \\
125.6 \%\end{array}$ & {$[218]$} \\
\hline $\begin{array}{l}\text { China } \\
\text { gingko } \\
\text { wine }\end{array}$ & VVC & HS-SPME & CAR-PDMS & 45 & 50 & $\begin{array}{l}\text { saturated } \\
\mathrm{NaCl} \\
\text { solution }\end{array}$ & - & 4 & 250 & GC-MS & - & - & [219] \\
\hline $\begin{array}{l}\text { Black } \\
\text { raspberry } \\
\text { wines }\end{array}$ & VVC & HS-SPME & PDMS-DVB & 30 & 60 & - & - & 5 & 230 & GC-MS & - & - & [220] \\
\hline Beer & $\begin{array}{l}\text { alcohols, esters, } \\
\text { organic acids, } \\
\text { aldehydes, ketones, } \\
\text { terpenes, sulfur com- } \\
\text { pounds, amines, } \\
\text { phenols }\end{array}$ & HS-SPME & $\begin{array}{l}\text { TMSPMA-OH-TSO } \\
\text { prepared by sol-gel } \\
\text { technology }\end{array}$ & 30 & 40 & $2 \mathrm{~g} \mathrm{NaCl}$ & - & 5 & 300 & GC & $\begin{array}{l}0.01-35.2 \\
\mu \mathrm{g} / \mathrm{L}\end{array}$ & $\begin{array}{l}92.8- \\
105.8 \%\end{array}$ & [221] \\
\hline
\end{tabular}


Table 3. Cont.

\begin{tabular}{|c|c|c|c|c|c|c|c|c|c|c|c|c|c|}
\hline \multirow[b]{2}{*}{$\begin{array}{l}\text { Food } \\
\text { Sample } \\
\end{array}$} & \multirow[b]{2}{*}{ Analyte } & \multicolumn{6}{|c|}{ Extraction conditions } & \multicolumn{3}{|c|}{$\begin{array}{l}\text { Desorption } \\
\text { conditions }\end{array}$} & \multirow[b]{2}{*}{ LOD } & \multirow[b]{2}{*}{ Recovery } & \multirow[b]{2}{*}{ Ref. } \\
\hline & & Technique & Fiber & $\begin{array}{l}\text { Time } \\
{[\mathrm{min}]}\end{array}$ & $\begin{array}{l}\text { Temp. } \\
{\left[{ }^{\circ} \mathrm{C}\right]}\end{array}$ & $\begin{array}{l}\text { Salt } \\
\text { addition }\end{array}$ & Deriv. & $\begin{array}{l}\text { Time } \\
{[\mathrm{min}]}\end{array}$ & $\begin{array}{l}\text { Temp. } \\
{\left[{ }^{\circ} \mathrm{C}\right]}\end{array}$ & Detection & & & \\
\hline \multicolumn{14}{|c|}{ Juice and alcoholic beverages } \\
\hline $\begin{array}{l}\text { Yellow } \\
\text { passion } \\
\text { fruit juice }\end{array}$ & VVC & HS-SPME & PDMS-DVB & 20 & 50 & $17 \% \mathrm{NaCl}$ & - & 6 & 250 & GC-MS & - & - & [222] \\
\hline Grape juice & aroma compounds & HS-SBSE & PDMS & 120 & RT & - & - & 5 & -50 & GC-MS & - & $28.4 \%$ & [223] \\
\hline $\begin{array}{l}\text { Orange } \\
\text { beverage } \\
\text { emulsion }\end{array}$ & VVC & HS-SPME & CAR-PDMS & 15 & 45 & $15 \% \mathrm{NaCl}$ & - & 8 & 250 & GC-MS & $\begin{array}{l}0.06-2.27 \\
\mathrm{mg} / \mathrm{L}\end{array}$ & $\begin{array}{l}88.3- \\
121.7 \%\end{array}$ & [224] \\
\hline Coffee & aroma compounds & HS-SPME & PDMS-DVB & 20 & 60 & - & - & 1 & 270 & GC-MS & - & - & [225] \\
\hline Coffee & VVC & HS-SPME & PDMS & 5 & 30 & - & - & - & 220 & SAW & - & - & [226] \\
\hline Coffee & VVC & HS-SPME & DVB-CAR-PDMS & 30 & 60 & - & - & 5 & 230 & $\mathrm{GC}$ & - & - & [227] \\
\hline Coffee & $\begin{array}{l}\text { furans, } \\
\text { methoxyphenols, } \\
\text { pyrazines, and ketones }\end{array}$ & HS-SPME & $\begin{array}{l}\text { poly }\left[\mathrm{VC}_{16} \mathrm{Im}\right]\left[\mathrm{NTf}_{2}\right] \\
\text { with } 50 \% \\
{\left[\mathrm{VBIm}_{2} \mathrm{C}_{12}\right] 2\left[\mathrm{Ntf}_{2}\right]}\end{array}$ & 30 & RT & - & - & 5 & 175 & $\begin{array}{l}\text { GC-MS / } \\
\text { GC-FID }\end{array}$ & - & - & [123] \\
\hline \multicolumn{14}{|c|}{ Fruits and vegetables } \\
\hline $\begin{array}{l}\text { Various } \\
\text { apricot } \\
\text { varieties }\end{array}$ & $\begin{array}{l}\text { ethyl acetate, hexyl } \\
\text { acetate, limonene, } \\
\text { b-cyclocitral, } \\
\text { c-decalactone, } \\
\text { 6-methyl-5-hepten-2- } \\
\text { one, linalool, } \\
\text { b-ionone, menthone } \\
\text { and (E)-hexen-2-al }\end{array}$ & HS-SPME & CAR-PDMS & 20 & 40 & - & - & 4 & 250 & $\begin{array}{l}\text { GC-MS / } \\
\text { GC-O }\end{array}$ & - & - & [228] \\
\hline
\end{tabular}


Table 3. Cont.

\begin{tabular}{|c|c|c|c|c|c|c|c|c|c|c|c|c|c|}
\hline \multirow[b]{2}{*}{$\begin{array}{l}\text { Food } \\
\text { Sample }\end{array}$} & \multirow[b]{2}{*}{ Analyte } & \multicolumn{6}{|c|}{ Extraction conditions } & \multicolumn{3}{|c|}{$\begin{array}{l}\text { Desorption } \\
\text { conditions }\end{array}$} & \multirow[b]{2}{*}{ LOD } & \multirow[b]{2}{*}{ Recovery } & \multirow[b]{2}{*}{ Ref. } \\
\hline & & Technique & Fiber & $\begin{array}{l}\text { Time } \\
\text { [min] }\end{array}$ & $\begin{array}{l}\text { Temp. } \\
{\left[{ }^{\circ} \mathrm{C}\right]}\end{array}$ & $\begin{array}{l}\text { Salt } \\
\text { addition }\end{array}$ & Deriv. & $\begin{array}{l}\text { Time } \\
\text { [min] }\end{array}$ & $\begin{array}{l}\text { Temp. } \\
{\left[{ }^{\circ} \mathrm{C}\right]}\end{array}$ & Detection & & & \\
\hline \multicolumn{14}{|c|}{ Fruits and vegetables } \\
\hline $\begin{array}{l}\text { Apricot } \\
\text { varieties }\end{array}$ & $\begin{array}{l}\text { linalool, a-terpineol, } \\
\text { b-ionone and c- } \\
\text { decalactone }\end{array}$ & HS-SPME & CAR-PDMS & 20 & 40 & $\begin{array}{l}\text { saturated } \\
\mathrm{NaCl} \\
\text { solution }\end{array}$ & - & 4 & 250 & GC-MS & - & - & [229] \\
\hline $\begin{array}{l}\text { Apricot } \\
\text { varieties }\end{array}$ & $\begin{array}{l}\text { aldehydes, alcohols, } \\
\text { acetates, esters, } \\
\text { terpenes and acids }\end{array}$ & HS-SPME & CAR-PDMS & 30 & 40 & - & - & 2 & 250 & GC-MS & - & - & [230] \\
\hline Jackfruit & $\begin{array}{l}\text { ethyl isovalerate, 3- } \\
\text { methylbutyl acetate, } \\
\text { 1-butanol, propyl } \\
\text { isovalerate, isobutyl } \\
\text { isovalerate, 2-methyl- } \\
\text { butanol, butyl } \\
\text { isovalerate }\end{array}$ & HS-SPME & DVB-CAR-PDMS & 30 & 10 & - & - & 5 & 250 & $\begin{array}{l}\text { GC-TOF- } \\
\text { MS }\end{array}$ & - & - & [231] \\
\hline $\begin{array}{l}\text { Cooked } \\
\text { peaches }\end{array}$ & VVC & HS-SPME & DVB-CAR-PDMS & 20 & 40 & - & - & 10 & 270 & GC-MS & - & - & [232] \\
\hline $\begin{array}{l}\text { Monstera } \\
\text { deliciosa } \\
\text { fruit }\end{array}$ & VVC & HS-SPME & PDMS-DVB & 60 & 40 & $15 \% \mathrm{NaCl}$ & - & 6 & 250 & GC-qMS & - & - & [233] \\
\hline $\begin{array}{l}\text { Pineapple } \\
\text { fruit }\end{array}$ & VVC & HS-SPME & PDMS-DVB & 30 & 40 & - & - & 2 & 240 & $\begin{array}{l}\mathrm{GC} \times \mathrm{GC}- \\
\mathrm{qMS}\end{array}$ & - & - & [234] \\
\hline $\begin{array}{l}\text { Sweet } \\
\text { cherry } \\
\text { cultivars }\end{array}$ & VVC & HS-SPME & CAR-PDMS & 20 & 45 & $0.2 \mathrm{~g} \mathrm{NaCl}$ & - & 10 & 260 & GC-MS & - & - & [235] \\
\hline
\end{tabular}


Table 3. Cont.

\begin{tabular}{|c|c|c|c|c|c|c|c|c|c|c|c|c|c|}
\hline & & & Extraction & nditior & & & & $\begin{array}{l}\text { Deso } \\
\text { cond }\end{array}$ & $\begin{array}{l}\text { rption } \\
\text { ditions }\end{array}$ & & & & \\
\hline $\begin{array}{l}\text { Food } \\
\text { Sample }\end{array}$ & Analyte & Technique & Fiber & $\begin{array}{l}\text { Time } \\
\text { [min] }\end{array}$ & $\begin{array}{l}\text { Temp. } \\
{\left[{ }^{\circ} \mathrm{C}\right]}\end{array}$ & $\begin{array}{l}\text { Salt } \\
\text { addition }\end{array}$ & Deriv. & $\begin{array}{l}\text { Time } \\
{[\mathrm{min}]}\end{array}$ & $\begin{array}{l}\text { Temp. } \\
{\left[{ }^{\circ} \mathrm{C}\right]}\end{array}$ & Detection & LOD & Recovery & Ref. \\
\hline \multicolumn{14}{|c|}{ Fruits and vegetables } \\
\hline $\begin{array}{l}\text { Air-dried } \\
\text { raisins }\end{array}$ & $\begin{array}{l}\text { free and glycosidically } \\
\text { bound volatile } \\
\text { compounds }\end{array}$ & HS-SPME & CAR-PDMS-DVB & 40 & 60 & $1.3 \mathrm{~g} \mathrm{NaCl}$ & - & 8 & - & GC-MS & - & - & [236] \\
\hline $\begin{array}{l}\text { Table } \\
\text { grapes }\end{array}$ & $\begin{array}{l}\text { alcohols, carbonyls, } \\
\text { C6 compounds, } \\
\text { terpenoids, esters }\end{array}$ & HS-SPME & PDMS-DVB & 30 & 40 & $\begin{array}{l}2 \mathrm{~g} \mathrm{CaCl}_{2}, \\
20 \mathrm{~g} \mathrm{NaCl}\end{array}$ & - & 3 & 220 & GC-MS & - & - & [237] \\
\hline Tomato & VVC & HS-SPME & DVB-CAR-PDMS & 15 & 50 & $\begin{array}{l}\text { saturated } \\
\mathrm{CaCl}_{2} \\
\text { solution } \\
\end{array}$ & - & 10 & 250 & GC-MS & - & - & [238] \\
\hline \multicolumn{14}{|c|}{ Miscellaneous } \\
\hline $\begin{array}{l}\text { Thistle } \\
\text { honey }\end{array}$ & VVC & HS-SPME & DVB-CAR-PDMS & 40 & 60 & $30 \% \mathrm{NaCl}$ & - & 2 & 250 & GC-MS & - & - & [239] \\
\hline $\begin{array}{l}\text { Croatian } \\
\text { lime tree, } \\
\text { fir honey- } \\
\text { dew, sage } \\
\text { honey }\end{array}$ & VVC & HS-SPME & DVB-CAR-PDMS & 20 & 40 & $\begin{array}{l}0.5 \mathrm{~g} \text { an- } \\
\text { hydrous } \\
\mathrm{Na}_{2} \mathrm{SO}_{4}\end{array}$ & - & 3 & 250 & GC-MS & - & - & [240] \\
\hline Honey & VVC & HS-SPME & PDMS-DVB & 40 & 50 & - & - & 2 & 250 & $\begin{array}{l}\text { GC- } \\
\text { QTOF- } \\
\text { MS }\end{array}$ & - & - & [241] \\
\hline $\begin{array}{l}\text { Extra virgin } \\
\text { olive oils }\end{array}$ & VVC & HS-SPME & DVB-CAR-PDMS & 10 & 40 & - & - & 5 & 260 & GC-FID & - & - & [242] \\
\hline $\begin{array}{l}\text { Extra virgin } \\
\text { olive oils }\end{array}$ & VVC & HS-SPME & DVB-CAR-PDMS & 360 & 30 & - & - & 4 & 270 & GC-MS & - & - & [243] \\
\hline
\end{tabular}


Table 3. Cont

\begin{tabular}{|c|c|c|c|c|c|c|c|c|c|c|c|c|c|}
\hline \multirow[b]{2}{*}{$\begin{array}{l}\text { Food } \\
\text { Sample }\end{array}$} & \multirow[b]{2}{*}{ Analyte } & \multicolumn{6}{|c|}{ Extraction conditions } & \multicolumn{3}{|c|}{$\begin{array}{l}\text { Desorption } \\
\text { conditions }\end{array}$} & \multirow[b]{2}{*}{ LOD } & \multirow[b]{2}{*}{ Recovery } & \multirow[b]{2}{*}{ Ref. } \\
\hline & & Technique & Fiber & $\begin{array}{l}\text { Time } \\
\text { [min] }\end{array}$ & $\begin{array}{l}\text { Temp. } \\
{\left[{ }^{\circ} \mathrm{C}\right]}\end{array}$ & $\begin{array}{l}\text { Salt } \\
\text { addition }\end{array}$ & Deriv. & $\begin{array}{l}\text { Time } \\
\text { [min] }\end{array}$ & $\begin{array}{l}\text { Temp. } \\
{\left[{ }^{\circ} \mathrm{C}\right]}\end{array}$ & Detection & & & \\
\hline \multicolumn{14}{|c|}{ Miscellaneous } \\
\hline $\begin{array}{l}\text { Virgin olive } \\
\text { oil }\end{array}$ & VVC & HS-SPME & DVB-CAR-PDMS & 40 & 40 & - & - & 5 & 300 & GC-MS & $\begin{array}{l}0.1-2.54 \\
\mathrm{mg} / \mathrm{kg}\end{array}$ & - & [244] \\
\hline $\begin{array}{l}\text { Black and } \\
\text { white rice } \\
\text { bran }\end{array}$ & $\begin{array}{l}\text { terpenoid flavor } \\
\text { odorants }\end{array}$ & HS-SPME & PDMS & 30 & 100 & - & - & 0.2 & 250 & $\begin{array}{l}\text { GCxGC- } \\
\text { MS }\end{array}$ & - & - & [245] \\
\hline $\begin{array}{l}\text { Italian rice } \\
\text { cultivars }\end{array}$ & VVC & HS-SPME & DVB-CAR-PDMS & 60 & 60 & - & - & 5 & 250 & GC-MS & - & - & [246] \\
\hline Palm sugar & $\begin{array}{l}\text { N-heterocyclic and } \\
\text { O-heterocyclic } \\
\text { compounds }\end{array}$ & HS-SPME & DVB-CAR-PDMS & 10 & 50 & - & - & 5 & 240 & GC-MS & - & - & [247] \\
\hline $\begin{array}{l}\text { Almond } \\
\text { cultivars }\end{array}$ & VVC & HS-SPME & DVB-CAR-PDMS & 60 & 60 & - & - & 10 & 270 & GC-MS & - & - & [248] \\
\hline Saffron & VVC & HS-SPME & PDMS & 20 & 36 & - & - & 0.4 & 250 & GC-MS & - & - & [249] \\
\hline Garlic & VVC & HS-SPME & CAR-PDMS & 30 & 30 & - & - & 3 & 220 & GC-MS & - & - & [250] \\
\hline $\begin{array}{l}\text { Atlantic } \\
\text { shellfish } \\
\text { species }\end{array}$ & VVC & HS-SPME & CAR-PDMS & 30 & 80 & $\begin{array}{l}10 \mathrm{~mL} \\
\text { saturated } \\
\mathrm{NaCl} \\
\text { solution }\end{array}$ & - & 10 & 260 & GC-MS & $\begin{array}{l}0.12-1.19 \\
\mathrm{ppb}\end{array}$ & $\begin{array}{l}59.3- \\
119.6 \%\end{array}$ & [251] \\
\hline
\end{tabular}


Table 3. Cont.

\begin{tabular}{|c|c|c|c|c|c|c|c|c|c|c|c|c|c|}
\hline & & & Extraction co & nditio & & & & $\begin{array}{l}\text { Deso } \\
\text { cond }\end{array}$ & $\begin{array}{l}\text { rption } \\
\text { litions }\end{array}$ & & & & \\
\hline $\begin{array}{l}\text { Food } \\
\text { Sample } \\
\end{array}$ & Analyte & Technique & Fiber & $\begin{array}{l}\text { Time } \\
{[\text { min] }}\end{array}$ & $\begin{array}{l}\text { Temp. } \\
{\left[{ }^{\circ} \mathbf{C}\right]}\end{array}$ & $\begin{array}{l}\text { Salt } \\
\text { addition }\end{array}$ & Deriv. & $\begin{array}{l}\text { Time } \\
{[\mathrm{min}]}\end{array}$ & $\begin{array}{l}\text { Temp. } \\
{\left[{ }^{\circ} \mathrm{C}\right]}\end{array}$ & Detection & LOD & Recovery & Ref. \\
\hline \multicolumn{14}{|c|}{ Food products } \\
\hline $\begin{array}{l}\text { Beer and } \\
\text { beverage }\end{array}$ & sulfur compounds & $\begin{array}{l}\text { HS- } \\
\text { SDME / } \\
\text { DI-SPME } \\
\text { / HS- } \\
\text { SDME }\end{array}$ & PDMS & 5 & 25 & $\begin{array}{l}2.0 \mathrm{~g} \mathrm{NaCl} \\
/ 20 \% \\
\mathrm{NaCl} \\
\text { solution }\end{array}$ & - & 5 & 250 & GC-FPD & $\begin{array}{l}0.5 \mathrm{ng} / \mathrm{mL} \\
\text { for DPrDS } \\
-208.1 \\
\mathrm{ng} / \mathrm{mL}\end{array}$ & $\begin{array}{l}\text { beer: } \\
85.5- \\
106.9 \% \text {; } \\
\text { bevera- } \\
\text { ges: } \\
95.2- \\
110.8 \%\end{array}$ & [252] \\
\hline Beer & $\begin{array}{l}\text { esters and vicinal } \\
\text { diketones }\end{array}$ & HS-SPME & DVB-CAR-PDMS & 30 & 60 & $3.0 \mathrm{~g} \mathrm{NaCl}$ & - & - & 260 & GC-MS & - & - & [253] \\
\hline $\begin{array}{l}\text { Orange } \\
\text { juice }\end{array}$ & $\begin{array}{l}\text { guaiacol and } \\
\text { halogenated phenol }\end{array}$ & HS-SPME & DVB-CAR-PDMS & 30 & 40 & - & - & 5 & 220 & $\begin{array}{l}\text { GC-MS / } \\
\text { GC-O }\end{array}$ & - & - & [256] \\
\hline $\begin{array}{l}\text { Water and } \\
\text { apple juice }\end{array}$ & geosmin & HS-SPME & $\begin{array}{l}\text { PDMS synthesized as } \\
\text { coated fiber by sol- } \\
\text { gel technology }\end{array}$ & 25 & 40 & $37 \% \mathrm{NaCl}$ & - & 4 & 250 & GC-MS & $\begin{array}{l}1-1.000 \\
\mathrm{ng} / \mathrm{L}\end{array}$ & $95-102 \%$ & [257] \\
\hline $\begin{array}{l}\text { Coffee } \\
\text { beverage }\end{array}$ & $\begin{array}{l}\text { volatile compounds } \\
\text { produced by fungi }\end{array}$ & HS-SPME & DVB-CAR & 30 & 65 & - & - & 0.7 & 270 & GC-MS & - & - & [258] \\
\hline
\end{tabular}


Table 3. Cont

\begin{tabular}{|c|c|c|c|c|c|c|c|c|c|c|c|c|c|}
\hline \multirow[b]{2}{*}{$\begin{array}{l}\text { Food } \\
\text { Sample }\end{array}$} & \multirow[b]{2}{*}{ Analyte } & \multicolumn{6}{|c|}{ Extraction conditions } & \multicolumn{3}{|c|}{$\begin{array}{l}\text { Desorption } \\
\text { conditions }\end{array}$} & \multirow[b]{2}{*}{ LOD } & \multirow[b]{2}{*}{ Recovery } & \multirow[b]{2}{*}{ Ref. } \\
\hline & & Technique & Fiber & $\begin{array}{l}\text { Time } \\
\text { [min] }\end{array}$ & $\begin{array}{l}\text { Temp. } \\
{\left[{ }^{\circ} \mathrm{C}\right]}\end{array}$ & $\begin{array}{l}\text { Salt } \\
\text { addition }\end{array}$ & Deriv. & $\begin{array}{l}\text { Time } \\
\text { [min] }\end{array}$ & $\begin{array}{l}\text { Temp. } \\
{\left[{ }^{\circ} \mathrm{C}\right]}\end{array}$ & Detection & & & \\
\hline \multicolumn{14}{|c|}{ Off-Flavor } \\
\hline \multicolumn{14}{|c|}{ Food products } \\
\hline $\begin{array}{l}\text { Rapeseed } \\
\text { oil }\end{array}$ & $\begin{array}{l}\text { hexanal, } \\
\text { 2,4-heptadienal, } \\
\text { 2-heptenal and } \\
\text { 1-pentene-3-ol }\end{array}$ & HS-SPME & CAR-DVB-PDMS & 35 & 50 & - & - & - & 150 & MS & - & - & [259] \\
\hline $\begin{array}{l}\text { Convention } \\
\text { al and high- } \\
\text { oleic } \\
\text { sunflower } \\
\text { oil }\end{array}$ & $\begin{array}{l}\text { hexanal, }(\mathrm{E})-2- \\
\text { heptenal, }(\mathrm{E})-2- \\
\text { decenal, }(\mathrm{E}, \mathrm{E})-2,4- \\
\text { nonadienal }\end{array}$ & HS-SPME & CAR-DVB-PDMS & $\begin{array}{l}60 / 90 \\
/ 120\end{array}$ & $\begin{array}{l}40 / 60 \\
/ 80\end{array}$ & - & - & 5 & 270 & MS & $\begin{array}{l}0.4-4.3 \\
\mathrm{mg} / \mathrm{L}\end{array}$ & - & [260] \\
\hline $\begin{array}{l}\text { Convention } \\
\text { al and high- } \\
\text { oleic } \\
\text { rapeseed oil }\end{array}$ & $\begin{array}{l}\text { octanal, 3-octanone, } \\
\text { propanal, (E,E)-2,4- } \\
\text { hexa-dienal, (E)-2- } \\
\text { heptenal }\end{array}$ & HS-SPME & CAR-DVB-PDMS & 90 & 40 & - & - & 5 & 270 & MS & $\begin{array}{l}3.7-816.5 \\
\mu \mathrm{g} / \mathrm{L}\end{array}$ & - & [261] \\
\hline $\begin{array}{l}\text { Various } \\
\text { frying oils }\end{array}$ & $\begin{array}{l}(\mathrm{E}, \mathrm{E})-2,4-\text { decadienal, } \\
\text { heptanal, }(\mathrm{E}, \mathrm{E})-2,4- \\
\text { heptadienal, }(\mathrm{E})-2- \\
\text { decenal }\end{array}$ & HS-SPME & CAR-DVB-PDMS & 90 & 40 & - & - & 5 & 270 & MS & $\begin{array}{l}0.03-47.2 \\
\mu \mathrm{g} / \mathrm{L}\end{array}$ & - & [262] \\
\hline $\begin{array}{l}\text { Almond } \\
\text { oils }\end{array}$ & $\begin{array}{l}\text { hexanal, }(\mathrm{E})-2- \\
\text { heptenal, }(\mathrm{E})-2- \\
\text { octenal, nonanal, }(\mathrm{E})- \\
\text { 2-nonenal, }(\mathrm{E}, \mathrm{E})-2,4- \\
\text { nonadienal, }(\mathrm{E}, \mathrm{E})-2,4- \\
\text { decadienal }\end{array}$ & HS-SPME & DVB-CAR-PDMS & 60 & 60 & - & - & 10 & 270 & GC-MS & - & - & [263] \\
\hline
\end{tabular}


Table 3. Cont.

\begin{tabular}{|c|c|c|c|c|c|c|c|c|c|c|c|c|c|}
\hline \multirow[b]{2}{*}{$\begin{array}{l}\text { Food } \\
\text { Sample } \\
\end{array}$} & \multirow[b]{2}{*}{ Analyte } & \multicolumn{6}{|c|}{ Extraction conditions } & \multicolumn{3}{|c|}{$\begin{array}{c}\text { Desorption } \\
\text { conditions } \\
\end{array}$} & \multirow[b]{2}{*}{ LOD } & \multirow[b]{2}{*}{ Recovery } & \multirow[b]{2}{*}{ Ref. } \\
\hline & & Technique & Fiber & $\begin{array}{l}\text { Time } \\
\text { [min] }\end{array}$ & $\begin{array}{l}\text { Temp. } \\
{\left[{ }^{\circ} \mathrm{C}\right]}\end{array}$ & $\begin{array}{l}\text { Salt } \\
\text { addition }\end{array}$ & Deriv. & $\begin{array}{l}\text { Time } \\
\text { [min] }\end{array}$ & $\begin{array}{l}\text { Temp. } \\
{\left[{ }^{\circ} \mathrm{C}\right]}\end{array}$ & Detection & & & \\
\hline \multicolumn{14}{|c|}{ Off-Flavor } \\
\hline \multicolumn{14}{|c|}{ Food products } \\
\hline Butter & hexanal & HS-SPME & CAR-PDMS & 180 & 4 & - & - & 5 & 250 & GC-MS & - & $97.37 \%$ & {$[264]$} \\
\hline Soymilk & $\begin{array}{l}\text { aldehydes, alcohols, } \\
\text { ketones, aromatic } \\
\text { compounds, esters, } \\
\text { furans }\end{array}$ & HS-SPME & CAR-PDMS & 20 & 40 & - & - & 3 & 300 & GC-MS & - & - & {$[265]$} \\
\hline $\begin{array}{l}\text { Fresh } \\
\text { chilled } \\
\text { pasteurised } \\
\text { milk }\end{array}$ & $\begin{array}{l}\text { microbially induced } \\
\text { changes in volatile } \\
\text { constituents }\end{array}$ & HS-SPME & CAR-PDMS & 30 & 40 & - & - & 2 & 240 & $\begin{array}{l}\text { GC-MS / } \\
\text { PTR-MS }\end{array}$ & - & - & {$[266]$} \\
\hline $\begin{array}{l}\text { Full fat } \\
\text { bovine milk }\end{array}$ & $\begin{array}{l}\text { volatile compounds } \\
\text { (pentanal, pentanol, } \\
\text { hexanal) produced by } \\
\text { photooxidation }\end{array}$ & HS-SPME & CAR-PDMS & 30 & 50 & - & - & 0.02 & 250 & GC-MS & - & - & {$[267]$} \\
\hline $\begin{array}{l}\text { Chicken } \\
\text { breast }\end{array}$ & $\begin{array}{l}\text { sulfides methanethiol, } \\
\text { dimethyl disulfide, di- } \\
\text { methyl trisulfide, } \\
\text { ethanol, 1- and 2- } \\
\text { butanol, 1-butanol } \\
\text { isomers, free fatty } \\
\text { acids }\end{array}$ & HS-SPME & PDMS & 15 & 50 & - & - & 3 & 200 & $\begin{array}{l}\text { GC-MS- } \\
\text { FASST }\end{array}$ & - & - & {$[268]$} \\
\hline
\end{tabular}


Table 3. Cont.

\begin{tabular}{|c|c|c|c|c|c|c|c|c|c|c|c|c|c|}
\hline & & & Extraction & ndition & & & & $\begin{array}{l}\text { Deso } \\
\text { cond }\end{array}$ & $\begin{array}{l}\text { rption } \\
\text { litions }\end{array}$ & & & & \\
\hline $\begin{array}{l}\text { Food } \\
\text { Sample } \\
\end{array}$ & Analyte & Technique & Fiber & $\begin{array}{l}\text { Time } \\
{[\mathrm{min}]}\end{array}$ & $\begin{array}{l}\text { Temp. } \\
{\left[{ }^{\circ} \mathrm{C}\right]}\end{array}$ & $\begin{array}{l}\text { Salt } \\
\text { addition }\end{array}$ & Deriv. & $\begin{array}{l}\text { Time } \\
{[\mathrm{min}]}\end{array}$ & $\begin{array}{l}\text { Temp. } \\
{\left[{ }^{\circ} \mathrm{C}\right]}\end{array}$ & Detection & LOD & Recovery & Ref. \\
\hline \multicolumn{14}{|c|}{ Off-Flavor } \\
\hline \multicolumn{14}{|c|}{ Food products } \\
\hline Whiting & $\begin{array}{l}\text { trimethylamine, 3- } \\
\text { methyl-butanal, 2- } \\
\text { methyl-butanal, 3- } \\
\text { hydroxy-2-butanone, } \\
\text { 3-methyl-1-butanol, 2- } \\
\text { methyl-1-butanol }\end{array}$ & HS-SPME & CAR-PDMS & 40 & 50 & $\begin{array}{l}\text { saturated } \\
\mathrm{NaCl} \\
\text { solution }\end{array}$ & - & 0.16 & 250 & GC-MS & - & - & [269] \\
\hline $\begin{array}{l}\text { Rainbow } \\
\text { trout }\end{array}$ & geosmin & HS-SPME & DVB-PDMS & 20 & 65 & $3.0 \mathrm{~g} \mathrm{NaCl}$ & - & 3 & 270 & GC-MS & - & - & {$[270]$} \\
\hline $\begin{array}{l}\text { Potato } \\
\text { crisps }\end{array}$ & VVC & HS-SPME & CAR-PDMS & 20 & 50 & - & - & 3 & 300 & $\begin{array}{l}\text { MS } \\
\text { e-nose / } \\
\text { GS e-nose }\end{array}$ & - & - & [271] \\
\hline \multicolumn{14}{|c|}{ Food packaging migrants } \\
\hline Cork & chloroanisoles & $\begin{array}{l}\text { CF-HS- } \\
\text { SPME }\end{array}$ & PDMS & 10 & $\begin{array}{l}130 / \\
10\end{array}$ & - & - & 3 & 260 & $\begin{array}{l}\text { GC-TOF- } \\
\text { MS }\end{array}$ & - & $>90 \%$ & {$[56]$} \\
\hline Wine & $\begin{array}{l}\text { chlorophenols and } \\
\text { chloroanisoles }\end{array}$ & $\begin{array}{l}\text { MHS- } \\
\text { SPME }\end{array}$ & DVB-CAR-PDMS & 60 & 70 & - & $\begin{array}{l}\mathrm{KHCO}_{3} \\
\text { and } \\
\text { acetic } \\
\text { acid } \\
\text { anhy- } \\
\text { dride }\end{array}$ & 5 & 280 & $\begin{array}{l}\text { GC-MS- } \\
\text { MS }\end{array}$ & $\begin{array}{l}0.004- \\
0.077 \mathrm{ng}\end{array}$ & - & {$[80]$} \\
\hline Wine & $\begin{array}{l}\text { 4-ethylphenol, 4- } \\
\text { ethylguaiacol }\end{array}$ & SPME & PDMS-CAR & 30 & 60 & - & - & 15 & 220 & GC-MS & - & - & [272] \\
\hline
\end{tabular}


Table 3. Cont

\begin{tabular}{|c|c|c|c|c|c|c|c|c|c|c|c|c|c|}
\hline \multirow[b]{2}{*}{$\begin{array}{l}\text { Food } \\
\text { Sample }\end{array}$} & \multirow[b]{2}{*}{ Analyte } & \multicolumn{6}{|c|}{ Extraction conditions } & \multicolumn{3}{|c|}{$\begin{array}{l}\text { Desorption } \\
\text { conditions }\end{array}$} & \multirow[b]{2}{*}{ LOD } & \multirow[b]{2}{*}{ Recovery } & \multirow[b]{2}{*}{ Ref. } \\
\hline & & Technique & Fiber & $\begin{array}{l}\text { Time } \\
\text { [min] }\end{array}$ & $\begin{array}{l}\text { Temp. } \\
{\left[{ }^{\circ} \mathrm{C}\right]}\end{array}$ & $\begin{array}{l}\text { Salt } \\
\text { addition }\end{array}$ & Deriv. & $\begin{array}{l}\text { Time } \\
\text { [min] }\end{array}$ & $\begin{array}{l}\text { Temp. } \\
{\left[{ }^{\circ} \mathrm{C}\right]}\end{array}$ & Detection & & & \\
\hline \multicolumn{14}{|c|}{ Food packaging migrants } \\
\hline Wine & $\begin{array}{l}\text { 2,4,6-trichloroanisole, } \\
\text { 2,3,4,6-tetrachloroani- } \\
\text { sole, } \\
\text { pentachloroanisole, } \\
\text { 2,4,6-tribromoanisole, } \\
\text { 4-ethylphenol (4-EP), } \\
\text { 4-ethylguaiacol, 4- } \\
\text { vinyl-phenol, 4- } \\
\text { vinylguaiacol }\end{array}$ & $\begin{array}{l}\text { MHS- } \\
\text { SPME }\end{array}$ & DVB-CAR-PDMS & 60 & 70 & - & - & 5 & 270 & $\begin{array}{l}\text { GC-MS- } \\
\text { MS }\end{array}$ & $\begin{array}{l}\text { 4-EP: } \\
1800 \mathrm{~g} / \mathrm{L} ; \\
\text { others: } \\
1000 \mathrm{~g} / \mathrm{L}\end{array}$ & $\begin{array}{l}93.85- \\
101.27 \%\end{array}$ & {$[273]$} \\
\hline Wine & haloanisoles & $\begin{array}{l}\text { MHS- } \\
\text { SPME }\end{array}$ & DVB-CAR-PDMS & 35 & 60 & $\begin{array}{l}99.8 \% \\
\mathrm{NaCl}\end{array}$ & - & 4 & 250 & $\begin{array}{l}\text { GC-ion- } \\
\text { trap MS }\end{array}$ & $\begin{array}{l}120.70- \\
150 \mathrm{pg}\end{array}$ & $88.8 \%$ & {$[274]$} \\
\hline $\begin{array}{l}\text { Water and } \\
\text { honey }\end{array}$ & chlorophenols & $\begin{array}{l}\text { DMSPE- } \\
\text { HS-SPME }\end{array}$ & $\begin{array}{l}\text { PVC/MWCNTs } \\
\text { nanocomposite }\end{array}$ & 15 & 60 & $\begin{array}{l}5 \mathrm{~mol} / \mathrm{L} \\
\mathrm{NaCl} \\
\text { solution }\end{array}$ & - & 4 & 215 & GC-ECD & $\begin{array}{l}0.08-0.6 \\
\mathrm{ng} / \mathrm{mL}\end{array}$ & $91-109 \%$ & {$[120]$} \\
\hline \multicolumn{14}{|c|}{ Volatlile toxic compounds } \\
\hline \multicolumn{14}{|c|}{ Contaminants } \\
\hline Milk & diethylstilbestrol & DI-SPME & $\begin{array}{l}\text { CNT reinforced } \\
\text { hollow fiber }\end{array}$ & 30 & 60 & - & - & 10 & - & HPLC & $5.1 \mathrm{mg} / \mathrm{L}$ & $\begin{array}{l}57.50 \%- \\
120.42 \%\end{array}$ & {$[125]$} \\
\hline Milk & $\begin{array}{l}\text { enzyme-generated } \\
\text { volatile organic com- } \\
\text { pounds associated } \\
\text { with Listeria } \\
\text { monocytogenes }\end{array}$ & HS-SPME & PA & 10 & 37 & - & - & 2 & 230 & GC-MS & - & - & {$[275]$} \\
\hline
\end{tabular}


Table 3. Cont.

\begin{tabular}{|c|c|c|c|c|c|c|c|c|c|c|c|c|c|}
\hline \multicolumn{8}{|c|}{ Extraction conditions } & \multicolumn{3}{|c|}{$\begin{array}{c}\text { Desorption } \\
\text { conditions }\end{array}$} & \multirow[b]{2}{*}{ LOD } & \multirow[b]{2}{*}{ Recovery } & \multirow[b]{2}{*}{ Ref. } \\
\hline $\begin{array}{l}\text { Food } \\
\text { Sample } \\
\end{array}$ & Analyte & Technique & Fiber & $\begin{array}{l}\text { Time } \\
\text { [min] }\end{array}$ & $\begin{array}{l}\text { Temp. } \\
{\left[{ }^{\circ} \mathrm{C}\right]}\end{array}$ & $\begin{array}{l}\text { Salt } \\
\text { addition }\end{array}$ & Deriv. & $\begin{array}{l}\text { Time } \\
\text { [min] }\end{array}$ & $\begin{array}{l}\text { Temp. } \\
{\left[{ }^{\circ} \mathrm{C}\right]}\end{array}$ & Detection & & & \\
\hline \multicolumn{14}{|c|}{ Volatlile toxic compounds } \\
\hline \multicolumn{14}{|c|}{ Contaminants } \\
\hline Milk & PAHs & DI-SPME & $\begin{array}{l}\text { diethoxydiphenyl- } \\
\text { silane prepared by } \\
\text { sol-gel technology }\end{array}$ & 60 & 60 & - & - & 2 & 330 & GC-MS & $\begin{array}{l}0.01-0.08 \\
\mu \mathrm{g} / \mathrm{L}\end{array}$ & - & [276] \\
\hline $\begin{array}{l}\text { Milk and } \\
\text { honey }\end{array}$ & benzimidazole & DI-SPME & MEMF & 70 & - & - & - & 20 & - & $\begin{array}{l}\text { HPLC- } \\
\text { DAD }\end{array}$ & $\begin{array}{l}0.11-0.30 \\
\mu \mathrm{g} / \mathrm{L}\end{array}$ & $\begin{array}{l}72.3-121 \% \\
83.1-119 \%\end{array}$ & [277] \\
\hline $\begin{array}{l}\text { Chicken } \\
\text { muscle and } \\
\text { milk }\end{array}$ & $\begin{array}{l}\text { tetracyclines } \\
\text { (antibiotic) }\end{array}$ & SPME & $\begin{array}{l}\text { molecularly } \\
\text { imprinted polymer }\end{array}$ & 30 & - & - & - & 10 & - & HPLC & $\begin{array}{l}1.0-2.3 \\
\mu \mathrm{g} / \mathrm{L}\end{array}$ & - & [141] \\
\hline $\begin{array}{l}\text { Baby } \\
\text { formula }\end{array}$ & $\begin{array}{l}\text { furfural and } \\
\text { hydroxymethylfurfura } \\
1\end{array}$ & HS-SPME & $\begin{array}{l}\text { dodecylbenzene- } \\
\text { sulfonate-doped } \\
\text { polypyrrole }\end{array}$ & 30 & 50 & $\begin{array}{l}2 \mathrm{~mol} / \mathrm{L} \\
\mathrm{NaCl}\end{array}$ & - & - & 200 & IMS & $\begin{array}{l}6 \mathrm{ng} / \mathrm{g} \\
5 \mathrm{ng} / \mathrm{g}\end{array}$ & $\begin{array}{l}95 \% / \\
92 \%\end{array}$ & [278] \\
\hline $\begin{array}{l}\text { Baby food } \\
\text { and fruit } \\
\text { juice }\end{array}$ & furan & HS-SPME & $\begin{array}{l}\text { PEG and PEG/CNTs } \\
\text { fibers prepared by } \\
\text { sol-gel technology }\end{array}$ & & $25 / 30$ & $3 \mathrm{~g} \mathrm{NaCl}$ & - & 0.25 & 230 & GC-FID & $\begin{array}{l}0.001 \\
\mathrm{ng} / \mathrm{mL} ; \\
0.00025 \\
\mathrm{ng} / \mathrm{mL}\end{array}$ & $92-98.5 \%$ & {$[126]$} \\
\hline Fruit juices & $\begin{array}{l}\text { carbamate and } \\
\text { phenylurea pesticide } \\
\text { residues }\end{array}$ & DI-SPME & $\begin{array}{l}\text { PDMS-DVB and } \\
\text { CW-TPR }\end{array}$ & 90 & 20 & $\begin{array}{l}0.3 \mathrm{~g} 30 \% \\
\mathrm{NaCl}\end{array}$ & - & 15 & 250 & $\begin{array}{l}\text { LC/QIT- } \\
\mathrm{MS}\end{array}$ & $\begin{array}{l}0.001- \\
0.01 \\
\mathrm{mg} / \mathrm{kg}\end{array}$ & $0-82 \%$ & [279] \\
\hline
\end{tabular}


Table 3. Cont.

\begin{tabular}{|c|c|c|c|c|c|c|c|c|c|c|c|c|c|}
\hline \multicolumn{8}{|c|}{ Extraction conditions } & \multicolumn{3}{|c|}{$\begin{array}{c}\text { Desorption } \\
\text { conditions }\end{array}$} & \multirow[b]{2}{*}{ LOD } & \multirow[b]{2}{*}{ Recovery } & \multirow[b]{2}{*}{ Ref. } \\
\hline $\begin{array}{l}\text { Food } \\
\text { Sample } \\
\end{array}$ & Analyte & Technique & Fiber & $\begin{array}{l}\text { Time } \\
\text { [min] }\end{array}$ & $\begin{array}{l}\text { Temp. } \\
{\left[{ }^{\circ} \mathrm{C}\right]}\end{array}$ & $\begin{array}{l}\text { Salt } \\
\text { addition } \\
\end{array}$ & Deriv. & $\begin{array}{l}\text { Time } \\
\text { [min] }\end{array}$ & $\begin{array}{l}\text { Temp. } \\
{\left[{ }^{\circ} \mathrm{C}\right]}\end{array}$ & Detection & & & \\
\hline \multicolumn{14}{|c|}{ Volatlile toxic compounds } \\
\hline \multicolumn{14}{|c|}{ Contaminants } \\
\hline Soft drinks & 4-methylimidazole & HS-SPME & PDMS-DVB & 50 & 110 & $\begin{array}{l}\text { saturated } \\
\mathrm{NaCl} \\
\text { solution }\end{array}$ & - & - & 270 & $\begin{array}{l}\text { GC-MS / } \\
\text { LC- } \\
\text { MS/MS }\end{array}$ & $1.9 \mu \mathrm{g} / \mathrm{L}$ & - & [280] \\
\hline $\begin{array}{l}\text { Brazilian } \\
\text { sugarcane } \\
\text { juice }\end{array}$ & $\begin{array}{l}\text { pesticide } \\
\text { and benzo[a]pyrene }\end{array}$ & $\begin{array}{l}\text { SBSE and } \\
\text { MASE }\end{array}$ & - & $\begin{array}{l}180 / \\
30\end{array}$ & $\begin{array}{l}280 / \\
45\end{array}$ & $\begin{array}{l}\text { saturated } \\
\mathrm{NaCl} \\
\text { solution }\end{array}$ & - & 11 & 250 & $\begin{array}{l}\text { TD-GC- } \\
\text { MS / LVI- } \\
\text { GC-MS }\end{array}$ & $\begin{array}{l}0.002-0.4 \\
\mu \mathrm{g} / \mathrm{L} \\
0.004- \\
0.56 \mu \mathrm{g} / \mathrm{L}\end{array}$ & $\begin{array}{l}0.2-55.3 \% \\
13.6- \\
103.1 \%\end{array}$ & [281] \\
\hline $\begin{array}{l}\text { Carbonated } \\
\text { drink, juice } \\
\text { drink, } \\
\text { sauce, jam, } \\
\text { succade }\end{array}$ & $\begin{array}{l}\text { benzoic and sorbic } \\
\text { acids }\end{array}$ & $\begin{array}{l}\text { in-tube } \\
\text { SPME }\end{array}$ & $\begin{array}{l}\text { diethylamine- } \\
\text { modified poly(GMA- } \\
\text { co-EDMA) } \\
\text { monolithic capillary }\end{array}$ & 5 & - & - & - & 7 & - & $\begin{array}{l}\text { HPLC- } \\
\text { UV }\end{array}$ & $\begin{array}{l}1.2 ; \\
0.9 \mathrm{ng} / \mathrm{mL}\end{array}$ & $\begin{array}{l}84.4- \\
106 \%\end{array}$ & [282] \\
\hline $\begin{array}{l}\text { Water and } \\
\text { juice }\end{array}$ & $\begin{array}{l}\text { benzoylurea } \\
\text { insecticides }\end{array}$ & $\begin{array}{l}\text { MMF- } \\
\text { SPME }\end{array}$ & MMF/MAED & 70 & - & - & - & - & - & $\begin{array}{l}\text { HPLC- } \\
\text { DAD }\end{array}$ & $\begin{array}{l}\text { water: } \\
0.026- \\
0.075 \\
\mathrm{mg} / \mathrm{L} ; \\
\text { juice: } \\
0.053-0.29 \\
\mathrm{mg} / \mathrm{L}\end{array}$ & $65.1-118 \%$ & [283] \\
\hline
\end{tabular}


Table 3. Cont.

\begin{tabular}{|c|c|c|c|c|c|c|c|c|c|c|c|c|c|}
\hline \multicolumn{8}{|c|}{ Extraction conditions } & \multicolumn{3}{|c|}{$\begin{array}{l}\text { Desorption } \\
\text { conditions }\end{array}$} & \multirow[b]{2}{*}{ LOD } & \multirow[b]{2}{*}{ Recovery } & \multirow[b]{2}{*}{ Ref. } \\
\hline $\begin{array}{l}\text { Food } \\
\text { Sample } \\
\end{array}$ & Analyte & Technique & Fiber & $\begin{array}{l}\text { Time } \\
\text { [min] }\end{array}$ & $\begin{array}{l}\text { Temp. } \\
{\left[{ }^{\circ} \mathrm{C}\right]}\end{array}$ & $\begin{array}{l}\text { Salt } \\
\text { addition }\end{array}$ & Deriv. & $\begin{array}{l}\text { Time } \\
\text { [min] }\end{array}$ & $\begin{array}{l}\text { Temp. } \\
{\left[{ }^{\circ} \mathrm{C}\right]}\end{array}$ & Detection & & & \\
\hline \multicolumn{14}{|c|}{ Volatlile toxic compounds } \\
\hline \multicolumn{14}{|c|}{ Contaminants } \\
\hline $\begin{array}{l}\text { Drinking } \\
\text { water }\end{array}$ & $\begin{array}{l}\text { organic micro- } \\
\text { pollutants }\end{array}$ & DI-SPME & PDMS-DVB & 30 & 60 & - & - & - & 280 & GC-MS & $\begin{array}{l}0.5-10 \\
\mu \mathrm{g} / \mathrm{L}\end{array}$ & - & [284] \\
\hline $\begin{array}{l}\text { Apple, } \\
\text { apple juice, } \\
\text { tomato }\end{array}$ & $\begin{array}{l}\text { organophosphorus } \\
\text { pesticides }\end{array}$ & HS-SPME & $\begin{array}{l}\text { PDMS-DVB } \\
\text { including B15C5 } \\
\text { prepared by sol-gel } \\
\text { technology }\end{array}$ & 45 & 70 & $5 \mathrm{~g} \mathrm{NaCl}$ & - & 5 & 270 & GC-FPD & $\begin{array}{l}0.003- \\
0.09 \mathrm{ng} / \mathrm{g}\end{array}$ & $\begin{array}{l}\text { apple } \\
\text { juice: } 71.5 \\
-01.6 \% \\
\text { apple: } \\
83.3-97.7 \% \\
\text { tomato: } \\
55.3- \\
105.3 \% \\
(\text { spiked } \\
5 \mathrm{ng} / \mathrm{g})\end{array}$ & [285] \\
\hline Apples & $\begin{array}{l}\text { polycyclic aromatic } \\
\text { hydrocarbons, } \\
\text { benzene, toluene, } \\
\text { ethylbenzene, xylene }\end{array}$ & HS-SPME & CAR-PDMS & 45 & 60 & - & - & 5 & 250 & GC-MS & $\begin{array}{l}0.02 \mathrm{mg}< \\
\mathrm{LOD}< \\
0.26 \mathrm{mg}\end{array}$ & $\begin{array}{l}0.012- \\
0.140 \mu \mathrm{g}\end{array}$ & [286] \\
\hline $\begin{array}{l}\text { Fruit and } \\
\text { vegetables }\end{array}$ & pesticide residues & HS-SPME & PDMS & 34 & 62 & $10 \% \mathrm{NaCl}$ & - & 7 & 270 & GC-MS & $\begin{array}{l}0.35-8.33 \\
\mu \mathrm{g} / \mathrm{kg}\end{array}$ & $73-118 \%$ & [287] \\
\hline $\begin{array}{l}\text { Packaged } \\
\text { fresh } \\
\text { vegetables }\end{array}$ & $\begin{array}{l}\text { volatiles derived from } \\
\text { Salmonella } \\
\text { typhimurium }\end{array}$ & HS-SPME & CAR-PDMS & 15 & 20 & - & - & 3 & - & GC-MS & - & - & [288] \\
\hline
\end{tabular}


Table 3. Cont.

\begin{tabular}{|c|c|c|c|c|c|c|c|c|c|c|c|c|c|}
\hline \multicolumn{8}{|c|}{ Extraction conditions } & \multicolumn{3}{|c|}{$\begin{array}{l}\text { Desorption } \\
\text { conditions } \\
\end{array}$} & \multirow[b]{2}{*}{ LOD } & \multirow[b]{2}{*}{ Recovery } & \multirow[b]{2}{*}{ Ref. } \\
\hline $\begin{array}{l}\text { Food } \\
\text { Sample } \\
\end{array}$ & Analyte & Technique & Fiber & $\begin{array}{l}\text { Time } \\
\text { [min] }\end{array}$ & $\begin{array}{l}\text { Temp. } \\
{\left[{ }^{\circ} \mathrm{C}\right]}\end{array}$ & $\begin{array}{l}\text { Salt } \\
\text { addition }\end{array}$ & Deriv. & $\begin{array}{l}\text { Time } \\
\text { [min] }\end{array}$ & $\begin{array}{l}\text { Temp. } \\
{\left[{ }^{\circ} \mathrm{C}\right]}\end{array}$ & Detection & & & \\
\hline \multicolumn{14}{|c|}{ Volatlile toxic compounds } \\
\hline \multicolumn{14}{|c|}{ Contaminants } \\
\hline Vegetables & PAHs & HS-SPME & $\begin{array}{l}\text { benzoxy-C[6]/OH- } \\
\text { TSO prepared by sol- } \\
\text { gel technology }\end{array}$ & 40 & 60 & $\mathrm{NaCl}$ & - & 10 & 280 & GC-FID & $\begin{array}{l}0.04-2.32 \\
\mathrm{ng} / \mathrm{g}\end{array}$ & $\begin{array}{l}81.07- \\
107.5 \%\end{array}$ & [289] \\
\hline Vegetables & $\begin{array}{l}\text { organophosphorus } \\
\text { pesticide residues }\end{array}$ & DI-SPME & PA & 30 & RT & $10 \% \mathrm{NaCl}$ & - & 11 & 260 & GC-FPD & $\begin{array}{l}0.01-0.14 \\
\mu \mathrm{g} / \mathrm{L}\end{array}$ & - & [290] \\
\hline Radish & $\begin{array}{l}\text { organochlorine } \\
\text { pesticides }\end{array}$ & HS-SPME & $\begin{array}{l}\text { calix[4]arene/hy- } \\
\text { droxy-terminated } \\
\text { silicone oil prepared } \\
\text { by sol-gel technology }\end{array}$ & 30 & 70 & $\begin{array}{l}1.0 \mathrm{~g} \\
\mathrm{~K}_{2} \mathrm{SO}_{4}\end{array}$ & - & 2 & 270 & GC-ECD & $\begin{array}{l}1.27-174 \\
\mathrm{ng} / \mathrm{kg}\end{array}$ & $\begin{array}{l}83.05- \\
119.3 \%\end{array}$ & [291] \\
\hline $\begin{array}{l}\text { Roasted } \\
\text { coffee }\end{array}$ & furan & HS-SPME & CAR-PDMS & 30 & 35 & - & - & - & - & GC-MS & $3-10 \mu \mathrm{g} / \mathrm{kg}$ & $76-101 \%$ & [292] \\
\hline Wine & $\begin{array}{l}\text { 2,4,6-trichloroanisole, } \\
\text { dibutyl phthalate }\end{array}$ & SR-SPME & $\begin{array}{l}\text { graphene and } \\
\text { graphene oxide } \\
\text { prepared by sol-gel } \\
\text { technology }\end{array}$ & 20 & 45 & $20 \% \mathrm{NaCl}$ & - & 5 & 250 & GC-MS & $0.3 \mathrm{ng} / \mathrm{L}$ & $\begin{array}{l}96.96 \% / \\
98.20 \%\end{array}$ & [293] \\
\hline $\begin{array}{l}\text { Still and } \\
\text { fortified } \\
\text { wines }\end{array}$ & $\begin{array}{l}\text { fungicides captan, } \\
\text { chlorthalonil, folpet, } \\
\text { iprodione, } \\
\text { procymidone and } \\
\text { vinclozolin, acaricide } \\
\text { dicofol }\end{array}$ & DI-SPME & PDMS & 60 & 35 & - & - & 3 & 250 & $\begin{array}{l}\text { GC- } \\
\text { MS/MS }\end{array}$ & - & $70-120 \%$ & [294] \\
\hline
\end{tabular}


Table 3. Cont.

\begin{tabular}{|c|c|c|c|c|c|c|c|c|c|c|c|c|c|}
\hline \multirow[b]{2}{*}{$\begin{array}{l}\text { Food } \\
\text { Sample }\end{array}$} & \multirow[b]{2}{*}{ Analyte } & \multicolumn{6}{|c|}{ Extraction conditions } & \multicolumn{3}{|c|}{$\begin{array}{l}\text { Desorption } \\
\text { conditions }\end{array}$} & \multirow[b]{2}{*}{ LOD } & \multirow[b]{2}{*}{ Recovery } & \multirow[b]{2}{*}{ Ref. } \\
\hline & & Technique & Fiber & $\begin{array}{l}\text { Time } \\
\text { [min] }\end{array}$ & $\begin{array}{l}\text { Temp. } \\
{\left[{ }^{\circ} \mathrm{C}\right]}\end{array}$ & $\begin{array}{l}\text { Salt } \\
\text { addition }\end{array}$ & Deriv. & $\begin{array}{l}\text { Time } \\
\text { [min] }\end{array}$ & $\begin{array}{l}\text { Temp. } \\
{\left[{ }^{\circ} \mathrm{C}\right]}\end{array}$ & Detection & & & \\
\hline \multicolumn{14}{|c|}{ Volatlile toxic compounds } \\
\hline \multicolumn{14}{|c|}{ Contaminants } \\
\hline $\begin{array}{l}\text { Breaded } \\
\text { fish } \\
\text { products }\end{array}$ & furanic compounds & HS-SPME & CAR-PDMS & 40 & $37 \pm 1$ & $3 \mathrm{~g} \mathrm{NaCl}$ & - & 10 & 280 & GC-MS & - & - & {$[295]$} \\
\hline $\begin{array}{l}\text { Fresh, deep } \\
\text { frozen, } \\
\text { canned, } \\
\text { boiled, } \\
\text { roasted fish }\end{array}$ & formaldehyde & HS-SPME & CAR-PDMS & 30 & 80 & - & $\begin{array}{l}\text { PFBH } \\
\text { A }\end{array}$ & 3 & 310 & GC-MS & $17 \mu \mathrm{g} / \mathrm{kg}$ & $94.8 \pm 1.7 \%$ & {$[81]$} \\
\hline $\begin{array}{l}\text { Cooked, } \\
\text { peeled } \\
\text { tropical } \\
\text { shrimps }\end{array}$ & $\begin{array}{l}\text { 3-methyl-1-butanal, } \\
\text { 2,3-butanedione, } \\
\text { 2-methyl-1-butanal, } \\
\text { 2,3-heptanedione and } \\
\text { trimethylamine } \\
\text { induced by isolated } \\
\text { bacteria }\end{array}$ & HS-SPME & CAR-PDMS & 25 & 40 & - & - & 5 & 280 & GC-MS & - & - & [296] \\
\hline $\begin{array}{l}\text { Packaged, } \\
\text { aged, fresh } \\
\text { beef }\end{array}$ & $\begin{array}{l}\text { VOCs associated with } \\
\text { Salmonella }\end{array}$ & HS-SPME & CAR-PDMS & 30 & $23 \pm 2^{\circ} \mathrm{C}$ & - & - & 10 & 270 & GC-MS & - & - & {$[297]$} \\
\hline
\end{tabular}


Table 3. Cont.

\begin{tabular}{|c|c|c|c|c|c|c|c|c|c|c|c|c|c|}
\hline \multicolumn{8}{|c|}{ Extraction conditions } & \multicolumn{3}{|c|}{$\begin{array}{c}\text { Desorption } \\
\text { conditions } \\
\end{array}$} & \multirow[b]{2}{*}{ LOD } & \multirow[b]{2}{*}{ Recovery } & \multirow[b]{2}{*}{ Ref. } \\
\hline $\begin{array}{l}\text { Food } \\
\text { Sample } \\
\end{array}$ & Analyte & Technique & Fiber & $\begin{array}{l}\text { Time } \\
\text { [min] }\end{array}$ & $\begin{array}{l}\text { Temp. } \\
{\left[{ }^{\circ} \mathrm{C}\right]}\end{array}$ & $\begin{array}{l}\text { Salt } \\
\text { addition }\end{array}$ & Deriv. & $\begin{array}{l}\text { Time } \\
\text { [min] }\end{array}$ & $\begin{array}{l}\text { Temp. } \\
{\left[{ }^{\circ} \mathrm{C}\right]}\end{array}$ & Detection & & & \\
\hline \multicolumn{14}{|c|}{ Volatlile toxic compounds } \\
\hline \multicolumn{14}{|c|}{ Contaminants } \\
\hline $\begin{array}{l}\text { Smoked } \\
\text { meat } \\
\text { products }\end{array}$ & PAHs & DI-SPME & PDMS & 60 & 25 & - & - & 30 & 250 & GC-MS & $\begin{array}{l}0.008- \\
0.138 \\
\mathrm{ng} / \mathrm{mL}\end{array}$ & - & {$[298]$} \\
\hline $\begin{array}{l}\text { Meat } \\
\text { roasted in } \\
\text { plastic bags }\end{array}$ & $\begin{array}{l}\text { plasticisers } \\
\text { (phthalates) }\end{array}$ & $\begin{array}{l}\text { DI-CF- } \\
\text { SPME }\end{array}$ & PA & 30 & 45 & - & - & 0.02 & 250 & GC-MS & $\begin{array}{l}0.01-0.18 \\
\mu \mathrm{g} / \mathrm{kg}\end{array}$ & - & {$[134]$} \\
\hline $\begin{array}{l}\text { Fruit } \\
\text { leathers }\end{array}$ & $\begin{array}{l}\text { carbonyl compounds } \\
\text { generated from ozone- } \\
\text { based food colorants } \\
\text { decomposition }\end{array}$ & HS-SPME & PDMS-DVB & 15 & 60 & - & $\begin{array}{l}\text { PFBHA } \\
\text { methanol }\end{array}$ & - & 250 & GC-MS & $\begin{array}{l}0.016- \\
0.030 \mu \mathrm{g} / \mathrm{L}\end{array}$ & - & {$[86]$} \\
\hline
\end{tabular}

CF: cold fiber; CNT: carbon nanotube; CSCB: Chinese traditional smoke-cured bacon; DAD: diode array detector; deriv.: derivatization; DI: direct immersion; DPrDS: dipropyl disulphide; ECD: electron capture detector; EDMA: ethylene dimethacrylate; FASST: Fast Automated Scan/SIM Type; FID: flame ionization detector; FPD: flame photometric detector; GC-O: gas chromatographyolfactometry; GMA: glycidyl methacrylate; GS: gas sensor; IMS: ion mobility spectrometry; LOD: limit of detection; LVI: large volume injection; MAED: methacrylic acid-co-ethylene dimethacrylate; MASE: membrane assisted solvent extraction; MEMF: methacrylic acid-co-ethylene dimethacrylate monolithic fiber; MHS: multiple headspace; MMF: multiple monolithic fiber; MW: multi-walled; NCI: negative chemical ionization; OH-TSO: hydroxyl-terminated silicone oil; OL: oak lactone; PAHs: polycyclic aromatic hydrocarbons; PDO: protected designation of origin; PEG: polyethylene glycol; PFBHA: pentafluorobenzyl-hydroxylamine hydrochloride; pFPD: pulsed flame photometric detector; PTR: Proton-transfer-reaction; PVC: polyvinyl chloride; QIT: quadrupole ion trap; qMS: quadrupole mass spectrometry; QTOF: quadrupole time-of-flight; ref.: reference; RT: room temperature; SAW: surface acoustic wave; SIM: selected ion monitoring; SR: space-resolved; TD: thermal desorption; temp.: temperature; TMSPMA: 3-(trimethoxysylil)propyl methacrylate; TOF: time-of-flight; TPR: template resin; VVC: various volatile compounds. 


\subsection{Aroma}

The verification of taste and fragrance attributed to specific foods has become an important part of consumer demand. For this purpose SPME methods can serve as a valuable tool to examine the presence or absence of desired aroma compounds in food products [65]. SPME has gained increasing popularity for aroma analysis in recent years (see Figure 8). A collection of recently published studies in the field of applications of SPME in food, non-food, and fragrance analysis, etc. are listed in the Handbook of Solid Phase Microextraction [75].

Various food components like proteins, lipids, and sugars affect the chemical composition found in the headspace above food items. Page and Lacroix (1993) reported that the presence of a large quantity of lipid material in food products led to a lower extraction of volatiles from the headspace [299]. To prevent this decrease it is recommended to carefully raise the extraction temperature that will increase the number of volatile constituents present in the headspace. Thereby the number of volatile constituents present in the headspace increase. Raw meat for example had no strong aroma [300], but cooking increased meat aroma [300]. Xie et al. (2008) studied compounds that have a real impact on meat aroma. Volatiles of the roasted Mini-pig pork were determined by both SPME and SDE combined with GC-MS. Additionally GC-O was applied to identify potent contributors to the special meat aroma. A total of 86 different volatile compounds have been identified, whereas only 45 odor active regions could be recognized by olfactometry. Aldehydes from lipid oxidation followed by spice components were among the highest contributors to the volatile chemical profile [205].

Another study on meat aroma was performed with Chinese traditional smoke-cured bacon (CSCB) by Yu et al. [206]. The aim of the study was to work out the substances responsible for the characteristic aroma of CSCB. In total, 48 volatile compounds (alkanes, aldehydes, ketones, alcohols, thiolethers and thiols, furans and phenol compounds) were identified and quantified using SPME coupled to GC-MS [206]. Chemical processes like smoking, oxidation and the Maillard reaction (reaction between reducing sugars and amino acids, usually under the influence of heat) are largely responsible for the occurrence of these compounds.

Another approach in aroma analysis is to verify the origin and authenticity of foods. For example, Majcher et al. [202] developed a SPME method in combination with chemometrics for the determination of volatile compounds derived from traditional Polish cheese Oscypek and its imitations [202]. Sostaric et al. (2000) used SPME-GC-MS to differentiate between natural vanilla extracts and natureidentical and synthetic vanilla flavorings [301].

The extraction of aromatic volatile compounds from alcoholic and non-alcoholic beverages by SPME was recently extensively investigated (see table 3). Câmara et al. [212] investigated aroma compounds in whisky samples. Classical analytical methods such as LLE, simultaneous extraction or distillation show relatively low reproducibility or possible contamination with solvents. Further the lengh of time required for the analysis and the selectivity of the results are such a few drawbacks, which can be overcome selectivity can be overcome by dynamic headspace SPME and gas chromatography coupled to GC-MS. In this study the use of five different SPME fibers PDMS, PA, CAR-PDMS, CW-DVB and CAR-PDMS-DVB were compared. Optimized results were achieved by saturating the samples with salt solutions and using a CAR-PDMS fiber. More than seventy compounds including ethylesters, long-chain alcohols, isoamyl acetates and fatty acids were identified [212]. 
Ong et al. [231] investigated volatile compounds derived from jackfruit applying SPME and gas chromatography-time-of-flight mass spectrometry (GCTOF-MS). In five jackfruit cultivars thirty-seven compounds were identified. Compounds responsible for the sweet and fruity aroma in jackfruit were identified as ethyl isovalerate, 3-methylbutyl acetate, 1-butanol, propyl isovalerate, isobutyl isovalerate, 2-methylbutanol, and butyl isovalerate [231].

Fruit origin and authenticity (species idenfication) can also be successfully investigated by means of SPME analysis of volatile compounds [229]. For example, the typical sensory expectations of apricot fruits are sweetness and juiciness, which are strongly related to the ripening stage apricots at harvest [302]. Aroma compounds of apricots from different regions of the world were extensively studied [303]. Major identified aroma compounds were ethyl acetate, hexyl acetate, limonene, 6-methyl-5-hepten-2-one, menthone, E-hexen-2-al, linalool, beta-ionone and cyclo-decalactone [228].

Recently the European Union established the research program Horizon 2020 to develop a validated analytical method for determination of volatiles resulting from virgin olive oils by means of SPMEGC/MS. Romero et al. [244] published a validated method including calibration curves for 29 volatile compounds. The analytical precision of $67 \%$ of these compounds had a relative standard deviation lower than $10 \%$ and accuracies were determined for $97 \%$ of the analyzed volatile compounds. The limits of detection ranged from 0.1 to $2.54 \mathrm{mg} / \mathrm{kg}$ for determined volatile compounds [244].

\subsection{Off-Flavors}

As described in Section 4.1, aroma belongs to the most important sensory characteristics of food. Food products with an inappropriate aroma impression are often rejected by consumers and may lead to a loss of consumer confidence [304]. The awareness of off-flavors in food relies mainly on their concentration occurring in the food matrix and odor threshold values. Off-flavors are defined as unpleasant odors or flavors transmitted to food through internal impairing changes [304]. Many volatile compounds are associated with unpleasant odor notes such, e.g., putrid, musty, rotten, skunk, etc. However, the received off-flavor impression often depends on the concentration of the related aroma compounds. The most common off-flavor compounds are those that cause musty, earthy off-flavors (haloanisoles, halophenols, geosmin and methylisoborneol) and medicinal off-flavors (phenolic compounds, sulfur-containing compounds and carbonyl compounds) [304]. Other off-flavor compounds are generally classified according to their origin, including microbially derived off-flavors, compounds originating from packaging materials, from cleaning agents, and compounds resulting from Maillard reactions [304].

The impact of the cork composition on the sensory properties of wines was investigated by means of SPME GC-MS [272]. Cork was shown to have an active role in the sorption of volatile phenols from wine. In particular, the sorption properties of 4-ethylphenol and 4-ethylguaiacol phenols in hydro-alcoholic medium were investigated by Gallardo-Chacón and Karbowiak [272]. Through the high sorption activity of corks the concentration of 4-ethylphenol and 4-ethylguaiacol was decreased. High concentration of these compounds can lead to the formation of off-flavor. Both, in models and real wine samples the tested cork leads to a significant reduction of the mentioned off-flavor compounds in wine [272].

The oxidative stability of conventional high-oleic sunflower oil and analytical and sensory lipid oxidation parameters in conventional and high-oleic rapeseed oil were reported by Petersen et al. [260,261]. 
It was concluded that the combination of volatile compound analysis with HS-SPME-GC and multivariate statistical methods provides a sensitive tool in differentiating conventional sunflower oil and high-oleic sunflower oil as by means of volatile lipid oxidation marker compounds. The same applies to differentiating rapeseed oil and high-oleic rapeseed oil.

\subsection{Volatile Toxic Compounds}

Various sources of microbiological and chemical hazardous compounds may occur in foods. The latter may include natural toxicants, such as mycotoxins [305-307], marine toxins [308], and environmental contaminants, such as mercury and lead [309-314].

Among these substances for example volatile toxic aldehydes like formaldehyde has recently received increased attention. Formaldehyde is formed post-mortem by the enzymatic reduction of trimethylamine-N-oxide to formaldehyde and dimethylamine [315,316]. Due to its high volatility, formaldehyde is captured well by SPME for subsequent measurement. Firstly, in 2005 Bianchi et al. [317] developed a SPME method with in situ derivatisation with pentafluorobenzyl-hydroxylamine hydrochloride (PFBHA). In 2007, Bianchi et al. [81] used a SPME-GC-MS method based on the same derivatization to evaluate the formaldehyde content of various fish products.

Additional non-flavor contaminants are polycyclic aromatic hydrocarbons (PAHs), identified as pollutants in the environment and food items [1-3]. Bianchi et al. [276] developed a SPME method with a coating based on the use of diethoxydiphenylsilane produced by sol-gel technology for determination of PAHs at trace levels in milk. For vegetables, a simple, sensitive and affordable method has been developed for the quantitation of eight PAHs by Lei et al. [289]. The method was based on HS-SPME connected with GC-FID. The sol-gel designed benzoxy-C[6]/OH-TSO proved to have a good capability to capture aromatic compounds such as phthalate acid esters. Compared to the tested PDMS fiber and $\mathrm{C}[4] / \mathrm{OH}-\mathrm{TSO}$ fiber, the benzoxy-C[6]/OH-TSO showed to have the highest affinity to PAHs [289].

The VOCs 2-nitrophenol and 3-fluoroaniline were extracted, separated and detected by headspacesolid phase microextraction coupled to gas chromatography-mass spectrometry (HS-SPME-GC-MS). It was stated to be a potential rapid method for future development [275].

Microorganisms can also be responsible for food contamination and spoilage. The metabolic activity of microorganisms through breakdown of compounds in food leads to the release of volatile organic compounds [41]. Extensive research was carried out in order to identify microbial activities in foods using SPME [275,288,297,318]. Siripatrawan and Harte [288] investigated the occurrence of Salmonella typhimurium present in packaged alfalfa sprouts. For that purpose CAR/PDMS fibers were compared to PDMS/DVB fibers which extracted lesser sulfur volatiles. Sulfur-containing compounds seem to be a major marker compound for spoiled food stuffs, off-flavor in milk, and the presence of pathogens [288]. Other volatile organic compounds such as 2-nitrophenol and 3-fluoroaniline may be associated with the presence of Listeria monocytogenes contamination in food [318].

Pesticides are not only contaminants, but are also non-flavor compounds occurring in food items. The simultaneous determination of fourteen multiclass pesticide residues in fruit and vegetable samples by means of HS-SPME coupled to GC-MS were reported by Abdulra'uf and Tan [287]. The method development was based on multivariate experimental designs (Plancket-Burman and central composite design) conducted in two stages. Internal standard calibration was applied for quantitation (detection 
limits between 0.35 and $8.33 \mu \mathrm{g} / \mathrm{kg}$ ). Compared to liquid-liquid extraction techniques and SPE, which are commonly used in pesticide analysis, SPME takes less time, is less labor-intensive, does not require any solvents and simplifies sample preparation to one single step and one device [41].

\section{Environmental Applications}

SPME has been widely applied to the sampling and analysis of environmental matrices including air, water, soil, and sediment samples, in on-site or off-site analysis $[20,16,18]$. Hundreds of papers reporting SPME applications in the field of environmental analysis have been published in recent years (see Table 4), the vast majority of which referred to aqueous samples (see Figure 10).

\subsection{Air Samples}

Applications of SPME in air sampling can be performed on-site [319,320] or in the laboratory after collecting air samples in suitable containers, e.g., bags [102,321], glass bulbs [181,322], or pre-evacuated vessels [323]. The analytes are extracted either by direct exposure of the fiber to the air sample or by headspace method after absorption in a suitable liquid medium [324,325] or adsorption on a solid material, e.g., Tenax TA [326]. Some authors use dynamic flux chambers for air sampling [133,327-329]. Most applications published on SPME analysis of gaseous samples involve the use of commercially available SPME fibers, although novel fiber coatings have already been developed for this purpose as well. For example, a sol-gel single-walled carbon nanotube/silica composite coated SPME has been developed for the analysis of organohalogen compounds in workplace air [133].

\section{SPME in environmental analysis between 2005 and 2015}

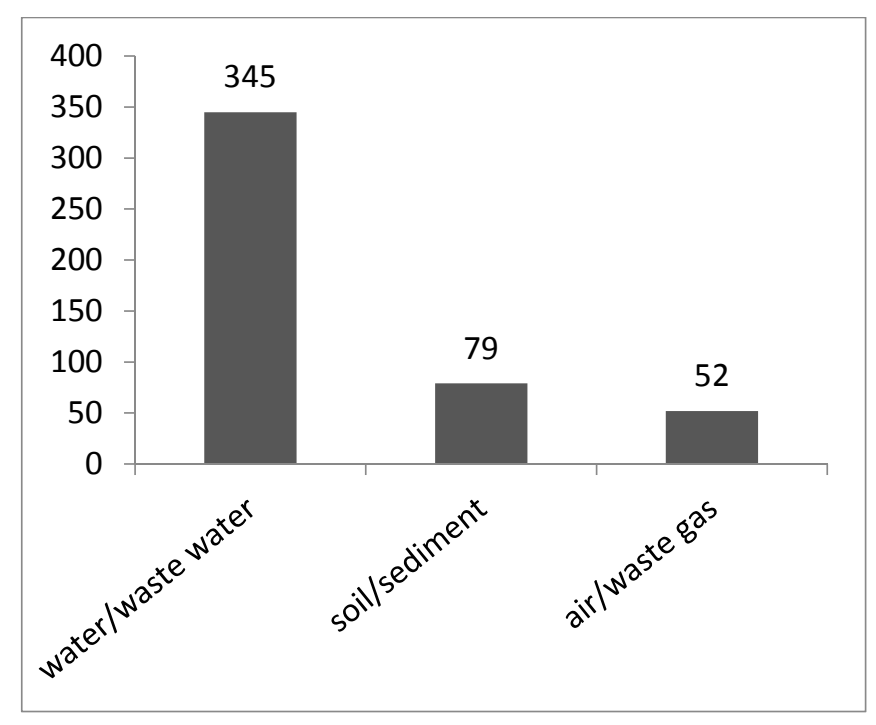

Figure 10. Applications of SPME to different environmental matrices. Number of papers based on ScienceDirect search for years 2005-2015. 
Table 4. SPME applications in environmental analysis.

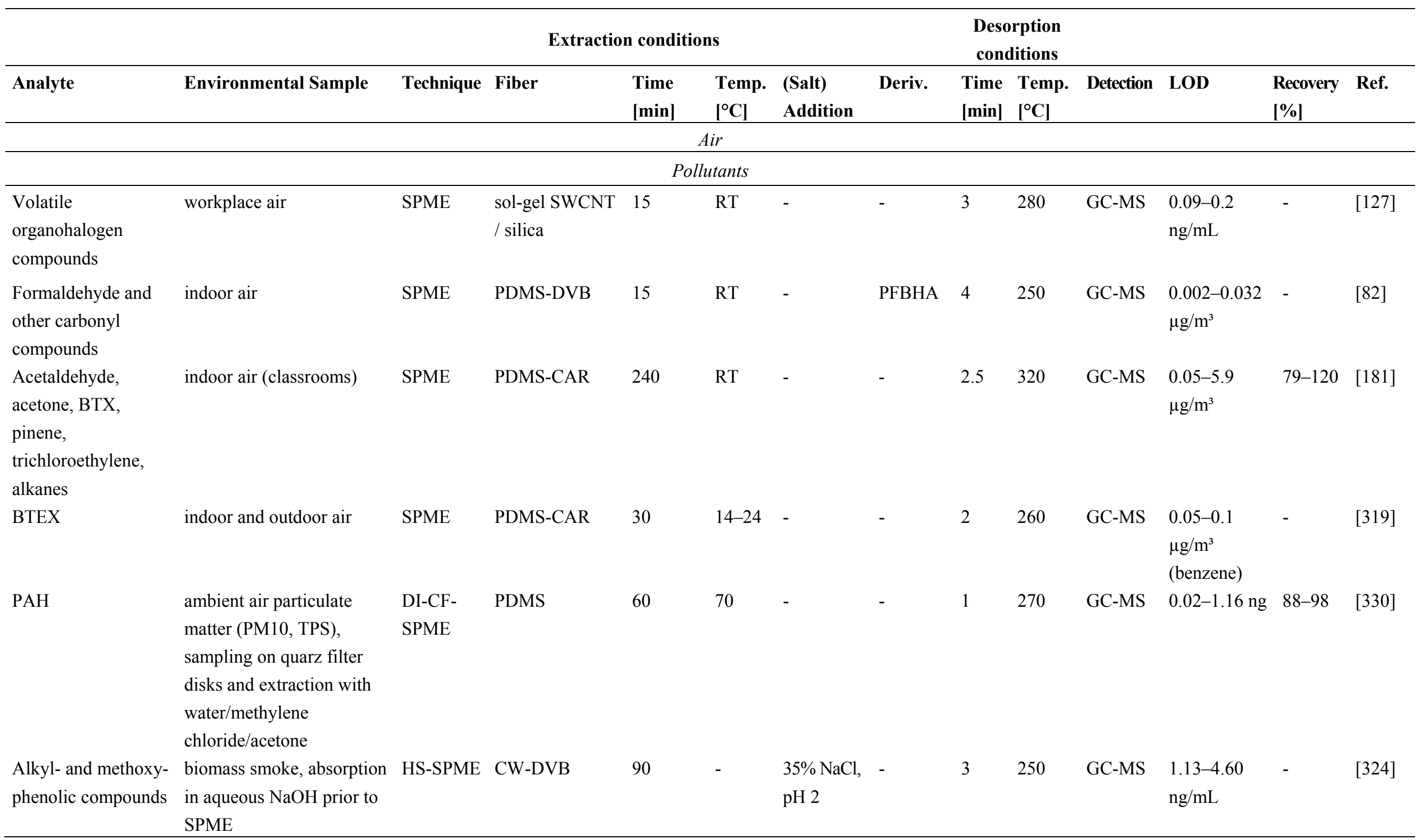


Table 4. Cont

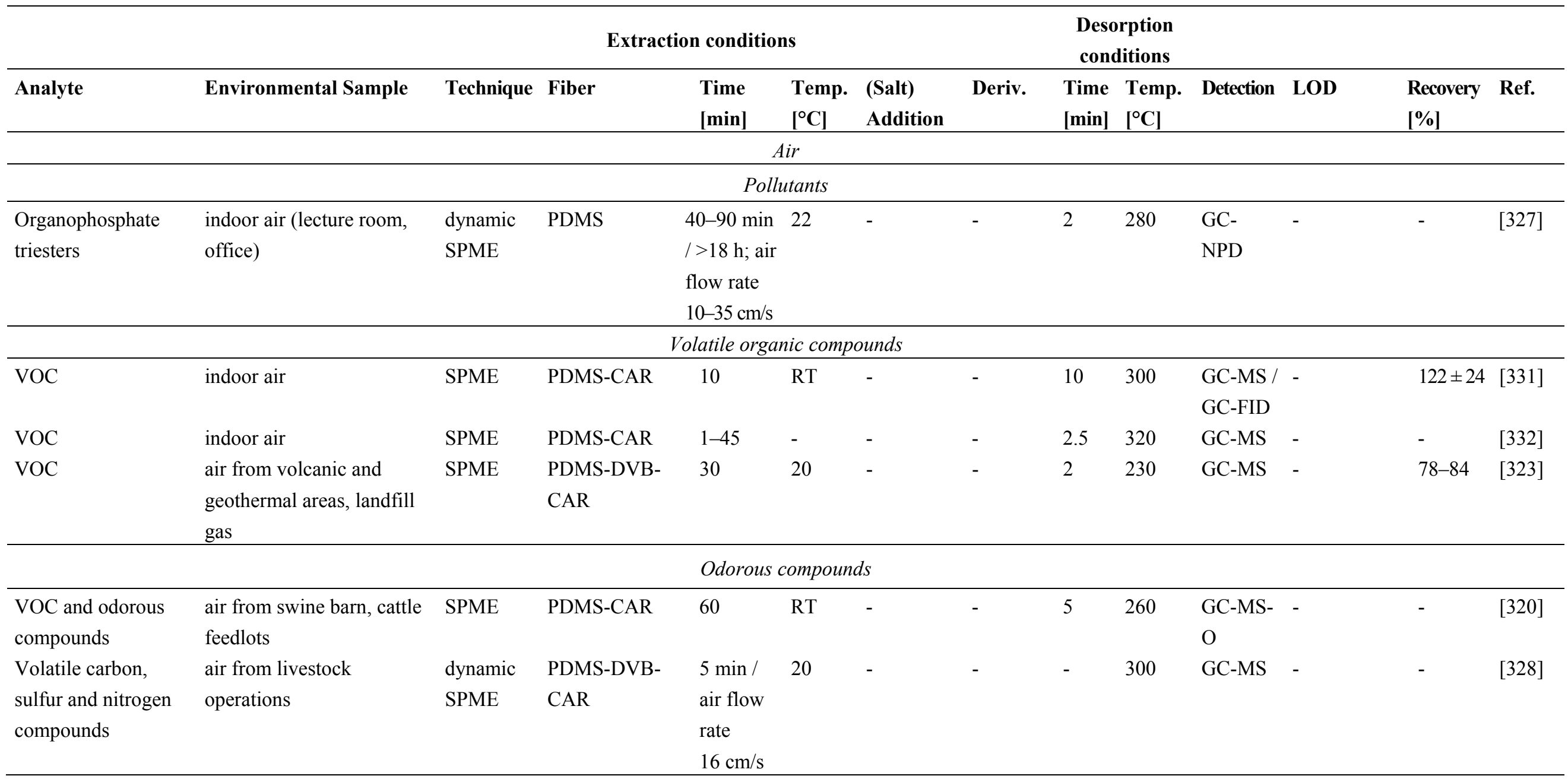


Table 4. Cont.

\begin{tabular}{|c|c|c|c|c|c|c|c|c|c|c|c|c|c|}
\hline \multirow[b]{2}{*}{ Analyte } & \multirow[b]{2}{*}{ Environmental Sample } & \multirow[b]{2}{*}{ Technique } & \multicolumn{4}{|c|}{ Extraction conditions } & \multirow[b]{2}{*}{ Deriv. } & \multicolumn{2}{|c|}{$\begin{array}{l}\text { Desorption } \\
\text { conditions }\end{array}$} & \multirow[b]{2}{*}{ Detection } & \multirow[b]{2}{*}{ LOD } & \multirow[b]{2}{*}{$\begin{array}{l}\text { Recovery } \\
{[\%]}\end{array}$} & \multirow[b]{2}{*}{ Ref. } \\
\hline & & & Fiber & $\begin{array}{l}\text { Time } \\
{[\mathrm{min}]}\end{array}$ & $\begin{array}{l}\text { Temp. } \\
{\left[{ }^{\circ} \mathrm{C}\right]}\end{array}$ & $\begin{array}{l}\text { (Salt) } \\
\text { Addition } \\
\end{array}$ & & $\begin{array}{l}\text { Time } \\
\text { [min] }\end{array}$ & $\begin{array}{l}\text { Temp. } \\
{\left[{ }^{\circ} \mathrm{C}\right]}\end{array}$ & & & & \\
\hline \multicolumn{14}{|c|}{ Odorous compounds } \\
\hline $\begin{array}{l}\text { VOC and odorous } \\
\text { compounds }\end{array}$ & $\begin{array}{l}\text { swine barn particulate } \\
\text { matter (TPS, PM10, } \\
\text { PM2.5, PM1), adsorption } \\
\text { on TEOM filters prior to } \\
\text { SPME }\end{array}$ & HS-SPME & PDMS-CAR & 180 & 25 & - & - & 40 & 260 & $\begin{array}{l}\text { GC-MS- } \\
\mathrm{O}\end{array}$ & - & - & {$[333]$} \\
\hline Odorous compounds & waste gas from fat refinery & SPME & PDMS-CAR & 30 & RT & - & - & 5 & 290 & $\begin{array}{l}\text { GC-MS / } \\
\text { GC-FID- } \\
\mathrm{O}\end{array}$ & - & - & {$[102]$} \\
\hline Odorous compounds & $\begin{array}{l}\text { gaseous effluents from } \\
\text { production of poultry } \\
\text { feather and viscera meal, } \\
\text { condensed prior to SPME }\end{array}$ & HS-SPME & $\begin{array}{l}\text { PDMS-DVB- } \\
\text { CAR }\end{array}$ & 20 & 50 & - & - & 4 & 250 & GC-MS & - & - & {$[325]$} \\
\hline $\begin{array}{l}\text { Volatile organic } \\
\text { sulfur compounds }\end{array}$ & $\begin{array}{l}\text { air from different areas of } \\
\text { sewage treatment plant }\end{array}$ & SPME & PDMS-CAR & 45 & $22 / \mathrm{RT}$ & - & - & 2 & 200 & GC-MS & $\begin{array}{l}0.01-0.08 \\
\mu \mathrm{g} / \mathrm{m}^{3}\end{array}$ & $75-96$ & {$[322]$} \\
\hline Synthetic musks & $\begin{array}{l}\text { indoor air, adsorption on } \\
\text { Tenax TA prior to SPME }\end{array}$ & HS-SPME & $\begin{array}{l}\text { DVB-CAR- } \\
\text { PDMS }\end{array}$ & 20 & 100 & $\begin{array}{l}100 \mu \mathrm{L} \\
\text { acetone }\end{array}$ & - & 5 & 270 & GC-MS & $\begin{array}{l}0.029-0.380 \\
\mathrm{ng} / \mathrm{m}^{3}\end{array}$ & $85-103$ & {$[326]$} \\
\hline Trimethylamine & ambient air & SPME & PDMS-DVB & 10 & $\begin{array}{l}22 / \\
\text { RT }\end{array}$ & - & - & 3 & 210 & GC-FID & & & {$[321]$} \\
\hline Diacetyl & air & SPME & PDMS & 2 & RT & - & - & 1 & 250 & GC-MS & $0.05 \mathrm{ppm}$ & - & {$[200]$} \\
\hline Monoterpenes & plant emissions, ambient air & SPME & PDMS-DVB & 20 & $\begin{array}{l}25 / \\
\text { RT }\end{array}$ & - & - & 5 & 250 & GC-MS & $4-20 \mathrm{ppt}$ & - & [329] \\
\hline
\end{tabular}


Table 4. Cont.

\begin{tabular}{|c|c|c|c|c|c|c|c|c|c|c|c|c|c|}
\hline & & & Extrac & n condi & & & & $\begin{array}{l}\text { Deso } \\
\text { conc }\end{array}$ & $\begin{array}{l}\text { rption } \\
\text { litions }\end{array}$ & & & & \\
\hline Analyte & Environmental Sample & Technique & Fiber & $\begin{array}{l}\text { Time } \\
{[\mathrm{min}]}\end{array}$ & $\begin{array}{l}\text { Temp. } \\
{\left[{ }^{\circ} \mathrm{C}\right]}\end{array}$ & $\begin{array}{l}\text { (Salt) } \\
\text { Addition }\end{array}$ & Deriv. & $\begin{array}{l}\text { Time } \\
\text { [min] }\end{array}$ & $\begin{array}{l}\text { Temp. } \\
{\left[{ }^{\circ} \mathrm{C}\right]}\end{array}$ & Detection & LOD & $\begin{array}{l}\text { Recovery } \\
{[\%]}\end{array}$ & Ref. \\
\hline \multicolumn{14}{|c|}{ Water } \\
\hline \multicolumn{14}{|c|}{ Odorous compounds } \\
\hline $\begin{array}{l}\text { Earthy-musty } \\
\text { odorants }\end{array}$ & $\begin{array}{l}\text { source, product, and tap } \\
\text { water from different } \\
\text { waterworks }\end{array}$ & HS-SPME & $\begin{array}{l}\text { PDMS-DVB- } \\
\text { CAR }\end{array}$ & 30 & 60 & $25 \% \mathrm{NaCl}$ & - & 5 & 230 & GC-MS & $0.1-1.3 \mathrm{ng} / \mathrm{L}$ & $83-112$ & {$[334]$} \\
\hline $\begin{array}{l}\text { Earthy-musty } \\
\text { odorants }\end{array}$ & $\begin{array}{l}\text { tap water, river water, lake } \\
\text { water }\end{array}$ & HS-SPME & $\begin{array}{l}\text { PDMS-DVB- } \\
\text { CAR }\end{array}$ & 30 & 90 & - & - & - & - & GC-MS & $\begin{array}{l}0.25-0.61 \\
\mathrm{ng} / \mathrm{L}\end{array}$ & $65-92$ & {$[335]$} \\
\hline $\begin{array}{l}\text { Earthy-musty } \\
\text { odorants }\end{array}$ & tap water, lake water & HS-SPME & $\begin{array}{l}\text { PDMS-DVB- } \\
\text { CAR }\end{array}$ & 30 & 50 & $30 \% \mathrm{NaCl}$ & - & 3 & 265 & GC-MS & $\begin{array}{l}0.32-0.66 \\
\mathrm{ng} / \mathrm{L}\end{array}$ & $86-113$ & {$[336]$} \\
\hline $\begin{array}{l}\text { Odorous } \\
\text { trichlorobromo- } \\
\text { phenols }\end{array}$ & tap water, river water & HS-SPME & $\begin{array}{l}\text { PDMS-DVB- } \\
\text { CAR }\end{array}$ & 90 & 60 & $\begin{array}{l}\mathrm{NaCl} \\
6.5 \mathrm{~g} / \\
30 \mathrm{~mL}\end{array}$ & $\begin{array}{l}\text { di- } \\
\text { methyl } \\
\text { sulfate / } \\
\mathrm{NaOH}\end{array}$ & 1 & 270 & GC-MS & $\begin{array}{l}0.22-0.95 \\
\mathrm{ng} / \mathrm{L}\end{array}$ & - & [337] \\
\hline $\begin{array}{l}\text { Algal taste and odor } \\
\text { compounds }\end{array}$ & lake water & HS-SPME & $\begin{array}{l}\text { PDMS-DVB- } \\
\text { CAR }\end{array}$ & 30 & 65 & $\mathrm{NaCl}$ & - & 3 & 250 & $\begin{array}{l}\text { GC-MS- } \\
\mathrm{O}\end{array}$ & $\begin{array}{l}\text { sub to low } \\
\text { ppt range }\end{array}$ & $80-115$ & {$[87]$} \\
\hline $\begin{array}{l}\text { Volatile sulfur } \\
\text { compounds }\end{array}$ & odorous freshwater lakes & HS-SPME & PDMS-CAR & 30 & 45 & - & - & 3 & 250 & GC-FPD & $\begin{array}{l}1.6-93.5 \\
\mathrm{ng} / \mathrm{L}\end{array}$ & $87-112$ & [338] \\
\hline $\begin{array}{l}\text { Nitro musk } \\
\text { fragrances }\end{array}$ & tap water, wastewater & HS-SPME & $\begin{array}{l}\text { PDMS-CAR, } \\
\text { PDMS-DVB }\end{array}$ & 25 & 100 & - & - & 2 & $\begin{array}{l}300 / \\
270\end{array}$ & $\begin{array}{l}\mathrm{GC}- \\
\mu \mathrm{ECD}\end{array}$ & $\begin{array}{l}0.25-3.6 \\
\mathrm{ng} / \mathrm{L}\end{array}$ & $96-108$ & [339] \\
\hline \multicolumn{14}{|c|}{ Volatile and semivolatile organic compounds } \\
\hline VOC & $\begin{array}{l}\text { surface water, wastewater } \\
\text { from wastewater treatment } \\
\text { plant and from municipal } \\
\text { solid-waste treatment plant }\end{array}$ & HS-SPME & PDMS-CAR & 30 & 50 & $10 \% \mathrm{NaCl}$ & - & 5 & 280 & $\begin{array}{l}\text { GC-MS- } \\
\text { MS }\end{array}$ & $\begin{array}{l}0.005-2 \\
\mu \mathrm{g} / \mathrm{L}\end{array}$ & $70-120$ & {$[340]$} \\
\hline
\end{tabular}


Table 4. Cont

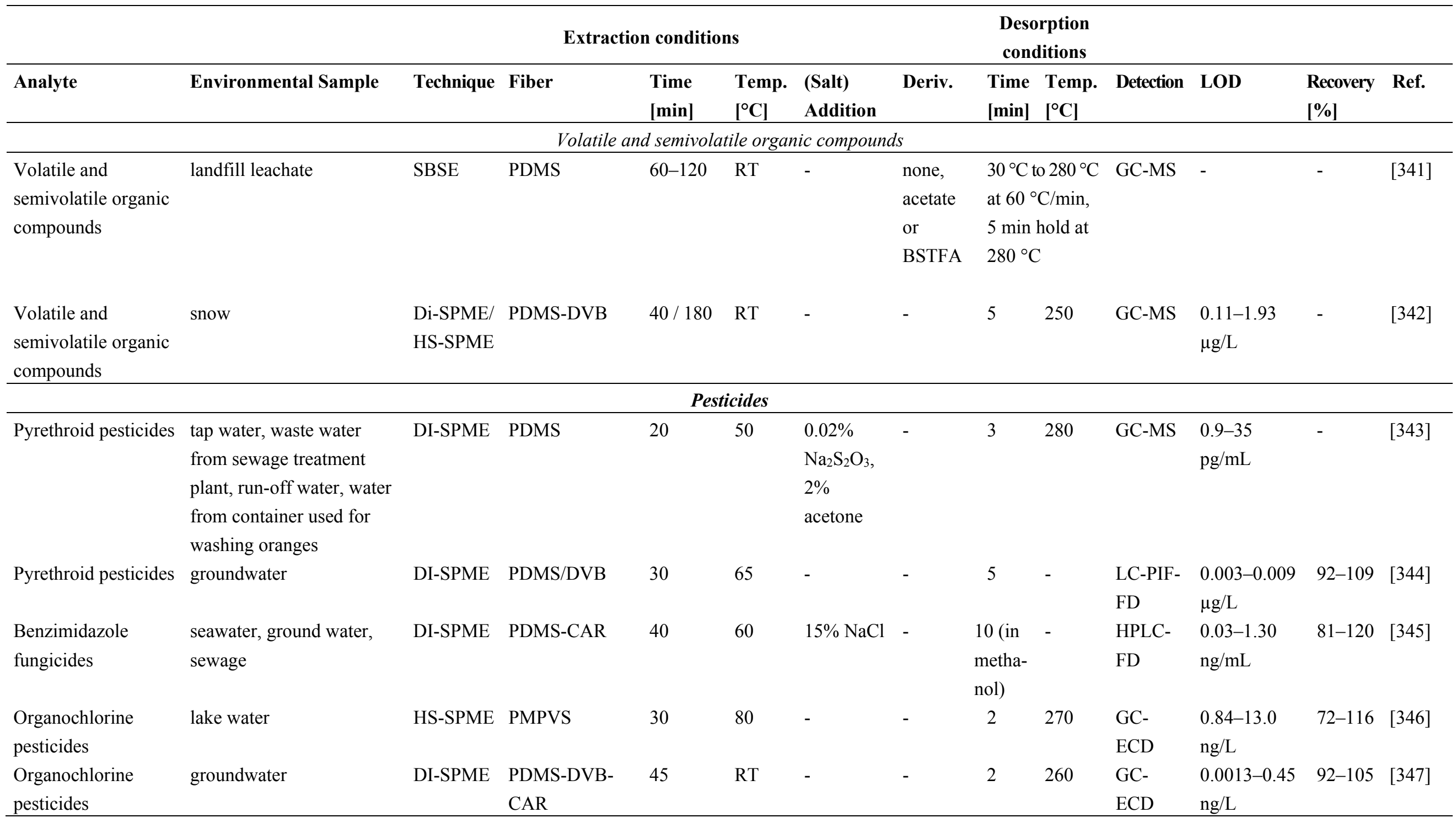


Table 4. Cont.

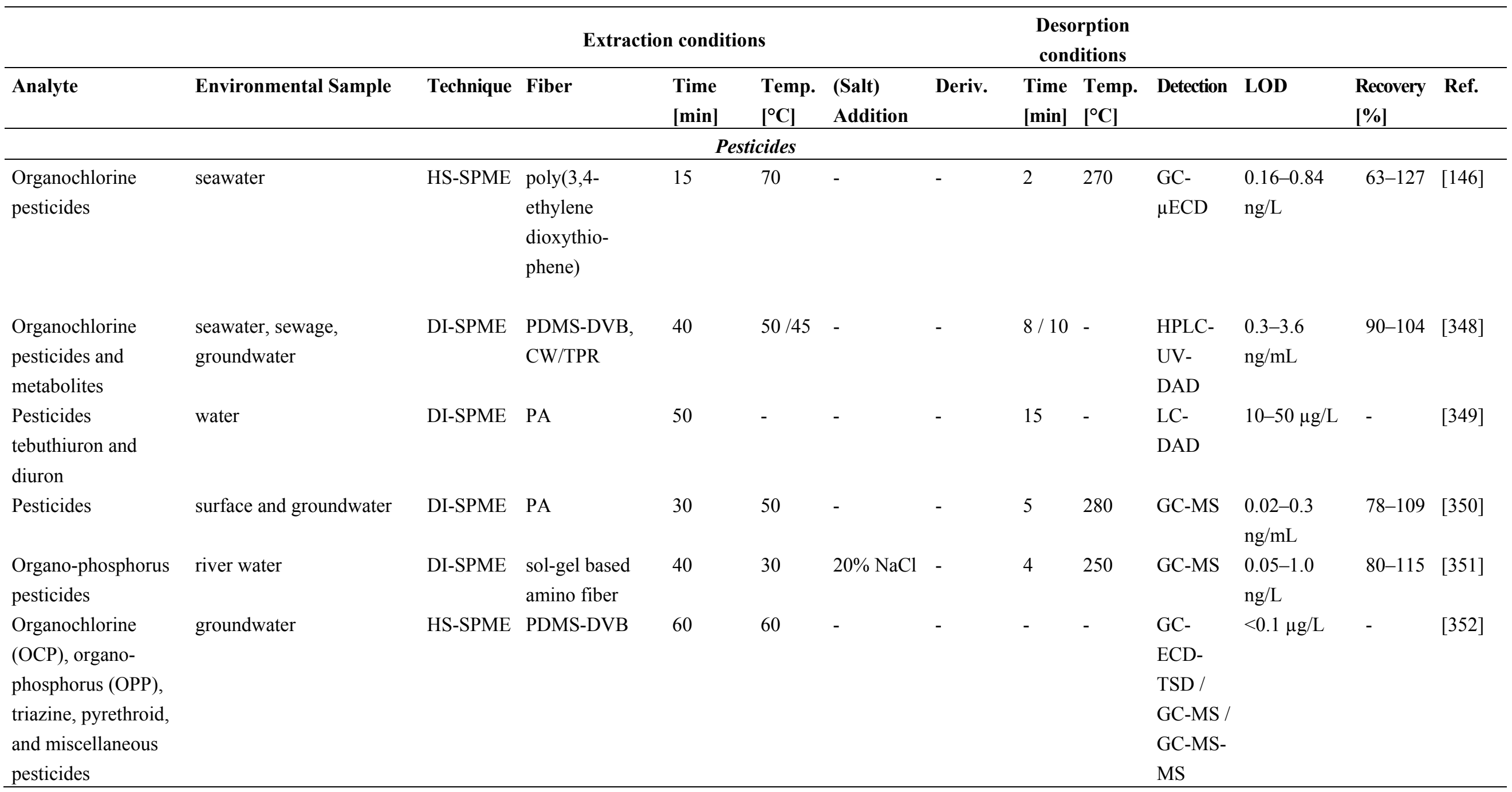


Table 4. Cont.

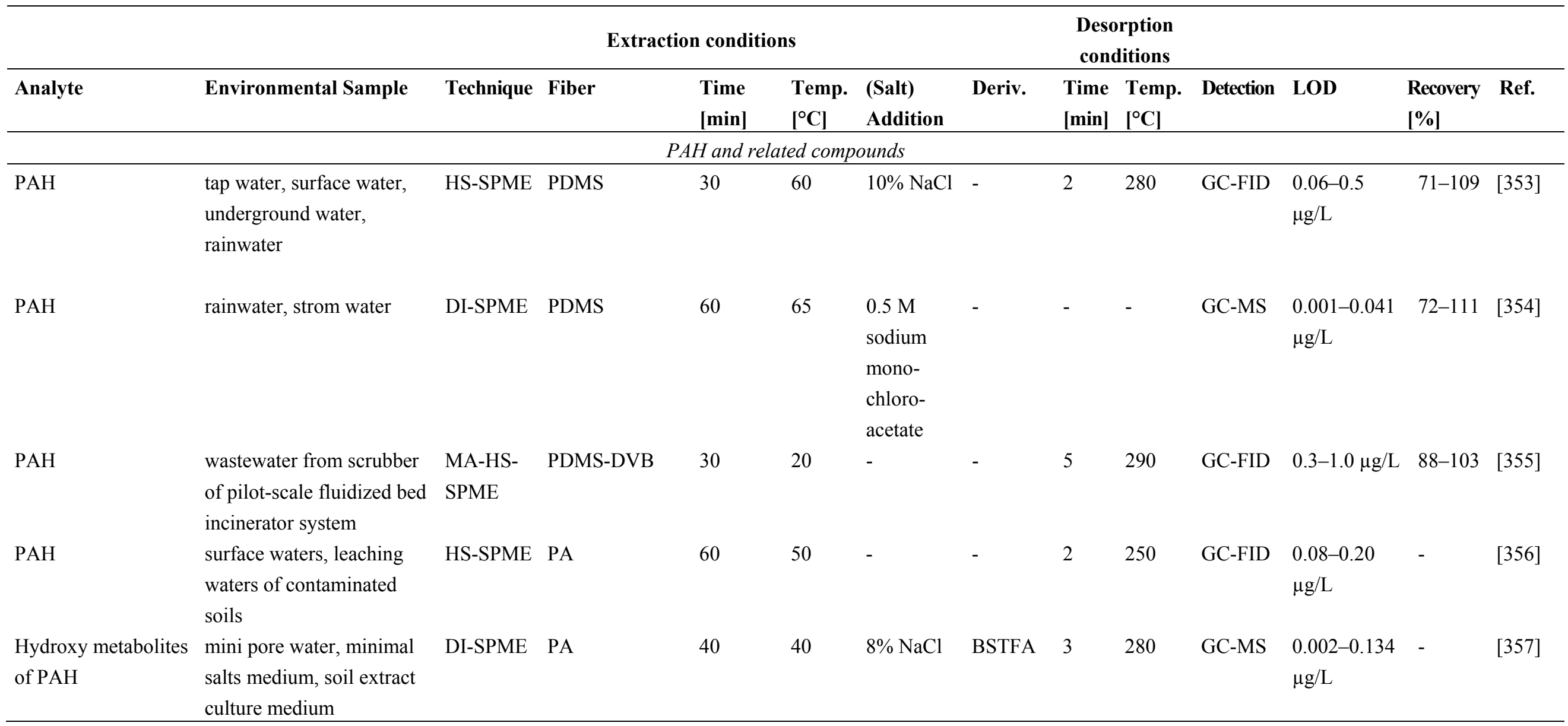


Table 4. Cont.

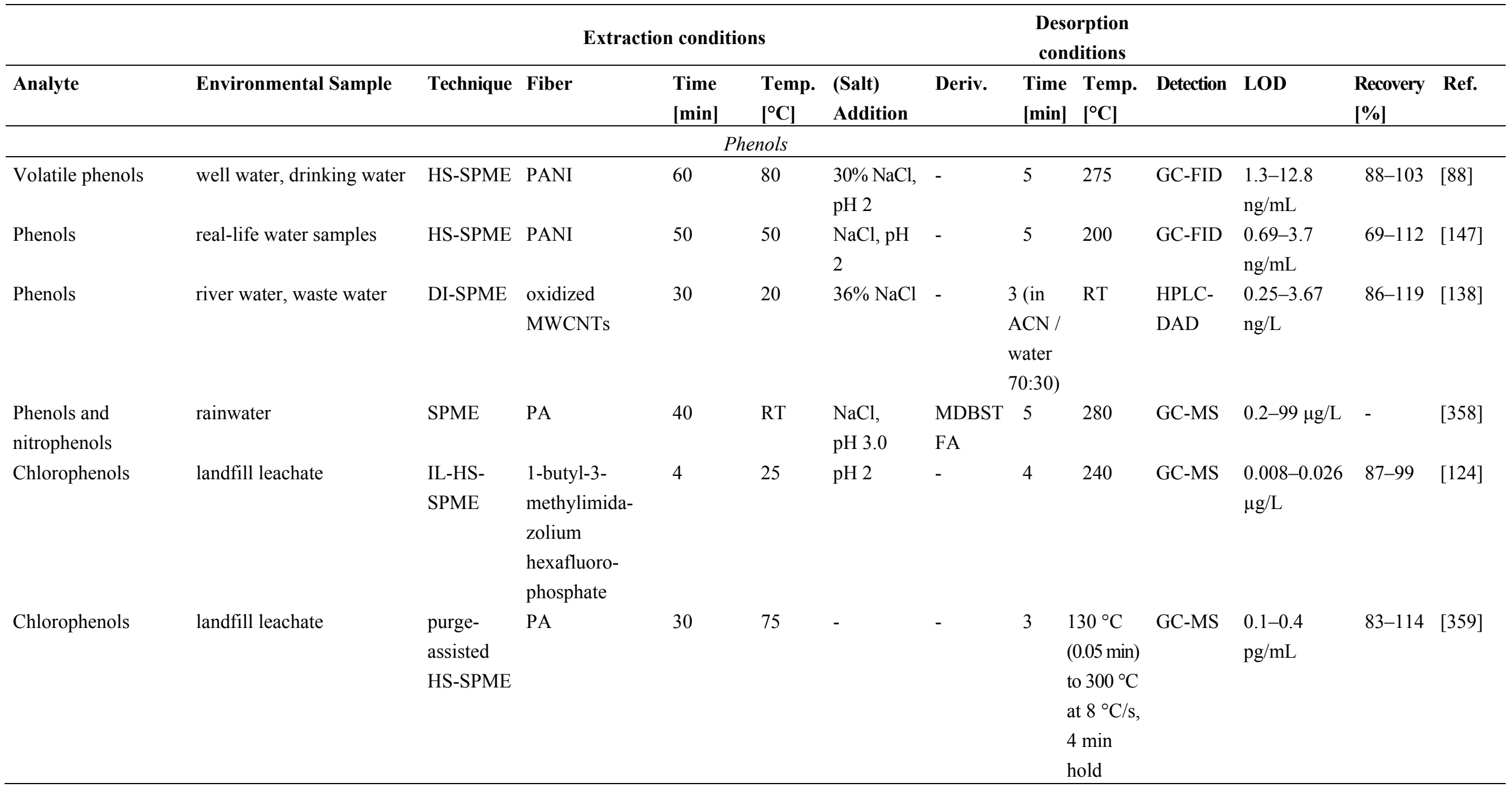


Table 4. Cont

\begin{tabular}{|c|c|c|c|c|c|c|c|c|c|c|c|c|c|}
\hline & & & Extraci & n condi & & & & $\begin{array}{l}\text { Desc } \\
\text { con }\end{array}$ & $\begin{array}{l}\text { orption } \\
\text { ditions }\end{array}$ & & & & \\
\hline Analyte & Environmental Sample & Technique & Fiber & $\begin{array}{l}\text { Time } \\
\text { [min] }\end{array}$ & $\begin{array}{l}\text { Temp. } \\
{\left[{ }^{\circ} \mathrm{C}\right]}\end{array}$ & $\begin{array}{l}\text { (Salt) } \\
\text { Addition }\end{array}$ & Deriv. & $\begin{array}{l}\text { Time } \\
\text { [min] }\end{array}$ & $\begin{array}{l}\text { Temp. } \\
{\left[{ }^{\circ} \mathrm{C}\right]}\end{array}$ & Detection & LOD & $\begin{array}{l}\text { Recovery } \\
{[\%]}\end{array}$ & Ref. \\
\hline \multicolumn{14}{|c|}{ Miscellaneous } \\
\hline $\begin{array}{l}\text { Organometallic } \\
\text { compounds } \\
\text { (mercury, lead, tin) }\end{array}$ & river water, seawater & HS-SPME & $\begin{array}{l}\text { PDMS-DVB- } \\
\text { CAR }\end{array}$ & 15 & 40 & - & $\begin{array}{l}\text { sodium } \\
\text { tetra- } \\
\text { ethyl- } \\
\text { borate }\end{array}$ & 2 & 260 & $\begin{array}{l}\text { GC-MS- } \\
\text { MS }\end{array}$ & $4-33 \mathrm{ng} / \mathrm{L}$ & $50-109$ & {$[360]$} \\
\hline $\begin{array}{l}\text { Organo-phosphorus } \\
\text { fire retardants and } \\
\text { plasticizers }\end{array}$ & $\begin{array}{l}\text { wastewater, MAE prior to } \\
\text { SPME }\end{array}$ & DI-SPME & PDMS & 30 & 65 & $10 \% \mathrm{NaCl}$ & - & 0.5 & 250 & $\begin{array}{l}\text { GC-ICP- } \\
\text { MS / } \\
\text { GC- } \\
\text { TOF-MS }\end{array}$ & $x^{2}$ & $38-43$ & {$[83]$} \\
\hline $\begin{array}{l}\text { Non-halogenated } \\
\text { solvents }\end{array}$ & textile wastewater & HS-SPME & PDMS & 10 & 35 & - & - & 5 & 240 & GC-MS & $\begin{array}{l}0.1-300 \\
\mu \mathrm{g} / \mathrm{L}\end{array}$ & - & {$[361]$} \\
\hline Acetone & seawater & SPME & PDMS-DVB & 30 & RT & $\begin{array}{l}\mathrm{NaCl}, \mathrm{pH} \\
3.7\end{array}$ & PFBHA & 5 & 250 & GC-MS & $3.0 \mathrm{nM}$ & - & {$[362]$} \\
\hline BTEX & waste water & HS-SPME & PDMS-DVB & 1 & RT & $35 \% \mathrm{NaCl}$ & - & 0.16 & 200 & $\begin{array}{l}\text { portable } \\
\text { GC- } \mu \text { FID }\end{array}$ & $0.4-1.4 \mu \mathrm{g} / \mathrm{L}$ & $98-111$ & [89] \\
\hline BTEX & groundwater & HS-SPME & PDMS-CAR & 15 & 25 & $\begin{array}{l}267 \mathrm{~g} / \mathrm{L} \\
\mathrm{NaCl}\end{array}$ & - & 2 & 290 & $\begin{array}{l}\text { cryo- } \\
\text { trap-GC- } \\
\text { MS }\end{array}$ & $\begin{array}{l}0.01-0.05 \\
\mathrm{ng} / \mathrm{L}\end{array}$ & - & {$[363]$} \\
\hline Acrolein & $\begin{array}{l}\text { surface water, drinking } \\
\text { water }\end{array}$ & HS-SPME & PDMS-CAR & 50 & 60 & $\begin{array}{l}\text { saturated } \\
\mathrm{NaCl} \\
\text { solution }\end{array}$ & $\begin{array}{l}2,2,2- \\
\text { trifluo- } \\
\text { roethyl- } \\
\text { hydra- } \\
\text { zine }\end{array}$ & - & 220 & GC-MS & $0.06 \mu \mathrm{g} / \mathrm{L}$ & $91-104$ & {$[364]$} \\
\hline
\end{tabular}


Table 4. Cont.

\begin{tabular}{|c|c|c|c|c|c|c|c|c|c|c|c|c|c|}
\hline & & & $\mathbf{E x}$ & ion conditic & & & & & $\begin{array}{l}\text { orption } \\
\text { ditions }\end{array}$ & & & & \\
\hline Analyte & Environmental Sample & Technique & Fiber & $\begin{array}{l}\text { Time } \\
\text { [min] }\end{array}$ & $\begin{array}{l}\text { Temp. } \\
{\left[{ }^{\circ} \mathrm{C}\right]}\end{array}$ & $\begin{array}{l}\text { (Salt) } \\
\text { Addition }\end{array}$ & Deriv. & $\begin{array}{l}\text { Time } \\
\text { [min] }\end{array}$ & $\begin{array}{l}\text { Temp. } \\
{\left[{ }^{\circ} \mathrm{C}\right]}\end{array}$ & Detection & LOD & $\begin{array}{l}\text { Recovery } \\
{[\%]}\end{array}$ & Ref. \\
\hline \multicolumn{14}{|c|}{ Miscellaneous } \\
\hline $\begin{array}{l}\text { Pharmaceutical } \\
\text { compounds }\end{array}$ & wastewater & dSPME & CW-TPR & 30 & 75 & $\begin{array}{l}300 \mathrm{~g} / \mathrm{L} \\
\mathrm{NaCl} \\
\mathrm{pH} \mathrm{3/11}\end{array}$ & - & 10 & - & $\begin{array}{l}\text { LC-MS- } \\
\text { MS }\end{array}$ & $\begin{array}{l}\text { LOQ: } 0.005- \\
0.05 \mu \mathrm{g} / \mathrm{L}\end{array}$ & $89-110$ & {$[90]$} \\
\hline $\begin{array}{l}\text { Endocrine disrupting } \\
\text { chemicals and } \\
\text { steroid hormones }\end{array}$ & river water & DI-SPME & $\mathrm{PA}$ & 90 & 45 & $\begin{array}{l}10 \mathrm{~g} / \mathrm{L} \\
\mathrm{NaCl} 10 \\
\mathrm{pH} 5\end{array}$ & BSTFA & 5 & 290 & GC-MS & $\begin{array}{l}0.002-0.378 \\
\mu \mathrm{g} / \mathrm{L}\end{array}$ & - & {$[365]$} \\
\hline Bisphenol A & landfill leachate & HS-SPME & $\mathrm{PA}$ & 60 & 25 & $\begin{array}{l}100 \mathrm{~g} / \mathrm{L} \\
\mathrm{NaCl}, \mathrm{pH} \\
2\end{array}$ & BSTFA & 5 & 280 & GC-MS & $0.03 \mu \mathrm{g} / \mathrm{L}$ & - & {$[91]$} \\
\hline Estrogens & surface water, wastewater & IT-SPME & DVB & $\begin{array}{l}20 \text { cycles } \\
\text { à } 40 \mu \mathrm{L} \\
(100 \mu \mathrm{L} / \mathrm{min})\end{array}$ & - & - & - & - & - & $\begin{array}{l}\text { LC-MS- } \\
\text { MS }\end{array}$ & $\begin{array}{l}2.7-11.7 \\
\mathrm{pg} / \mathrm{mL}\end{array}$ & 86-107 & {$[92]$} \\
\hline $\begin{array}{l}\text { Organic pollutants } \\
\text { (pesticides, } \\
\text { octyl/nonyl phenols, } \\
\text { pentachloro- } \\
\text { benzene, PAHs) }\end{array}$ & $\begin{array}{l}\text { wastewater, landfill } \\
\text { leachate }\end{array}$ & DI-SPME & CW-DVB & 45 & RT & $\mathrm{NaCl} 10 \%$ & - & 5 & 250 & $\begin{array}{l}\text { GC- } \\
\text { TOF-MS }\end{array}$ & - & - & {$[366]$} \\
\hline $\begin{array}{l}\text { Organochlorine } \\
\text { pesticides and } \\
\text { polychlorinated } \\
\text { bisphenyls }\end{array}$ & river water & HS-SPME & PDMS & 60 & 80 & - & - & 5 & 250 & $\begin{array}{l}\text { GC-MS- } \\
\text { MS }\end{array}$ & $0.4-26 \mathrm{pg} / \mathrm{L}$ & $75-105$ & [367] \\
\hline
\end{tabular}


Table 4. Cont.

\begin{tabular}{|c|c|c|c|c|c|c|c|c|c|c|c|c|c|}
\hline & & & Extracti & n conditior & & & & $\begin{array}{l}\text { Deso } \\
\text { cond }\end{array}$ & $\begin{array}{l}\text { rption } \\
\text { litions }\end{array}$ & & & & \\
\hline Analyte & Environmental Sample & Technique & Fiber & $\begin{array}{l}\text { Time } \\
{[\mathrm{min}]}\end{array}$ & $\begin{array}{l}\text { Temp. } \\
{\left[{ }^{\circ} \mathrm{C}\right]}\end{array}$ & $\begin{array}{l}\text { (Salt) } \\
\text { Addition }\end{array}$ & Deriv. & $\begin{array}{l}\text { Time } \\
\text { [min] }\end{array}$ & $\begin{array}{l}\text { Temp. } \\
{\left[{ }^{\circ} \mathrm{C}\right]}\end{array}$ & Detection & LOD & $\begin{array}{l}\text { Recovery } \\
{[\%]}\end{array}$ & Ref. \\
\hline \multicolumn{14}{|c|}{ Miscellaneous } \\
\hline $\begin{array}{l}\text { Volatile halogenated } \\
\text { hydrocarbons, } \\
\text { benzene }\end{array}$ & $\begin{array}{l}\text { groundwater from waste- } \\
\text { oil recycling facility }\end{array}$ & HS-SPDE & PDMS-AC & $\begin{array}{l}15 \text { cycles } \\
\text { à } 1 \mathrm{~mL} \\
(50 \\
\mu \mathrm{L} / \mathrm{s})\end{array}$ & 60 & $5 \% \mathrm{NaCl}$ & - & $\begin{array}{l}1 \mathrm{~mL} \\
(10 \\
\mu \mathrm{L} / \mathrm{s})\end{array}$ & 300 & GC-MS & $12-870 \mathrm{ng} / \mathrm{L}$ & - & [368] \\
\hline $\begin{array}{l}\text { BTEX and } \\
\text { halocarbons }\end{array}$ & contaminated water & HS-SPME & PAC & 20 & $40-45$ & $25 \% \mathrm{NaCl}$ & - & 1 & 260 & $\begin{array}{l}\text { GC-FID- } \\
\text { ECD }\end{array}$ & $\begin{array}{l}\text { LOQ: } 0.01- \\
0.94 \mu \mathrm{g} / \mathrm{L}\end{array}$ & $88-113$ & [369] \\
\hline Chlorobenzene & tap water, river water & HS-SPME & $\begin{array}{l}\text { nanofiber } \\
\text { coated by } \\
\text { electrospun PU }\end{array}$ & 5 & 30 & $25 \% \mathrm{NaCl}$ & - & 2 & 200 & GC-MS & $10 \mathrm{ng} / \mathrm{L}$ & $94-102$ & [370] \\
\hline $\begin{array}{l}\text { 1,2-cis- } \\
\text { dichloroethylene, } \\
\text { trichloroethylene }\end{array}$ & $\begin{array}{l}\text { effluent from soil column } \\
\text { experiments }\end{array}$ & HS-SPME & PDMS & 5 & 25 & - & - & 1 & 250 & GC-FID & $2.4-4.2 \mu \mathrm{g} / \mathrm{L}$ & - & [371] \\
\hline $\begin{array}{l}\text { 1,3-dichloro-2- } \\
\text { propanol }\end{array}$ & $\begin{array}{l}\text { tap water, river water, } \\
\text { paper mill sewage }\end{array}$ & HS-SPME & $\begin{array}{l}\text { PDMS-DVB- } \\
\text { CAR }\end{array}$ & 30 & 25 & $\mathrm{NaCl}$ & BSTFA & 5 & 260 & $\begin{array}{l}\text { GC-MS- } \\
\text { MS }\end{array}$ & $0.4 \mathrm{ng} / \mathrm{mL}$ & $93-103$ & [318] \\
\hline Epichlorohydrin & water and sewage & HS-SPME & PDMS-CAR & 15 & 50 & - & - & 1 & 240 & $\begin{array}{l}\text { GC-MS, } \\
\text { GC- } \\
\text { ECD }\end{array}$ & $\begin{array}{l}\text { water: } \\
1.0 \mathrm{ng} / \mathrm{L}\end{array}$ & - & [93] \\
\hline $\begin{array}{l}\text { Polybrominated } \\
\text { diphenyl ethers }\end{array}$ & river water, waste water & DI-SPME & MWCNT & 30 & RT & - & - & 2 & 295 & $\begin{array}{l}\text { GC- } \\
\text { ECD }\end{array}$ & $3.6-8.6 \mathrm{ng} / \mathrm{L}$ & $90-119$ & {$[372]$} \\
\hline
\end{tabular}


Table 4. Cont

\begin{tabular}{|c|c|c|c|c|c|c|c|c|c|c|c|c|c|}
\hline & & \multicolumn{6}{|c|}{ Extraction conditions } & \multicolumn{2}{|c|}{$\begin{array}{l}\text { Desorption } \\
\text { conditions }\end{array}$} & \multirow[b]{2}{*}{ Detection } & \multirow[b]{2}{*}{ LOD } & \multirow[b]{2}{*}{$\begin{array}{l}\text { Recovery } \\
{[\%]}\end{array}$} & \multirow[b]{2}{*}{ Ref. } \\
\hline Analyte & Environmental Sample & Technique & Fiber & $\begin{array}{l}\text { Time } \\
{[\mathrm{min}]}\end{array}$ & $\begin{array}{l}\text { Temp. } \\
{\left[{ }^{\circ} \mathrm{C}\right]}\end{array}$ & $\begin{array}{l}\text { (Salt) } \\
\text { Addition }\end{array}$ & Deriv. & $\begin{array}{l}\text { Time } \\
\text { [min] }\end{array}$ & $\begin{array}{l}\text { Temp. } \\
{\left[{ }^{\circ} \mathrm{C}\right]}\end{array}$ & & & & \\
\hline \multicolumn{14}{|c|}{ Soil / Sediment and other Solid Matrices } \\
\hline \multicolumn{14}{|c|}{ Odorous compounds } \\
\hline $\begin{array}{l}\text { VOC and odorous } \\
\text { compounds }\end{array}$ & dairy manure & HS-SPME & $\begin{array}{l}\text { PDMS-DVB- } \\
\text { CAR }\end{array}$ & 30 & 30 & - & - & - & 230 & $\begin{array}{l}\text { MD-GC- } \\
\text { MS-O }\end{array}$ & - & - & [373] \\
\hline $\begin{array}{l}\text { Odorous volatile } \\
\text { compounds }\end{array}$ & compost & HS-SPME & PDMS-CAR & 60 & 20 & - & - & 40 & 250 & GC-MS & $\begin{array}{l}0.06-2.38 \\
\mathrm{ppb}\end{array}$ & - & [374] \\
\hline \multicolumn{14}{|c|}{ Volatile and semivolatile organic compounds } \\
\hline VOC & $\begin{array}{l}\text { soil, manure, compost, } \\
\text { biochar }\end{array}$ & HS-SPME & $\begin{array}{l}\text { PDMS-DVB- } \\
\text { CAR }\end{array}$ & 20 & 20 & - & - & 5 & 230 & GC-MS & $\begin{array}{l}0.01-310 \\
\mathrm{ng} / \mathrm{g}\end{array}$ & - & [375] \\
\hline VOC & cow slurry & HS-SPME & $\begin{array}{l}\text { PDMS-DVB- } \\
\text { CAR / PDMS- } \\
\text { CAR }\end{array}$ & 15 & 35 & $\mathrm{NaCl}$ & - & 3 & 300 & GC-MS & $\begin{array}{l}0.02-1441 \\
\mu \mathrm{g} / \mathrm{L}\end{array}$ & - & [376] \\
\hline $\begin{array}{l}\text { Volatile and } \\
\text { semivolatile organic } \\
\text { compounds }\end{array}$ & urban landfill soil & HS-SPME & PDMS-DVB & 30 & 50 & $\mathrm{NaCl}$ & - & - & 250 & GC-MS & - & - & [377] \\
\hline \multicolumn{14}{|c|}{ Pesticides } \\
\hline $\begin{array}{l}\text { Pyrethroids, } \\
\text { organochlorine } \\
\text { pesticides }\end{array}$ & agricultural soils & HS-SPME & $\mathrm{PA}$ & 30 & 100 & - & - & 5 & 290 & $\begin{array}{l}\mathrm{GC}- \\
\mu \mathrm{ECD}\end{array}$ & $\begin{array}{l}0.004-1.2 \\
\mathrm{ng} / \mathrm{g}\end{array}$ & $71-147$ & [378] \\
\hline Pyrethroids & sediment pore water & SPME & PDMS & 20 & - & - & - & 3 & 260 & GC-ECD & - & - & [379] \\
\hline
\end{tabular}


Table 4. Cont

\begin{tabular}{|c|c|c|c|c|c|c|c|c|c|c|c|c|c|}
\hline & & \multicolumn{6}{|c|}{ Extraction conditions } & \multicolumn{2}{|c|}{$\begin{array}{l}\text { Desorption } \\
\text { conditions }\end{array}$} & \multirow[b]{2}{*}{ Detection } & \multirow[b]{2}{*}{ LOD } & \multirow[b]{2}{*}{$\begin{array}{l}\text { Recovery } \\
{[\%]}\end{array}$} & \multirow[b]{2}{*}{ Ref. } \\
\hline Analyte & Environmental Sample & Technique & Fiber & $\begin{array}{l}\text { Time } \\
\text { [min] }\end{array}$ & $\begin{array}{l}\text { Temp. } \\
{\left[{ }^{\circ} \mathrm{C}\right]}\end{array}$ & $\begin{array}{l}\text { (Salt) } \\
\text { Addition }\end{array}$ & Deriv. & $\begin{array}{l}\text { Time } \\
\text { [min] }\end{array}$ & $\begin{array}{l}\text { Temp. } \\
{\left[{ }^{\circ} \mathrm{C}\right]}\end{array}$ & & & & \\
\hline \multicolumn{14}{|c|}{$P A H$ and related compounds } \\
\hline PAH & $\begin{array}{l}\text { sediment pore water, } \\
\text { sediments }\end{array}$ & SPME & PDMS & $500 \mathrm{~h}$ & 18 & $\begin{array}{l}1 \mathrm{~mL} 10 \mathrm{mM} \\
\mathrm{NaN}_{3}\end{array}$ & - & 15 & & $\begin{array}{l}50{ }^{\circ} \mathrm{C} \text { to } \\
250{ }^{\circ} \mathrm{C} \text { at } \\
200^{\circ} \mathrm{C} \\
/ \mathrm{min}\end{array}$ & - & - & [380] \\
\hline PAH & $\begin{array}{l}\text { coastal sediments, MAE } \\
\text { with acetone prior to } \\
\text { SPME }\end{array}$ & DI-SPME & PDMS & 60 & 60 & - & - & - & 270 & GC-MS & $\begin{array}{l}0.07-0.76 \\
\mu \mathrm{g} / \mathrm{kg}\end{array}$ & $70-110$ & [381] \\
\hline PAH & sand, sediment & $\begin{array}{l}\text { CF-HS- } \\
\text { SPME }\end{array}$ & PDMS & 40 & $\begin{array}{l}150 \\
\text { (fiber: } \\
5 \text { ) }\end{array}$ & - & - & 2 & 300 & GC-FID & $0.3-3 \mathrm{pg} / \mathrm{g}$ & - & {$[54]$} \\
\hline $\begin{array}{l}\text { Parent and alkyl } \\
\text { PAH }\end{array}$ & sediment pore water & HS-SPME & PDMS & 30 & - & - & - & 5 & 320 & GC-MS & $\begin{array}{l}0.002-0.6 \\
\mathrm{ng} / \mathrm{mL}\end{array}$ & - & [382] \\
\hline \multicolumn{14}{|c|}{ Miscellaneous } \\
\hline $\begin{array}{l}\text { 4-t-octylphenol, } \\
\text { nonylphenol, } \\
\text { bisphenol A }\end{array}$ & activated sludge & nd-SPME & PA & - & 20 & - & - & 5 & 2800 & GC-MS & - & - & [383] \\
\hline Phenols and indoles & cow slurry & HS-SPME & $\begin{array}{l}\text { PDMS-DVB- } \\
\text { CAR }\end{array}$ & 15 & 35 & $\mathrm{NaCl}$ & $\begin{array}{l}\text { MTBST } \\
\text { FA }\end{array}$ & 9 & 300 & GC-MS & $\begin{array}{l}0.004-707 \\
\mu \mathrm{g} / \mathrm{L}\end{array}$ & $>64$ & {$[94]$} \\
\hline $\begin{array}{l}\text { Polybrominated } \\
\text { diphenyl ethers }\end{array}$ & soil & HS-SPME & $\begin{array}{l}\text { sol-gel M- } \beta- \\
\text { CD-OH-TSO }\end{array}$ & 60 & 95 & methanol & - & 12 & 300 & GC-MS & $\begin{array}{l}13.0-78.3 \\
\mathrm{pg} / \mathrm{g}\end{array}$ & 78-99 & [384] \\
\hline $\begin{array}{l}\text { Perfluoro-carboxylic } \\
\text { acids }\end{array}$ & $\begin{array}{l}\text { harbour sediments, PFE } \\
\text { prior to SPME }\end{array}$ & HS-SPME & PDMS & 30 & 30 & $\begin{array}{l}\text { saturated } \\
\mathrm{NaCl} \\
\text { solution }\end{array}$ & $\begin{array}{l}\text { boron } \\
\text { trifluo- } \\
\text { ride }\end{array}$ & 3 & 300 & GC-MS & $0.5-0.8 \mathrm{ng} / \mathrm{g}$ & $99-103$ & [95] \\
\hline
\end{tabular}


Table 4. Cont.

\begin{tabular}{|c|c|c|c|c|c|c|c|c|c|c|c|c|c|}
\hline & & & Extra & n condi & & & & $\begin{array}{l}\text { Deso } \\
\text { cond }\end{array}$ & $\begin{array}{l}\text { rption } \\
\text { litions }\end{array}$ & & & & \\
\hline Analyte & Environmental Sample & Technique & Fiber & $\begin{array}{l}\text { Time } \\
\text { [min] }\end{array}$ & $\begin{array}{l}\text { Temp. } \\
{\left[{ }^{\circ} \mathrm{C}\right]}\end{array}$ & $\begin{array}{l}\text { (Salt) } \\
\text { Addition }\end{array}$ & Deriv. & $\begin{array}{l}\text { Time } \\
{[\mathrm{min}]} \\
\end{array}$ & $\begin{array}{l}\text { Temp. } \\
{\left[{ }^{\circ} \mathrm{C}\right]}\end{array}$ & Detection & LOD & $\begin{array}{l}\text { Recovery } \\
{[\%]}\end{array}$ & Ref. \\
\hline \multicolumn{14}{|c|}{ Miscellaneous } \\
\hline $\begin{array}{l}\text { Organotin } \\
\text { compounds }\end{array}$ & sediment & HS-SPME & PDMS & 30 & 80 & $\begin{array}{l}\text { ethanol, } \\
\text { pH } 5.3\end{array}$ & $\begin{array}{l}\text { sodium } \\
\text { tetra- } \\
\text { ethyl- } \\
\text { borate }\end{array}$ & 1 & 250 & GC-MS & $\begin{array}{l}1.0-6.3 \\
\mu \mathrm{g} / \mathrm{kg}\end{array}$ & $98-117$ & {$[84]$} \\
\hline $\begin{array}{l}\text { Mono-, di- and tri- } \\
\text { butyltin }\end{array}$ & $\begin{array}{l}\text { sediment, extraction with } \\
\text { hydrochloric acid/ethanol } \\
\text { prior to SPME }\end{array}$ & HS-SPME & PDMS & 30 & 40 & $\mathrm{pH} 4$ & $\begin{array}{l}\text { sodium } \\
\text { tetra- } \\
\text { ethyl- } \\
\text { borate }\end{array}$ & 2 & 250 & $\begin{array}{l}\text { GC-MS- } \\
\text { MS }\end{array}$ & $\begin{array}{l}0.03-1.0 \\
\mathrm{pg} / \mathrm{g}\end{array}$ & - & {$[96]$} \\
\hline $\begin{array}{l}\text { Cyclopentadienyl- } \\
\text { manganese } \\
\text { tricarbonyl, } \\
\text { (methylcyclo-penta- } \\
\text { di-enyl)manganese } \\
\text { tricarbonyl }\end{array}$ & seawater, soil & HS-SPME & PDMS-DVB & 20 & 60 & $5 \% \mathrm{NaCl}$ & - & 0,25 & 250 & $\begin{array}{l}\text { GC- } \\
\text { MIP- } \\
\text { AED }\end{array}$ & $\begin{array}{l}0.62-0.65 \mathrm{pg} \\
\mathrm{Mn} / \mathrm{g}\end{array}$ & $76-113$ & {$[385]$} \\
\hline Nitrous oxide & $\begin{array}{l}\text { estuarine soils and } \\
\text { sediments }\end{array}$ & HS-SPME & PDMS-CAR & 2 & 50 & - & - & 1 & 200 & GC-MS & $18 \mathrm{ppb}$ & - & {$[386]$} \\
\hline
\end{tabular}

ACN: acetonitrile; CF: cold fiber; BSTFA: N,O-bis(trimethylsily)trifluoroacetamide; BTEX: benzene, toluene, ethyl benzene, ortho-xylene and meta- and para-xylene; BTX: benzene, toluene and xylene; DAD: diode array detector; deriv.: derivatization; DI: direct immersion; dSPME: dual SPME; ECD: electron capture detector; FID: flame ionization detector; FPD: flame photometric detector; IL: ionic liquid; IT: in-tube; FD: fluorescence detection; LOD: limit of detection; LOQ: limit of quantification; MA: microwave assisted; MAE: microwave assisted extraction; M- $\beta$-CD/OH-TSO: permethylated- $\beta$-cyclodextrin/hydroxyl-termination silicone oil; MD: multidimensional; MDBSTFA: N-(t-butyldimethylsilyl)-N-methyltrifluoroacetamide; MIP-AED: microwave-induced plasma atomic emission detection; MS-O: mass spectrometry-olfactometry; MTBSTFA: N-methyl-N-t-butyldimethylsilyltrifluoroacetamide; MW: multiwalled; nd-SPME: negligible depletion-SPME; NPD: nitrogen-phosphorus detector; PAC: powered activated carbon; PAHs: polycyclic aromatic hydrocarbons; PANI: polyaniline; PDMSAC: PDMS mixed with activated C; PFBHA: pentafluorobenzyl-hydroxylamine hydrochloride; PFE: pressurized fluid extraction; PIF: postcolumn photochemically induced fluorimetry derivatization; PMPVS: polymethylphenylvinylsiloxane; PU: polyurethane; ref.: reference; RT: room temperature; SW: single-walled; temp.: temperature; TOF: time-of-flight; TPR: template resin; TPS: total suspended particulate; TSD: thermoionic specific detection. 
Typical fields of applications are the analysis of VOC, odorous compounds, or micro-pollutants in indoor air, atmospheric air, or gaseous effluents from industrial or agricultural operations. For example, Bourdin and Desauziers [82] developed a simple and fast method based on on-fiber derivatization using BSTFA for the analysis of formaldehyde and other carbonyl compounds in indoor air. For a 5 min fiber exposure time, the limits of detection were below $0.5 \mu \mathrm{g} / \mathrm{m}^{3}$, the average reproducibility was $15 \%$, and the linearity of the calibration curves was satisfactory. Of the micro-pollutants, formaldehyde is of particular concern as it was recognized as "carcinogenic to humans" (class 1) by the International Agency for Research on Cancer. It is found in nearly every indoor atmosphere because of its numerous sources, such as furniture, human activity (tobacco), glues, and varnishes [82]. Several authors used SPME as a screening method for the characterization of odorous compounds in gaseous effluents, e.g., livestock operations [320] or production of poultry feather and viscera meal [325] in order to develop suitable abatement strategies. SPME can also be used for analysis of airborne particulate matter after collection on filters $[330,333]$.

\subsection{Aqueous Samples}

Aqueous sampling by SPME can be performed by direct immersion, the HS method, or the IT SPME method. Most of the papers reported HS-SPME applications to aqueous samples and subsequent GC separation combined with different detectors (see Table 4). In recent years DI-SPME followed by LC has gained popularity [21]. Derivatization techniques have been used for example for analysis of PAH and related compounds [357], phenols [337,358], organometallic compounds [360], endocrine disruptors and steroid hormones [91,365], acetone [362], and acrolein [364] in aqueous samples. In addition to commercially available SPME fibers, fibers with new coating materials have been used. They include poly(3,4-ethylene dioxythiophene) [146] and a sol-gel based amino fiber [351] for analysis of pesticides. Polyaniline [88,147], oxidized multiwalled carbon nanotubes [138], and 1-butyl-3-methylimidazolium hexafluorophosphate [124] have been applied to the analysis of phenols. Furthermore, a nanofiber coated by electrospun PU [370] was utilized for analysis of chlorobenzene.

SPME has been used for the analysis of a wide variety of water pollutants including pesticides, PAHs, and phenols. Several publications on SPME analysis of many classes of pesticides in groundwater, lake, river and seawater, and waste water are available [146,343-347]. Results showed good performance of the methods with high recovery rates $>70 \%$ and very low LOD, often in the range of $\mathrm{ng} / \mathrm{L}$. For example, Raposo et al. [347] applied a GC/ECD method using DI-SPME for the analysis of organochlorine pesticides in groundwater with recovery rates between 92 and 105\% and LOD between 0.0013 and $0.45 \mathrm{ng} / \mathrm{L}$ [347]. Furthermore, SPME has been used to determine PAH, a large group of molecules widely distributed in the environment, and their metabolites in tap, rain, and surface waters as well as scrubber water with high recoveries (>71\%) [353-357]. SPME has also been successfully applied to other classes of pollutants in environmental water samples, e.g., phenols [88,124,138,147,358,359], pharmaceuticals [90], endocrine disrupting chemicals and hormones [97,98,365] and organometallic compounds of mercury, lead and tin [360].

Other fields of SPME application are the analysis of volatile and semivolatile organic compounds in waste water and landfill leachate [340] and the analysis of odorous compounds like earthy-musty odorants [334-336] and volatile sulfur compounds [338] in tap, river, or lake water. 


\subsection{Solid Samples}

Sampling of soil and sediments as well as other solid materials like manure, activated sludge, and biochar by SPME is usually performed by HS methods. In some applications extraction of analytes has been assisted by heating of the sample [84,377,378,384-386] or cooling the fiber [54], use of microwaves [381]; use of pressurized fluid extraction [95], or addition of small amounts of organic solvents prior to SPME [84,384]. Most applications published on SPME analysis of solid environmental samples involve the use of commercially available SPME fibers. A novel sol-gel permethylated- $\beta$ cyclodextrin/hydroxyl-termination silicone oil coated fiber has been used to extract polybrominated diphenyl ethers from soil [384]. With this coating, improved fiber stability has been achieved at analytical characteristics similar to commercially available fibers (RSD $<10 \%$, LOD 13.0-78.3 pg/g, recovery $>78 \%, r>0.999$ ).

The main fields of SPME applications to solid environmental samples are analysis of pesticides [378,379], PAH and related compounds [380-382], phenols [94,383], perfluorocarboxylic acids [95], and organotin compounds [84] in contaminated soils and sediments (see Table 4). Since SPME is generally organic solvent free, rapid, and sensitive, it allows the detection of trace amounts of substances that are short-lived during biotransformation, thus, providing insight into degradation pathways. For example, Shen et al. [383] developed a negligible depletion-SPME (nd-SPME) method to measure free concentrations of 4-t-octylphenol, nonylphenol and bisphenol $\mathrm{A}$ in order to determine aerobic biodegradation kinetics in activated sludge [383]. In nd-SPME, the extracted amount of analytes is so small that the depletion (i.e., change of concentrations) of the analytes does not lead to a change in reaction kinetics or other relevant conditions in the sample. Further applications of SPME to solid environmental samples are characterization of VOC and odorous compounds in soil, manure, compost, and biochar samples [373-377].

\section{Prospects and Trends}

Recent trends in sample preparation techniques comprise miniaturization, automation, high-throughput performance, on-line coupling with analytical instruments as well as low-cost operation through extremely low or no solvent consumption. Among other techniques mentioned in this review, SPME has the potential to meet these challenges [50]. Through miniaturization of sample preparation steps time and operation costs can be saved. The main advantage however is the possibility to determine trace and ultratrace analytes in complex matrices.

In addition to SPME techniques, further selective extraction procedures have been developed. They all show clear trends toward simplification of sampling and sample preparation methods, an increase in reliability and precision, and the elimination of the cleanup step. The development of more sensitive and selective phases may be a step towards further miniaturization of these techniques. Increasing interest also lies in the field of automating sample preparation. Automation can result in faster procedures as well as improved precision and cost-effectiveness. Over the last decade, sample preparation devices have been automated and coupled to separation and detection systems specifically designed for automation.

Due to the lack of automation, SPME tends to be coupled more often to GC than to LC. Moreover, more recent techniques such as SBSE are not yet as widely accepted as SPME due to the difficulty of 
achieving full automation. Another challenge related to new SPME techniques that has to be overcome is the carry-over effect occurring for example in SPDE, in-tip SPME and MEPS techniques due to the remaining of analytes on the inner surface of the needle after heat desorption [50,63,64,387]. Another aspect that should be considered for optimization is the moderate enrichment capacity of these techniques [50].

Research regarding SPME is not only based on the development of new devices but also on the study of derivatization strategies, new coatings with higher extraction efficiencies, selectivity and stability, and the study of novel calibration processes [2]. During the SPME process there are many different parameters that can be applied to achieve specific methods. Derivatization for example is a valuable tool in many cases to achieve efficient extraction. With regard to chemical consumption and waste generation, a recent trend however is to avoid derivatization techniques where possible [98].

The variety of commercially available fiber coatings is constantly growing which open up the range for further applications. However, in addition to these commercially available sorbents, further promising coating procedures have been developed. This approach opens up new fields of application. Furthermore, efforts are made to overcome limitations in biocompatibility, on-site compatibility, selectivity and sensitivity of currently available SPME coatings [103,104]. Besides physical coating processes which are compatible with nearly all kinds of sorbent materials there is a clear trend towards procedures developed for specific components. Most attention in past years has been given to the application of nanomaterials [115,370]. Nanostructured MIP-based coatings will continue to be of particular interest. Great attention is currently given to such specific SPME coatings as a basis for development of more designable structures. Even when issues like selectivity for target analytes and applicability for samples with complex matrix compositions have to be solved [24].

Quantitation is currently the major challenge for SPME procedures to gain wider application. This review showed that calibration techniques are still under development [22]. The model calculation methods laid down in chapter 2.5 provide a meaningful approach to overcome these challenges. A better understanding of SPME processes will be the basis of further improvements. Nonetheless, a number of norms have already been implemented and indicate that the application of calibration methods in SPME is possible. In the field of aroma analysis of food, the main quantification barrier is the high complexity of volatile compounds [388,389].

Food safety and quality control is mainly responsible for the development of further rapid and automated procedures aimed to raise the sample throughput and minimize sources of error [3]. Since 2006 there has been a strong and still valid trend towards SPME application for the analysis of wine, fruit/vegetables, dairy and meat products and other foods. In this field, SPME seems to have a leading role followed by SBSE and liquid microextraction methods such as SDME and LLME [13].

In the field of environmental analysis, further improvement of rapid techniques suitable for on-site, on-line and in situ measurements of pollutants and their transformation products will be an important future challenge. These methods will provide insight into natural environmental processes, such as natural attenuation and uptake by plants as well as toxic effects and toxicity mechanisms of emerging chemicals.

Microextraction techniques represent a step towards the miniaturization of the analytical laboratory which is one of the major challenges and directions of future method development acitivites. Applications of SPME in different fields apart from environmental and food analysis such as pharmaceutical, process monitoring, clinical, forensic or drugs are continuously increasing [2]. 


\section{Conclusions}

Solid-phase microextraction (SPME) techniques take a leading position among microextraction methods due to their simplicity and the possibility of automation. SPME is a very useful technique for analyzing volatiles occurring in very low concentrations in various foods and environmental applications occurring in very low concentrations. A reliable tool for the identification and quantitation widely applied in the analysis of chemical compounds and biological substances needs a high-quality sample preparation. In this context, SPME may provide very good solutions. The development of new rapid SPME techniques might further increase its credibility. However, the challenges of this method in handling complex sample matrices must be taken into consideration. Additionally, limitations regarding quantitation of target analytes need to be reduced. The selection of an appropriate SPME fiber requires knowledge of the sample matrix properties. Nowadays, there is a large variety of different fiber coatings, extraction and desorption techqniues as well as derivatization procedures available, enabling the development of selective, sensitive and repeatable SPME methods for food and environmental analysis.

\section{Author Contributions}

Sybille Merkle reviewed the food applications and recent SPME developments together with Dr. Kim Karen Kleeberg who wrote to the environmental applications under the supervision of Prof. Jan Fritsche.

\section{Conflicts of Interest}

The authors declare no conflict of interest.

\section{References}

1. Nerín, C. Focus on sample handling. Anal. Bioanal. Chem. 2007, 388, 1001-1002.

2. Nerín, C.; Salafranca, J.; Aznar, M.; Batlle, R. Critical review on recent developments in solventless techniques for extraction of analytes. Anal. Bioanal. Chem. 2009, 393, 809-833.

3. Mondello, L.; Costa, R.; Tranchida, P.Q.; Dugo, P.; Presti, M.L.; Festa, S.; Fazio, A.; Dugo, G. Reliable characterization of coffee bean aroma profiles by automated headspace solid phase microextraction-gas chromatography-mass spectrometry with the support of a dual-filter mass spectra library. J. Sep. Sci. 2005, 28, 1101-1109.

4. Arthur, C.L.; Pawliszyn, J. Solid Phase Microextraction with Thermal Desorption Using Fused Silica Optical Fibers. Anal. Chem. 1990, 62, 2145-2148.

5. Pawliszyn, J. Solid Phase Microextraction: Theory and Practice; Wiley, VCH: New York, NY, USA, 1997.

6. Lord, H.; Pawliszyn, J. Evolution of solid-phase microextraction technology. J. Chromatogr. A 2000, 885, 153-193.

7. Adahchour, M.; Beens, J.; Vreuls, R.J.J.; Batenburg, A.M.; Rosing, E.A.E.; Brinkman, U.A.T. Application of Solid-Phase Micro-Extraction and Comprehensive Two-Dimensional Gas Chromatography (GC x GC) for Flavour Analysis. Chromatographia 2002, 55, 361-367. 
8. Chai, M.; Pawliszyn, J. Analysis of Environmental Air Samples by Solid-Phase Microextraction and Gas Chromatography/lon Trap Mass Spectrometry. Environ. Sci. Technol. 1995, 29, 693-701.

9. Kleeberg, K.K.; Dobberstein, D.; Hinrichsen, N.; Müller, A.; Weber, P.; Steinhart, H. Sampling Procedures with Special Focus on Automatization. In Advances in Food Diagnostics, 1st ed.; Nollet, L.M.L., Toldrá, F., Eds.; Blackwell Publishing: Ames, IA, USA, 2007; pp. 253-293.

10. Pawliszyn, J. Method and Device for Solid Phase Microextraction and Desorption. US Patent 5691206 A, 1997.

11. Dietz, C.; Sanz, J.; Cámara, C. Recent developments in solid-phase microextraction coatings and related techniques. J. Chromatogr. A 2006, 1103, 183-192.

12. Kataoka, H.; Lord, H.L.; Pawliszyn, J. Applications of solid-phase microextraction in food analysis. J. Chromatogr. A 2000, 880, 35-62.

13. Jeleń, H.H.; Majcher, M.; Dziadas, M. Microextraction techniques in the analysis of food flavor compounds: A review. Anal. Chim. Acta 2012, 738, 13-26.

14. Pillonel, L.; Bosset, J.O.; Tabacchi, R. Rapid Preconcentration and Enrichment Techniques for the Analysis of Food Volatile. A Review. Lebensm.-Wiss. Technol. 2002, 35, 1-14.

15. Wardencki, W.; Michulec, M.; Curylo, J. A review of theoretical and practical aspects of solid-phase microextraction in food analysis. Int. J. Food Sci. Technol. 2004, 39, 703-717.

16. Alpendurada, M.F. Solid-phase microextraction: A promising technique for sample preparation in environmental analysis. J. Chromatogr. A 2000, 889, 3-14.

17. Buchberger, W.; Zaborsky, P. Sorptive Extraction Techniques for Trace Analysis of Organic Pollutants in the Aquatic Environment. Acta Chim. Slov. 2007, 54, 1-13.

18. Ouyang, G.; Pawliszyn, J. Recent developments in SPME for on-site analysis and monitoring. TrAC Trends Anal. Chem. 2006, 25, 692-703.

19. Ribeiro, C.; Ribeiro, A.R.; Maia, A.S.; Gonçalves, V.M.; Tiritan, M.E. New Trends in Sample Preparation Techniques for Environmental Analysis. Crit. Rev. Anal. Chem. 2014, 44, 142-185.

20. Wardencki, W.; Curyło, J.; Namieśnik, J. Trends in solventless sample preparation techniques for environmental analysis. J. Biochem. Biophys. Methods 2007, 70, 275-288.

21. Padrón, M.E.; Afonso-Olivares, C.; Sosa-Ferrera, Z.; Santana-Rodríguez, J.J.; Microextraction Techniques Coupled to Liquid Chromatography with Mass Spectrometry for the Determination of Organic Micropollutants in Environmental Water Samples. Molecules 2014, 19, 10320-10349.

22. Ouyang, G.; Pawliszyn, J. A critical review in calibration methods for solid-phase microextraction. Anal. Chim. Acta 2008, 627, 184-197.

23. Aziz-Zanjani, M.O.; Mehdinia, A. Electrochemically prepared solid-phase microextraction coatings-A review. Anal. Chim. Acta 2013, 781, 1-13.

24. Aziz-Zanjani, M.O.; Mehdinia, A. A review on procedures for the preparation of coatings for solid phase microextraction. Microchim. Acta 2014, 181, 1169-1190.

25. Kumar, A.; Gaurav; Malik, A.K.; Tewary, D.K.; Singh, B. A review on development of solid phase Microextraction fibers by sol-gel methods and their applications. Anal. Chim. Acta 2008, 610, 1-14.

26. Luo, J.; Wu, Y.; Lin, S. Review: Recent developments of carbon nanotubes hybrid assemblies for sensing. Am. J. Nano Res. Appl. 2015, 3, 23-28.

27. Moein, M.M.; Said, R.; Bassyouni, F.; Abdel-Rehim, M. Solid Phase Microextraction and Related Techniques for Drugs in Biological Samples. J. Anal. Methods Chem. 2014, doi: 10.1155/2014/921350. 
28. Aulakh, J.S.; Malik, A.K.; Kaur, V.; Schmitt-Kopplin, P. A Review on Solid Phase Micro Extraction-High Performance Liquid Chromatography (SPME-HPLC) Analysis of Pesticides. Crit. Rev. Anal. Chem. 2005, 35, 71-85.

29. Turner, N.W.; Subrahmanyam, S.; Piletsky, S.A. Analytical methods for determination of mycotoxins: A review. Anal. Chim. Acta 2009, 632, 168-180.

30. Kataoka, H. Automated sample preparation using in-tube solid-phase microextraction and its application-A review. Anal. Bioanal. Chem. 2002, 373, 31-45.

31. Smith, R.M. Before the injection-Modern methods of sample preparation for separation techniques. J. Chromatogr. A 2003, 1000, 3-27.

32. Nerín, C.; Philo, M.R.; Salafranca, J.; Castle, L. Determination of bisphenol-type contaminants from food packaging materials in aqueous foods by solid-phase microextraction-highperformance liquid chromatography. J. Chromatogr. A 2002, 963, 375-380.

33. Zhang, Z.; Pawliszyn, J. Headspace solid-phase microextraction. Anal. Chem. 1993, 65, 1843-1852.

34. Tuduri, L.; Desauziers, V.; Fanlo, J.L. A simple calibration procedure for volatile organic compounds sampling in air with adsorptive solid-phase microextraction fibers. Analyst 2003, 128, $1028-1032$.

35. Spietelun, A.; Pilarczyk, M.; Kloskowski, A.; Namieśnik, J. Current trends in solid-phase microextraction (SPME) fibre coatings. Chem. Soc. Rev. 2010, 39, 4524.

36. Namieśnik, J.; Zygmunt, B.; Jastrzebska, A. Application of solid-phase microextraction for determination of organic vapours in gaseous matrices. J. Chromatogr. A 2000, 885, 405-418.

37. Souza-Silva, E.A.; Pawliszyn, J. Direct Immersion Solid-Phase Microextraction with MatrixCompatible Fiber Coating for Multiresidue Pesticide Analysis of Grapes by Gas ChromatographyTime-of-Flight Mass Spectrometry (DI-SPME-GC-ToFMS). J. Agric. Food Chem. 2015, 63, 4464-4477.

38. Aulakh, J.S.; Malik, A.K.; Kaur, V.; Schmitt-Kopplin, P. A Review on Solid Phase Micro Extraction-High Performance Liquid Chromatography (SPME-HPLC) Analysis of Pesticides. Crit. Rev. Anal. Chem. 2005, 35, 71-85.

39. Spietelun, A.; Marcinkowski, Ł.; Kloskowski, A.; Namieśnik, J. Application of green sample preparation techniques for the isolation, preconcentration and gas chromatographic determination of organic environmental pollutants. Proceeedings of the 6th Shanghai International Symposium on Analytical Chemistry, Shanghai, China, 16-18 October 2012.

40. Gan, J.; Bondarenko, S. Determination of Pesticides in Water. In Analysis of Pesticides in Food and Enviromental Samples; Tadeo, J.L., Ed.; CRC Press: Boca Raton, FL, USA, 2008; Volume 3, pp. 231-256.

41. Vas, G.; Vékey, K. Solid-phase microextraction: A powerful sample preparation tool prior to mass spectrometric analysis. J. Mass Spectrom. 2004, 39, 233-254.

42. Prosen, H.; Zupančič-Kralj, L. Solid-phase microextraction. TrAC Trends Anal. Chem. 1999, 18, 272-282.

43. Ai, J. Solid Phase Microextraction for Quantitative Analysis in Nonequilibrium Situations. Anal. Chem. 1997, 69, 1230-1236.

44. Ai, J. Headspace Solid Phase Microextraction. Dynamics and Quantitative Analysis before Reaching a Partition Equilibrium. Anal. Chem. 1997, 69, 3260-3266. 
45. Eisert, P.; Pawliszyn, J. Automated In-Tube Solid-Phase Microextraction Coupled to HighPerformance Liquid Chromatography. Anal. Chem. 1997, 69, 3140-3147.

46. Globig, D.; Weickhardt, C. Fully automated in-tube solid-phase microextraction for liquid samples coupled to gas chromatography. Anal. Bioanal. Chem. 2005, 381, 656-659.

47. Silva, C.; Cavaco, C.; Perestrelo, R.; Pereira, J.; Câmara, J.S. Microextraction by Packed Sorbent (MEPS) and Solid-Phase Microextraction (SPME) as Sample Preparation Procedures for the Metabolomic Profiling of Urine. Metabolites 2014, 4, 71-97.

48. Kubinec, R.; Berezkin, V.G.; Górová, R.; Addová, G.; Mracnová, H.; Soják, L. Needle concentrator for gas chromatographic determination of BTEX in aqueous samples. J. Chromatogr. B 2004, 800, 295-301.

49. Jochmann, M.A. Solventless extraction and enrichment methods for compound-specific isotope analysis. Ph.D. Thesis, University of Tubingen, Germany, 19 December 2006.

50. Kataoka, H. Recent developments and applications of microextraction techniques in drug analysis. Anal. Bioanal. Chem. 2010, 396, 339-364.

51. Saito, Y.; Jinno, K. Miniaturized sample preparation combined with liquid phase Separations. J. Chromatogr. A 2003, 1000, 53-67.

52. Zhang, Z.; Pawliszyn, J. Quantitative Extraction Using an Internally Cooled Solid Phase Microextraction Device. Anal. Chem. 1995, 67, 34-43.

53. Chen, Y.; Pawliszyn, J. Miniaturization and Automation of an Internally Cooled Coated Fiber Device. Anal. Chem. 2006, 78, 5222-5226.

54. Ghiasvand, A.R.; Hosseinzadeh, S.; Pawliszyn, J. New cold-fiber headspace solid-phase microextraction device for quantitative extraction of polycyclic aromatic hydrocarbons in sediment. J. Chromatogr. A 2006, 1124, 35-42.

55. Carasek, E.; Pawliszyn, J. Screening of Tropical Fruit Volatile Compounds Using Solid-Phase Microextraction (SPME) Fibers and Internally Cooled SPME Fiber. J. Agric. Food Chem. 2006, $54,8688-8696$.

56. Carasek, E.; Cudjoe, E.; Pawliszyn, J. Fast and sensitive method to determine chloroanisoles in cork using an internally cooled solid-phase microextraction fiber. J. Chromatogr. A 2007, 1138, $10-17$.

57. Mirnaghi, F.S.; Hein, D.; Pawliszyn, J. Thin-Film Microextraction Coupled with Mass Spectrometry and Liquid Chromatography-Mass Spectrometry. Chromatographia 2013, 76, 1215-1223.

58. Jiang, R.; Pawliszyn, J. Thin-film microextraction offers another geometry for solid-phase microextraction. TrAC Trends Anal. Chem. 2012, 39, 245-253.

59. Koziel, J.A.; Odziemkowski, M.; Pawliszyn, J. Sampling and Analysis of Airborne Particulate Matter and Aerosols Using In-Needle Trap and SPME Fiber Devices. Anal. Chem. 2001, 73, 47-54.

60. Wang, A.; Fang, F.; Pawliszyn, J. Sampling and determination of volatile organic compounds with needle trap devices. J. Chromatogr. A 2005, 1072, 127-135.

61. Saito, Y.; Ueta, I.; Kotera, K.; Ogawa, M.; Wada, H.; Jinno, K. In-needle extraction device designed for gas chromatographic analysis of volatile organic compounds. J. Chromatogr. A 2006, 1106, 190-195. 
62. SPDE ${ }^{\mathrm{TM}}$ : The Magic Needle. flyer; CHROMTECH GmbH: Idstein, Germany, 2006. Available online: http:/www.chromtech.de/download/20130130160105/CT-ProduktblattSPDE-231205.pdf (accessed on 31 March 2015).

63. Ampuero, S.; Bogdanov, S.; Bosset, J.O. Classification of unifloral honeys with an MS-based electronic nose using different sampling modes: SHS, SPME and INDEX. Eur. Food Res. Technol. 2004, 218, 198-207.

64. Lipinski, J. Automated solid phase dynamic extraction-Extraction of organics using a wall coated syringe needle. Fresenius. J. Anal. Chem. 2001, 369, 57-62.

65. Balasubramanian, S.; Panigrahi, S. Solid-Phase Microextraction (SPME) Techniques for Quality Characterization of Food Products: A Review. Food Bioprocess Technol. 2011, 4, 1-26.

66. Nilsson, T.; Ferrari, R.; Facchetti, S. Inter-laboratory studies for the validation of solid-phase microextraction for the quantitative analysis of volatile organic compounds in aqueous samples. Anal. Chim. Acta 1997, 356, 113-123.

67. Bene, A.; Luisier, J.L.; Fornage, A. Applicability of a SPME method for the rapid determination of VOCs. Chimia 2002, 56, 289-291.

68. Basheer, C.; Lee, H.K. Hollow fiber membrane-protected solid phase microextraction of triazine herbicides in bovine milk and sewage sludge samples. J. Chromatogr. A 2004, 1047, 189-194.

69. Razote, E.; Jeon, I.; Maghirang, R.; Chobpattana, W. Dynamic air sampling of volatile organic compounds using solid phase microextraction. J. Environ. Sci. Health Part B 2002, 37, 365-378.

70. Razote, E.B.; Maghirang, R.G.; Seitz, L.M.; Jeon, I.J. Characterization of volatile organic compounds on airborne dust in a swine finishing barn. Transac. ASAE 2004, 47, 1231-1238.

71. Parreira, F.V.; de Carvalho, C.R.; Cardeal, Z.L. Evaluation of indoor exposition to benzene, toluene, ethylbenzene, xylene and styrene by passive sampling with a solid-phase microextraction device. J. Chromatogr. Sci. 2002, 40, 122-126.

72. Jung, D.M.; Ebeler, S.E. Headspace solid-phase microextraction method for the study of the volatility of selected flavor compounds. J. Agric. Food Chem. 2003, 51, 200-205.

73. Pawliszyn, J. Solid phase Microextraction. In A century of Separation Science; Issaq, H.J., Ed.; Marcel Dekker, Inc: New York, NY, USA, 2002; pp. 399-419.

74. Costas-Rodriguez, M.; Pena-Pereira, F. Method Development with Miniaturized Sample Preparation Techniques. In Miniaturization in Sample Preparation; Pena-Pereira, F., Ed.; De Gruyter Open: Warsaw, Poland, 2014; pp. 276-307.

75. Pawliszyn, J. Handbook of solid phase Microextraction, 1st ed.; Elsevier Inc: Waltham, MA, USA, 2012.

76. Lord, H.L. Strategies for interfacing solid-phase microextraction with liquid chromatography. J. Chromatogr. A 2008, 1152, 2-13.

77. Tong, H.; Sze, N.; Thomson, B.; Nacson, S.; Pawliszyn, J. Solid phase microextraction with matrix assisted laser desorption/ionization introduction to mass spectrometry and ion mobility spectrometry. Analyst 2002, 127, 1207-1210.

78. Stalikas, C.D.; Fiamegos, Y.C. Microextraction combined with derivatization. TrAC Trends Anal. Chem. 2008, 27, 533-542.

79. Pan, L.; Pawliszyn, J. Derivatization/Solid-Phase Microextraction: New Approach to Polar Analytes. Anal. Chem. 1997, 69, 196-205. 
80. Martínez-Uruñuela, A.; González-Sáiz, J.M.; Pizarro, C. Multiple solid-phase microextraction in a non-equilibrium situation Application in quantitative analysis of chlorophenols and chloroanisoles related to cork taint in wine. J. Chromatogr. A 2005, 1089, 31-38.

81. Bianchi, F.; Careri, M.; Musci, M.; Mangia, A. Fish and food safety: Determination of formaldehyde in 12 fish species by SPME extraction and GC-MS analysis. Food Chem. 2007, 100, 1049-1053.

82. Bourdin, D.; Desauziers, V. Development of SPME on-fiber derivatization for the sampling of formaldehyde and other carbonyl compounds in indoor air. Anal. Bioanal. Chem. 2014, 406, 317-328.

83. Ellis, J.; Shah, M.; Kubachka, K.M.; Caruso, J.A. Determination of organophosphorus fire retardants and plasticizers in wastewater samples using MAE-SPME with GC-ICPMS and GC-TOFMS detection. J. Environ. Monit. 2007, 9, 1329-1336.

84. Devos, C.; Vliegen, M.; Willaert, B.; David, F.; Moens, L.; Sandra, P. Automated headspace-solidphase micro extraction-retention time locked-isotope dilution gas chromatography-mass spectrometry for the analysis of organotin compounds in water and sediment samples. J. Chromatogr. A 2005, 1079, 408-414.

85. Mateo-Vivaracho, L.; Ferreira, V.; Cacho, J. Automated analysis of 2-methyl-3-furanthiol and 3mercaptohexyl acetate at ng L-1 level by headspace solid-phase microextracion with on-fibre derivatisation and gas chromatography-negative chemical ionization mass spectrometric determination. J. Chromatogr. A 2006, 1121, 1-9.

86. Zhu, W.; Koziel, J.A.; Cai, L.; Özsoy, D.H.; Leeuwen, J.H. Quantification of Carbonyl Compounds Generated from Ozone-Based Food Colorants Decomposition Using On-Fiber Derivatization-SPME-GC-MS. Chromatography 2015, 2, 1-18.

87. Peter, A.; Köster, O.; Schildknecht, A.; Gunten, U. Occurrence of dissolved and particle-bound taste and odor compounds in Swiss lake waters. Water Res. 2009, 43, 2191-2200.

88. Mousavi, M.; Noroozian, E.; Jalali-Heravi, M.; Mollahosseini, A. Optimization of solid-phase microextraction of volatile phenols in water by a polyaniline-coated Pt-fiber using experimental design. Anal. Chim. Acta 2007, 581, 71-77.

89. Ji, J.; Deng, C.; Shen, W.; Zhang, X. Field analysis of benzene, toluene, ethylbenzene and xylene in water by portable gas chromatography-microflame ionization detector combined with headspace solid-phase microextraction. Talanta 2006, 69, 894-899.

90. Unceta, N.; Sampedro, M.C.; Bakar, N.K.A.; Gómez-Caballero, A.; Goicolea, M.A.; Barrio, R.J. Multi-residue analysis of pharmaceutical compounds in wastewaters by dual solid-phase microextraction coupled to liquid chromatography electrospray ionization ion trap mass spectrometry. J. Chromatogr. A 2010, 1217, 3392-3399.

91. Li, X.; Lin, L.; Zou, S.; Lan, C.; Luan, T. Determination of bisphenol A in landfill leachate by solid phase microextraction with headspace derivatization and gas chromatography-mass spectrophotometry. Chin. J. Anal. Chem. 2006, 34, 325-328.

92. Mitani, K.; Fujioka, M.; Kataoka, H. Fully automated analysis of estrogens in environmental waters by in-tube solid-phase microextraction coupled with liquid chromatography-tandem mass spectrometry. J. Chromatogr. A 2005, 1081, 218-224.

93. Gaca, J.; Wejnerowska, G. Determination of epichlorohydrin in water and sewage samples. Talanta 2006, 70, 1044-1050. 
94. Larreta, J.; Usobiaga, A.; Etxebarria, N.; Arana, G.; Zuloaga, O. Optimisation of the on-fibre derivatisation of volatile fatty acids in the simultaneous determination together with phenols and indoles in cow slurries. Anal. Bioanal. Chem. 2007, 389, 1603-1609.

95. Alzaga, R.; Salgado-Petinal, C.; Jover, E.; Bayona, J.M. Development of a procedure for the determination of perfluorocarboxylic acids in sediments by pressurised fluid extraction, headspace solid-phase microextraction followed by gas chromatographic-mass spectrometric determination. J. Chromatogr. A 2006, 1083, 1-6.

96. Carvalho, P.N.; Pinto, L.F.; Clara, M.; Basto, P.; Teresa, M.; Vasconcelos, S.D. Headspace solidphase micro-extraction and gas chromatography-ion trap tandem mass spectrometry method for butyltin analysis in sediments: Optimization and validation. Microchem. J. 2007, 87, 147-153.

97. Quintana, J.B.; Rodriguez, I. Strategies for the microextraction of polar organic contaminants in water samples. Anal. Bioanal. Chem. 2006, 384, 1447-1461.

98. Gałuszka, A.; Migaszewski, Z.; Namieśnik, J. The 12 principles of green analytical chemistry and the SIGNIFICANCE mnemonic of green analytical practices. TrAC Trends Anal. Chem. 2013, 50, 78-84.

99. Wu, J.; Xie, W.; Pawliszyn, J. Automated in tube solid-phase microextraction coupled with HPLCES-MS for the determination of catechins and caffeine in tea. Analyst 2000, 125, 2216-2222.

100. Górecki, T.; Yu, X.; Pawliszyn, J. Theory of analyte extraction by selected porous polymer SPME fibres. Analyst 1999, 124, 643-649.

101. Cai, J.; Liu, B.; Su, Q. Comparison of simultaneous distillation extraction and solid-phase microextraction for the determination of volatile flavor components. J. Chromatogr. A 2001, 930, $1-7$.

102. Kleeberg, K.K.; Liu, Y.; Jans, M.; Schlegelmilch, M.; Streese, J.; Stegrnann, R. Development of a simple and sensitive method for the characterization of odorous waste gas emissions by means of solid-phase microextraction (SPME) and GC MS/olfactometry. Waste Manage. 2005, 25, 872-879.

103. Huang, K.P.; Wang, G.R.; Huang, B.Y.; Liu, C.Y. Preparation and application of ionic liquidcoated fused-silica capillary fibers for solid-phase microextraction. Anal. Chim. Acta 2009, 645, $42-47$.

104. He, Y.; Pohl, J.; Engel R.; Rothman L.; Thomas, M. Preparation of ionic liquid based solid-phase microextraction fiber and its application to forensic determination of methamphetamine and amphetamine in human urine. J. Chromatogr. A 2009, 1216, 4824-4830.

105. Mullett, W.M.; Martin, P.; Pawliszyn, J. In-Tube Molecularly Imprinted Polymer Solid-Phase Microextraction for the Selective Determination of Propranolol. Anal. Chem. 2001, 73, 2383-2389.

106. Hofacker, S.; Mechtel, M.; Mager, M.; Kraus, H. Sol-gel: A new tool for coatings chemistry. Prog. Org. Coat. 2002, 45, 159-164.

107. Mullett, W.M.; Levsen, K.; Lubda, D.; Pawliszyn, J. Bio-compatible in-tube solid-phase microextraction capillary for the direct extraction and high-performance liquid chromatographic determination of drugs in human serum. J. Chromatogr. A 2002, 963, 325-334.

108. Chong, S.; Wang, D.; Hayes, J.; Wilhite, B.; Malik, A. Sol-gel coating technology for the preparation of solid-phase microextraction fibers of enhanced thermal stability. Anal. Chem. 1997, 69, 3889-3898.

109. Young, S.K. Sol-Gel Science for Ceramic Materials. Mater. Matt. 2006, 1, 8. 
110. Hu, B.; He, M.; Chen, B. Novel Materials in Solid-Phase Microextraction and Related Sample Preparation Approaches. In Miniaturization in Sample Preparation; Pereira, F.P., Ed.; De Gruyter Open Ltd: Warsaw, Poland; Berlin, Germany, 2014; pp. 88-190.

111. Djozan, D.; Assadi, Y.; Haddadi, S.H. Anodized Aluminum Wire as a Solid-Phase Microextraction Fiber. Anal. Chem. 2001, 73, 4054-4058.

112. Li, Q.; Ding, Y.; Yuan, D. Electrosorption-enhanced solid-phase microextraction of trace anions using a platinum plate coated with single-walled carbon nanotubes. Talanta 2011, 85, 1148-1153.

113. Li, M.K.; Lei, N.; Gong, C.; Yu, Y.; Lam, K.; Lam, M.H.; Yu, H.; Lam, P.K. An organically modified silicate molecularly imprinted solid-phase microextraction device for the determination of polybrominated diphenyl ethers. Anal. Chim. Acta 2009, 633, 197-203.

114. Ma, X.; Li, Q.; Yuan, D. Determination of endocrine-disrupting compounds in water by carbon nanotubes solid-phase microextraction fiber coupled online with high performance liquid chromatography. Talanta 2011, 85, 2212-2217.

115. Maghsoudi, S.; Noroozian, E. HP-SPME of Volatile Polycyclic Aromatic Hydrocarbons from Water Using Multiwalled Carbon Nanotubes Coated on a Steel Fiber through Electrophoretic Deposition. Chromatographia 2012, 75, 913-921.

116. Zewe, J.W.; Steach, J.K.; Olesik, S.V. Electrospun fibers for solid-phase microextraction. Anal. Chem. 2010, 82, 5341-5348.

117. Lin, B.; Li, T.; Zhao, Y.; Huang, F.-K.; Guo, L.; Feng, Y.-Q. Preparation of a TiO2 nanoparticledeposited capillary column by liquid phase deposition and its application in phosphopeptide analysis. J. Chromatogr. A 2008, 1192, 95-102.

118. Alizadeh, R.; Najafi, N.M.; Kharrazi, S. A new solid phase micro extraction for simultaneous head space extraction of ultra traces of polar and non-polar compounds. Anal. Chim. Acta 2011, 689, $117-121$.

119. Ji, J.; Liu, H.; Chen, J.; Zeng, J.; Huang, J.; Gao, L.; Wang, Y.; Chen, X. ZnO nanorod coating for solid phase microextraction and its applications for the analysis of aldehydes in instant noodle samples. J. Chromatogr. A 2012, 1246, 22-27.

120. Farhadi, K.; Matin, A.A.; Amanzadeh, H.; Biparva, P.; Tajik, H.; Farshid, A.A.; Pirkharrati, H. A novel dispersive micro solid phase extraction using zein nanoparticles as the sorbent combined with headspace solid phase micro-extraction to determine chlorophenols in water and honey samples by GC-ECD. Talanta 2014, 128, 493-499.

121. Anbia, M.; Khazaei, M. Ordered nanoporous carbon-based SPME and determination by GC. Chromatographia 2011, 73, 379-384.

122. Rahimi, A.; Hashemi, P.; Badiei, A.; Arab, P.; Ghiasvand, A.R. CMK-3 nanoporous carbon as a new fiber coating for solid-phase microextraction coupled to gas chromatography-mass spectrometry. Anal. Chim. Acta 2011, 695, 58-62.

123. Toledo, B.R.; Hantao, L.W.; Ho, T.D.; Augusto, F.; Anderson, J.L. A chemometric approach toward the detection and quantification of coffee adulteration by solid-phase microextraction using polymericionic liquid sorbent coatings. J. Chomatogr. A 2014, 1346, 1-7.

124. Ho, T.T.; Chen, C.Y.; Li, Z.G.; Yang, T.C.C.; Lee, M.R. Determination of chlorophenols in landfill leachate using headspace sampling with ionic liquid-coated solid-phase microextraction fibers combined with gas chromatography-mass spectrometry. Anal. Chim. Acta 2012, 712, 72-77. 
125. Yang, Y.; Chen, J.; Shi, J.P. Determination of diethylstilbestrol in milk using carbon nanotubereinforced hollow fiber solid-phase microextraction combined with high-performance liquid chromatography. Talanta 2012, 97, 222-228.

126. Sarafraz-Yazdi, A.; Abbasian, M.; Amiri, A. Determination of furan in food samples using two solid phase microextraction fibers based on sol-gel technique with gas chromatography-flame ionization detector. Food Chem. 2012, 131, 698-704.

127. Attari, S.G.; Bahrami, A.; Shahna, F.G.; Heidari, M. Solid-phase microextraction fiber development for sampling and analysis of volatile organohalogen compounds in air. J. Environ. Health Sci. Eng. 2014, 12, 123-130.

128. Shearrow, A.M.; Harris, G.A.; Fang, L.; Sekhar, P.K.; Nguyen, L.T.; Turner, E.B.; Bhansali, S.; Malik, A. Ionic liquid-mediated Sol-gel coatings for capillary microextraction. J. Chomatogr. A 2009, 1216, 5449-5458.

129. Shearrow, A.M.; Bhansali, S.; Malik, A. Ionic liquid-mediated bis(3-methyldimethoxysilyl)propyl. polypropylene oxide-based polar Sol-gel coatings for capillary microextraction. J. Chromatogr. A 2009, 1216, 6349-6355.

130. Zhou, X.; Shao, X.; Shu, J.J.; Liu, M.M.; Liu, H.L.; Feng, X.H.; Liu, F. Thermally stable ionic liquid-based Sol-gel coating for ultrasonic extraction-solid-phase microextraction-gas chromatography determination of phthalate esters in agricultural plastic films. Talanta 2012, 89, 129-135.

131. Liu, M.; Zhou, X.; Chen, Y.; Liu, H.; Feng, X.; Qiu, G.; Liu, F.; Zeng, Z. Innovative chemically bonded ionic liquids-based Sol-gel coatings as highly porous, stable and selective stationary phases for solid phase microextraction. Anal. Chim. Acta 2010, 683, 96-106.

132. Fan, Y.; Feng, Y.Q.; Shi, Z.G.; Wang, J.B. Ordered mesoporous silica coated capillary for in-tube solid phase microextraction coupled to high performance liquid chromatography. Anal. Chim. Acta 2005, 543, 1-8.

133. Zhu, F.; Liang, Y.; Xia, L.; Rong, M.; Su, C.; Lai, R.; Li, R.; Ouyang, G. Preparation and characterization of vinyl-functionalized mesoporous organosilica-coated solid-phase microextraction fiber. J. Chromatogr. A 2012, 1247, 42-48.

134. Moreira, M.A.; André, L.C.; Cardeal, Z.L. Analysis of plasticiser migration to meat roasted in plastic bags by SPME-GC/MS. Food Chem. 2015, 178, 195-200.

135. Es-Haghi, A.; Hosseini, S.M.; Khoshhesab, Z.M. Development and application of a new solidphase microextraction fiber by Solgel technology on titanium wire. Anal. Chim. Acta 2012, 742, 74-79.

136. Wang, Y.L.; Gao, Y.L.; Wang, P.P.; Shang, H.; Pan, S.Y.; Li, X.J. Sol-gel molecularly imprinted polymer for selective solid phase microextraction of organophosphorous pesticides. Talanta 2013, $115,920-927$.

137. Mu, L.; Hu, X.; Wen, J.; Zhou, Q. Robust aptamer Sol-gel solid phase microextraction of very polar adenosine from human plasma. J. Chromatogr. A 2013, 1279, 7-12.

138. Liu, X.; Ji, Y.; Zhang, Y.; Zhang, H.; Liu, M. Oxidized multiwalled carbon nanotubes as a novel solid-phase microextraction fiber for determination of phenols in aqueous samples. J. Chromatogr. A 2007, 1165, 10-17. 
139. Bagheri, H.; Ayazi, Z.; Sistani, H. Chemically bonded carbon nanotubes on modified gold substrate as novel unbreakable solid phase microextraction fiber. Microchim. Acta 2011, 174, 295-301.

140. Queiroz, M.E.C.; Oliveira, E.B.; Breton, F.; Pawliszyn, J. Immunoaffinity in-tube solid phase microextraction coupled with liquid chromatography-mass spectrometry for analysis of fluoxetine in serum samples. J. Chromatogr. A 2007, 1174, 72-77.

141. Hu, X.; Pan, J.; Hu, Y.; Huo, Y.; Li, G. Preparation and evaluation of solid-phase microextraction fiber based on molecularly imprinted polymers for trace analysis of tetracyclines in complicated samples. J. Chromatogr. A 2008, 1188, 97-107.

142. Djozan, D.; Farajzadeh, M.A.; Sorouraddin, S.M.; Baheri, T. Determination of methamphetamine, amphetamine and ecstasy by inside-needle adsorption trap based on molecularly imprinted polymer followed by GC-FID determination. Microchim. Acta 2012, 179, 209-217.

143. Amini, R.; Rouhollahi, A.; Adibi, M.; Mehdinia, A. A novel reusable ionic liquid chemically bonded fused-silica fiber for headspace solid-phase microextraction/gas chromatography-flame ionization detection of methyl tert-butyl ether in a gasoline sample. J. Chromatogr. A 2011, 1218, 130-136.

144. Pang, L.; Liu, J.F. Development of a solid-phase microextraction fiber by chemical binding of polymeric ionic liquid on a silica coated stainless steel wire. J. Chromatogr. A 2012, 1230, 8-14.

145. Tamer, U.; Ertas, N.; Udum, Y.A.; Sahin, Y.; Pekmez, K.; Yıldı, A. Electrochemically controlled solid-phase microextraction (EC-SPME) based on overoxidized sulfonated polypyrrole. Talanta 2005, 67, 245-251.

146. Banitaba, M.H.; Mohammadi, A.A.; Davarani, S.S.H.; Mehdinia, A. Preparation and evaluation of a novel solid-phase microextraction fiber based on poly(3,4-ethylenedioxythiophene) for the analysis of OCPs in water. Anal. Methods 2011, 3, 2061-2067.

147. Bagheri, H.; Babanezhad, A.M.E. An electropolymerized aniline-based fiber coating for solid phase microextraction of phenols from water. Anal. Chim. Acta 2005, 532, 89-95.

148. Chen, L.; Chen, W.; Ma, C.; Du, D.; Chen, X. Electropolymerized multiwalled carbon nanotubes/polypyrrole fiber for solid-phase microextraction and its applications in the determination of pyrethroids. Talanta 2011, 84, 104-108.

149. Mehdinia, A.; Khani, H.; Mozaffari, S. Fibers coated with a graphene-polyaniline nanocomposite for the headspace solid-phase microextraction of organochlorine pesticides from seawater samples. Microchim. Acta 2014, 181, 89-95.

150. Zhao, F.; Wang, M.; Ma, Y.; Zeng, B. Electrochemical preparation of polyaniline-ionic liquid based solid phase microextraction fiber and its application in the determination of benzene derivatives. J. Chromatogr. A 2011, 1218, 387-391.

151. Gao, Z.; Li, W.; Liu, B.; Liang, F.; He, H.; Yang, S.; Sun, C. Nanostructured polyaniline-ionic liquid composite film coated steel wire for headspace solid-phase microextraction of organochlorine pesticides in water. J. Chromatogr. A 2011, 1218, 6285-6291.

152. Mehdinia, A.; Mousavi, M.F.; Shamsipur, M. Nano-structured lead dioxide as a novel stationary phase for solid-phase microextraction. J. Chromatogr. A 2006, 1134, 24-31.

153. Budziak, D.; Martendal, E.; Carasek, E. Application of an NiTi alloy coated with ZrO2 solid-phase microextraction fiber for determination of haloanisoles in red wine samples. Microchim. Acta 2009, 164, 197-202. 
154. Liu, H.; Wang, D.; Ji, L.; Li, J.; Liu, S.; Liu, X.; Jiang, S. A novel TiO2 nanotube array/Ti wire incorporated solid-phase microextraction fiber with high strength, efficiency and selectivity. J. Chromatogr. A 2010, 1217, 1898-1903.

155. Cao, D.; Lü, J.; Liu, J.; Jiang, G. In situ fabrication of nanostructured titania coating on the surface of titaniumwire: A new approach for preparation of solid-phase microextraction fiber. Anal. Chim. Acta 2008, 611, 56-61.

156. Bagheri, H.; Aghakhani, A.; Baghernejad, M.; Akbarinejad, A. Novel polyamide-based nanofibers prepared by electrospinning technique for headspace solid-phase microextraction of phenol and chlorophenols from environmental samples. Anal. Chim. Acta 2012, 716, 34-39.

157. Newsome, T.E.; Zewe, J.W.; Olesik, S.V. Electrospun nanofibrous solid-phase microextraction coatings for preconcentration of pharmaceuticals prior to liquid chromatographic separations. $J$. Chromatogr. A 2012, 1262, 1-7.

158. Yu, Q.W.; Ma, Q.; Feng, Y. Temperature-response polymer coating for in-tube solid-phase microextraction coupled to highperformance. Talanta 2011, 84, 1019-1025.

159. International Organization for Standardization. Water Quality-Determination of Selected Plant Treatment Agents and Biocide Products-Method Using Solid-Phase Microextraction (SPME) Followed by Gas Chromatography-Mass Spectrometry (GC-MS); International Organization for Standardization: Genf, Switzerland, ISO 27108:2010.

160. Deutsches Institut für Normung. German Standard Methods for the Examination of Water, Waste Water and Sludge-Jointly Determinable Substances (group F)_Part 34: Determination of Selected Plant Treatment Agents, Biocides and Break-Down Products; Method Using Gas Chromatography (GC-MS) after Solid-Phase Micro Extraction (SPME) (F 34); Deutsches Institut für Normung: Berlin, Germany, DIN 38407-34:2006-05.

161. Deutsches Institut für Normung. German Standard Methods for the Examination of Water, Waste Water and Sludge-Jointly Determinable Substances (group F)-Part 41: Determination of Selected Easily Volatile Organic Compounds in Water-Method Using Gas Chromatography (GC-MS) after Headspace Solid-Phase Micro Extraction (HS-SPME) (F 41); Deutsches Institut für Normung: Berlin, Germany, DIN 38407-41:2011-06.

162. International Organization for Standardization. Water Quality-Determination of Volatile Organic Compounds in Water-Method Using Headspace Solid-Phase Micro-Extraction (HS-SPME) Followed by Gas Chromatography-Mass Spectrometry (GC-MS); International Organization for Standardization: Genf, Switzerland, ISO/DIS 17943:2014-07.

163. Austrian Standards Institute. Determination of Volatile Compounds in Cellulose-based Materials by Solid Phase Micro Extraction (SPME); Austrian Standards Insitute: Vienna, Austria, OENORM A 1117: 2004-05-01.

164. ASTM International. Standard Test Method for Determination of Parent and Alkyl Polycyclic Aromatics in Sediment Pore Water Using Solid-Phase Microextraction and Gas Chromatography/ Mass Spectrometry in Selected Ion Monitoring Mode; ASTM International: West Conshohocken, PA, USA, ASTM D 7363a:2013.

165. Bagheri, H.; Babanezhad, E.; Khalilian, F. A novel sol-gel-based amino-functionalized fiber for headspace solid-phase microextraction of phenol and chlorophenols from environmental samples. Anal. Chim. Acta 2008, 616, 49-55. 
166. Zeng, J.; Yu, B.; Chen, W.; Lin, Z.; Zhang, L.; Lin, Z.; Chen, X.; Wang, X. Application of ceramic/carbon composite as a novel coating for solid-phase Microextraction. J. Chromatogr. A 2008, 1188, 26-33.

167. Januszkiewicz, J.; Sabik, H.; Azarnia, S.; Lee, B. Optimization of headspace solid-phase microextraction for the analysis of specific flavors in enzyme modified and natural Cheddar cheese using factorial design and response surface methodology. J. Chromatogr. A 2008, 1195, 16-24.

168. Ravelo-Perez, L.M.; Hernandez-Borges, J.; Borges-Miquel, T.M.; Rodriguez Delgado, M.A. Multiple pesticide analysis in wine by MEKC combined with solid-phase microextraction and sample stacking. Electrophoresis 2007, 28, 4072-4081.

169. De Jager, L.S.; Perfetti, G.A.; Diachenko, G.W. Analysis of tetramethylene disulfotetramine in foods using solid-phase microextraction-gas chromatography-mass spectrometry. J. Chromatogr. A 2008, 1192, 36-40.

170. Saison, D.; De Schutter, D.P.; Delvaux, F.; Delvaux, F.R. Optimisation of a complete method for the analysis of volatiles involved in the flavour stability of beer by solid-phase microextraction in combination with gas chromatography and mass spectrometry. J. Chromatogr. A 2008, 1190, 342-349.

171. Iglesias, J.; Medina, I. Solid-phase microextraction method for the determination of volatile compounds associated to oxidation of fish muscle. J. Chromatogr. A 2008, 1192, 9-16.

172. Plutowska, B.; Wardencki, W. Determination of volatile fatty acid ethyl esters in raw spirits using solid phase microextraction and gas chromatography. Anal. Chim. Acta 2008, 613, 64-73.

173. Peldszus, S. Organic Acids. In Chromatographic Analysis of the Environment, 3rd ed.; Nollet, L.M.L, Ed.; CRC Press: Boca Raton, FL, USA, 2006; Volume 93, pp. 453-512.

174. Chee, K.K.; Wong, M.K.; Lee, H.K. SPME for the Determination of Organochlorine Pesticides. In Applications of Solid Phase Microextraction; Pawliszyn, J., Ed.; The Royal Society of Chemistry: Cambridge, UK, 1999; pp. 212-226.

175. Bocchini, P.; Pinelli, F.; Pozzi, R.; Ghetti, F.; Galletti, G.C. Quantitative determination of dimethyl fumarate in silica gel by solid-phase microextraction/gas chromatography/mass spectrometry and ultrasound-assisted extraction/gas chromatography/mass spectrometry. Environ. Monit. Assess. 2015, 187, 320.

176. Ezquerro, S.; Pons, B.; Tena, M.T. Direct quantitation of volatile organic compounds in packaging materials by headspace solid-phase microextraction-gas chromatography-mass spectrometry. J. Chromatogr. A 2003, 985, 247-257.

177. Wu, C.-M.; Wang, Z. Volatile Compounds in Fresh and Processed Shiitake Mushrooms (Lentinus edodes Sing.). Food Sci. Technol. Res. 2000, 6, 166-170.

178. Djozan, D.; Jouyban, A.; Norouzi, J. Ultrasonic assisted SPME coupled with GC and GC-MS using pencil lead as a fiber for monitoring the organic volatile impurities of ceftazidime. J. Chromatogr. Sci. 2008, 46, 680-685.

179. McGowin, A.E. Polycyclic Aromatic Hydrocarbons. In Chromatographic Analysis of the Environment, 3rd ed.; Nollet, L.M.L, Ed.; CRC Press: Boca Raton, FL, USA, 2006; Volume 93, pp. 555-616. 
180. Luukkonen, V. Determination of chlorophenols from water by solid phase microextraction-Ion mobility spectrometry (SPME-IMS). Master's thesis, Lappeenranta University of Technology, Lappeenranta, Finland, 29 November 2012.

181. Larroque, V.; Desauziers, V.; Mocho, P. Development of a solid phase microextraction (SPME) method for the sampling of VOC traces in indoor air. J. Environ. Monit. 2006, 8, 106-111.

182. Qin, Z.; Bragg, L.; Ouyang, G.; Pawliszyn, J. Comparison of thin-film microextraction and stir bar sorptive extraction for the analysis of polycyclic aromatic hydrocarbons in aqueous samples with controlled agitation conditions. J. Chromatogr. A 2008, 89, 1196-1197.

183. Flores, M.; Hernández, D. Optimization of Multiple Headspace Solid-Phase Microextraction for the Quantification of Volatile Compounds in Dry Fermented Sausages. J. Agric. Food Chem. 2007, $55,8688-8695$.

184. Paschke, A.; Vrana, B.; Popp, P.; Schürmann, G. Comparative application of solid-phase microextraction fibre assemblies and semi-permeable membrane devices as passive air samplers for semi-volatile chlorinated organic compounds. A case study on the landfill "Grube Antonie" in Bitterfeld, Germany. Environ. Pollut. 2006, 144, 414-422.

185. Ouyang, G.; Zhao, W.; Alaee, M.; Pawliszyn, J. Time-weighted average water sampling with a diffusion-based solid-phase microextraction device. J. Chromatogr. A 2007, 1138, 42-46.

186. Koziel, J.; Jia, M.; Pawliszyn, J. Air Sampling with Porous Solid-Phase Microextraction Fibers. Anal. Chem. 2000, 72, 5178-5186.

187. Chen, Y.; Koziel, Y.A.; Pawliszyn, J. Calibration for On-Site Analysis of Hydrocarbons in Aqueous and Gaseous Samples Using Solid-Phase Microextraction. Anal. Chem. 2003, 75, 6485-6493.

188. Zhao, W.; Ouyang, G.; Alaee, M.; Pawliszyn, J. On-rod standardization technique for timeweighted average water sampling with a polydimethylsiloxane rod. J. Chromatogr. A 2006, 1124, $112-120$.

189. Bragg, L.; Qin, Z.; Alaee, M.; Pawliszyn, J. Field Sampling with a Polydimethylsiloxane Thin-Film. J. Chromatogr. Sci. 2006, 44, 317-323.

190. Ouyang, G.; Cai, J.; Zhang, X.; Li, H.; Pawliszyn, J. Standard-free kinetic calibration for rapid on-site analysis by solid-phase microextraction. J. Sep. Sci. 2008, 31, 1167-1172.

191. Khaled, A.; Pawliszyn, J. Time-weighted average sampling of volatile and semi-volatile airborne organic compounds by the solid-phase Microextraction device. J. Chromatogr. A 2000, 892, 455-467.

192. Chen, Y.; O’Reilly, J.; Wang, Y.; Pawliszyn, J. Standards in the extraction phase, a new approach to calibration of Microextraction processes. Analyst 2004, 129, 702-703.

193. Zhao, W.; Ouyang, G.; Pawliszyn, J. and application of in-fibre internal standardization solid-phase microextraction. Analyst 2007, 132, 256-261.

194. Weurman, S.; van Lunteren, G. Application of gas chromatography to the study of food odours. In Aroma- and Flavour-Producing Substances in Foods; Solms, J., Neukom, S., Eds.; Forster: Zurich, Switzerland, 1967; pp. 21-34.

195. Rijkens, F.; Boelens, M.H. The future of aroma research. In Aroma Research; Proceedings of the International Symposium on Aroma Research, Central Institute for Nutrition and Food Research TNO, Zeist, Netherlands, 26-29 May 1975; Maarse, H., Groenen, P.J., Eds.; Pudoc: Wageningen, The Netherlands, 1975; pp. 203-222. 
196. Pawliszyn, J. Sample Preparation: Quo Vadis? Anal. Chem. 2003, 75, 2543-2558.

197. Pawliszyn, J. Handbook of Solid Phase MICROEXTRACTION; University of Waterloo: Waterloo, Canada, 2007.

198. Ferreira, S.L.C.; Dos Santos, W.N.L.; Quintella, C.M.; Neto, B.B.; Bosque-Sendra, J.M. Doehlert matrix: A chemometric tool for analytical chemistry-Review. Talanta 2004, 63, 1061-1067.

199. Risticevic, S.; Carasek, E.; Pawliszyn, J. Headspace solid-phase microextraction-gas chromatographic-time-of-flight mass spectrometric methodology for geographical origin verification of coffee. Anal. Chim. Acta 2008, 617, 72-84.

200. Chen, Y.; Shirey, R.E.; Sidisky, L.M. Determination of Diacetyl in Butter and Air Samples by SPME Coupled with GC-MS. Chromatographia 2010, 72, 999-1004.

201. Mallia, S.; Fernández-Garcia, E.; Bosset, J.O. Comparison of purge and trap and solid phase microextraction techniques for studying the volatile aroma compounds of three European PDO hard cheeses. Int. Dairy J. 2005, 15, 741-758.

202. Majcher, M.A.; Kaczmarek, A.; Klensporf-Pawlik, D.; Pikul, J.; Jeleń, H.H. SPME-MS-Based Electronic Nose as a Tool for Determination of Authenticity of PDO Cheese, Oscypek. Food Anal. Methods 2015, doi: 10.1007/s12161-015-0114-X.

203. Ocak, E.; Javidipour, I.; Tuncturk, Y. Volatile compounds of Van Herby cheeses produced with raw and pasteurized milks from different species. J. Food Sci. Technol. 2014, doi: 10.1007/s13197014-1458-8.

204. Tunick, M.H.; Iandola, S.K.; Hekken, D.L. Comparison of SPME Methods for Determining Volatile Compounds in Milk, Cheese, and Whey Powder. Foods 2013, 2, 534-543.

205. Xie, J.; Sun, B.; Zheng, F.; Wang, S. Volatile flavor constituents in roasted pork of Mini-pig. Food Chem. 2008, 109, 506-514.

206. Yu, A.N.; Sun, B.G.; Tian, D.T.; Qu, W.Y. Analysis of volatile compounds in traditional smokecured bacon (CSCB) with different fiber coatings using SPME. Food Chem. 2008, 110, 233-238.

207. Argyri, A.A.; Mallouchos, A.; Panagou, E.Z.; Nychas, G.J.E. The dynamics of the HS/SPME$\mathrm{GC} / \mathrm{MS}$ as a tool to assess the spoilage of minced beef stored under different packaging and temperature conditions. Int. J. Food Microbiol. 2015, 193, 51-58.

208. Benet, I.; Guàrdia, M.D.; Ibañez, C.; Solà, J.; Arnau, J.; Roura, E. Analysis of SPME or SBSE extracted volatile compounds from cooked cured pork ham differing in intramuscular fat profiles. Food Sci. Technol. 2015, 60, 393-399.

209. Corral, S.; Salvador, A.; Flores, M. Salt reduction in slow fermented sausages affects the generation of aroma active compounds. Meat Sci. 2013, 93, 776-785.

210. Gardini, F.; Tabanelli, G.; Lanciotti, R.; Montanari, C.; Luppi, M.; Coloretti, F.; Chiavari, C.; Grazia, L. Biogenic amine content and aromatic profile of Salama da sugo, a typical cooked fermented sausage produced in Emilia Romagna Region (Italy). Food Control 2013, 32, 638-643.

211. Ma, Q.L.; Hamid, N.; Bekhit, A.E.D.; Robertson, J.; Law, T.F. Optimization of headspace solid phase microextraction (HS-SPME) for gas chromatography mass spectrometry (GC-MS) analysis of aroma compounds in cooked beef using response surface methodology. Microchem. J. 2013, $111,16-24$. 
212. Câmara, J.S.; Marques, J.C.; Perestrelo, R.M.; Rodrigues, F.; Oliveira, L.; Andrade, P.; Caldeira, M. Comparative study of the whisky aroma profile based on headspace solid phase microextraction using different fibre coatings. J. Chromatogr. A 2007, 1150, 198-207.

213. Capobiango, M.; Mastello, R.B.; Chin, S.T.; Oliveira, E.S.; Cardeal, Z.L.; Marriott, P.J. Identification of aroma-active volatiles in banana Terra spirit usingmultidimensional gas chromatography with simultaneous massspectrometry and olfactometry detection. J. Chromatogr. A 2015, 1388, 227-235.

214. Du, L.; He, T.; Li, W.; Wang, R.; Xiao, D. Analysis of volatile compounds in Chinese Laobaigan liquor using headspace solid-phase microextraction coupled with GC-MS. Anal. Methods 2015, 7, 1906-1913.

215. Giraudel, J.L.; Setkova, L.; Pawliszyn, J.; Montury, M. Rapid headspace solid-phase microextraction-gas chromatographic-time-of-flight mass spectrometric method for qualitative profiling of ice wine volatile fraction III. Relative characterization of Canadian and Czech ice wines using self-organizing maps. J. Chromatogr. A 2007, 1147, 241-253.

216. Setkova, L.; Risticevic, S.; Pawliszyn, J. Rapid headspace solid-phase microextraction-gas chromatographic-time-of-flight mass spectrometric method for qualitative profiling of ice wine volatile fraction II: Classification of Canadian and Czech ice wines using statistical evaluation of the data. J. Chromatogr. A 2007, 1147, 224-240.

217. Jurado, J.M.; Ballesteros, O.; Alcázar, A.; Pablos, F.; Martín, M.J.; Vilchez, J.L.; Navalón, A. Differentiation of certified brands of origins of Spanish white wines by HS-SPME-GC and chemometrics. Anal. Bioanal. Chem. 2008, 390, 961-970.

218. Xiao, Z.; Zhou, X.; Niu, Y.; Yu, D.; Zhu, J.; Zhu, G. Optimization and application of headspacesolid-phasemicro-extraction coupled with gas chromatography-massspectrometry for the determination of volatile compounds in cherrywines. J. Chromatogr. B 2015, 978-979, 122-130.

219. Wang, X.; Xie, K.; Zhuang, H.; Ye, R.; Fang, Z.; Feng, T. Volatile flavor compounds, total polyphenolic contents and antioxidant activities of a China gingko wine. Food Chem. 2015, 182, 41-46.

220. Kim, B.H.; Park, S.K. Volatile aroma and sensory analysis of black raspberry wines fermented by different yeast strains. J. Inst. Brew. 2015, 121, 87-94.

221. Liu, M.; Zeng, Z.; Xiong, B. Preparation of novel solid-phase microextraction fibers by sol-gel technology for headspace solid-phase microextraction-gas chromatographic analysis of aroma compounds in beer. J. Chromatogr. A 2005, 1065, 287-299.

222. Braga, C.G.; Prado, A.; Pinto, J.S.; Alencar, S.M. Volatile profile of yellow passion fruit juice by static headspace and solid phase microextraction techniques. Cienc. Rural 2015, 45, 356-363.

223. Caven-Quantrill, D.J.; Buglass, A.J. Comparison of micro-scale simultaneous distillationextraction and stir bar sorptive extraction for the determination of volatile organic constituents of grape juice. J. Chromatogr. A 2006, 1117, 121-131.

224. Mirhosseini, H.; Salmah, Y.; Nazimah, S.A.H.; Tan, C.P. Solid-phase microextraction for headspace analysis of key volatile compounds in orange beverage emulsion. Food Chem. 2007, $105,1659-1670$. 
225. Várvölgyi, E.; Gere, A.; Szöllösi, D.; Sipos, L.; Kovács, Z.; Kókai, Z.; Csóka, M.; Mednyánszky, Z.; Fekete, A.; Korány, K. Application of Sensory Assessment, Electronic Tongue and GC-MS to Characterize Coffee Samples. Arab. J. Sci. Eng. 2015, 40, 125-133.

226. Barié, N.; Bücking, M.; Stahl, U.; Rapp, M. Detection of coffee flavour ageing by solid-phase microextraction/surface acoustic wave sensor array technique (SPME/SAW). Food Chem. 2015, $176,212-218$.

227. Evangelista, S.R.; Pedrozo, M.G.; Miguel, C.P.; Cordeiro, C.S.; Silva, C.F.; Pinheiro, A.C.M.; Schwan, R.F. Inoculation of starter cultures in a semi-dry coffee (Coffea arabica) fermentation process. Food Microbiol. 2014, 44, 87-95.

228. Guillot, S.; Peytavi, L.; Bureau, S.; Boulanger, R.; Lepoutre, J.P.; Crouzet, J.; Schorr-Galindo, S. Aroma characterization of various apricot varieties using headspacesolid phase microextraction combined with gas chromatography-mass spectrometry and gas chromatography-olfactometry. Food Chem. 2006, 96, 147-155.

229. Solis-Solis, H.M.; Calderón-Santoyo, M.; Schorr-Galindo, S.; Luna-Solano, G.; Ragazzo-Sánchez, J.A. Characterization of aroma potential of apricot varieties using different extraction techniques. Food Chem. 2007, 105, 829-837.

230. Gokbulut, I.; Karabulut, I. SPME-GC-MS detection of volatile compounds in apricot varieties. Food Chem. 2012, 132, 1098-1102.

231. Ong, B.T.; Nazimah, S.A.H.; Tan, C.P.; Mirhosseini, H.; Osman, A.; Hashim, D.M.; Rusul, G. Analysis of volatile compounds in five jackfruit (Artocarpus heterophyllus L.) cultivars using solid-phase microextraction (SPME) and gas chromatography-time-of-flight mass spectrometry (GC-TOFMS). J. Food Compos. Anal. 2008, 21, 416-422.

232. Kralj, M.B.; Jug, T.; Komel, E.; Fajt, N.; Jarni, K.; Živković, J.; Mujić, I. Aromatic compound in different peach cultivars and effect of preservatives on the final aroma of cooked fruits. Hem. Ind. 2014, 68, 767-779.

233. Spínola, V.; Perestrelo, R.; Câmara, J.S.; Castilho, P.C. Establishment of Monstera deliciosa fruit volatile metabolomic profile at different ripening stages using solid-phase microextraction combined with gas chromatography-mass spectrometry. Food Res. Int. 2015, 67, 409-417.

234. Steingass, C.B.; Carle, R.; Schmarr, H.G. Ripening-dependent metabolic changes in the volatiles of pineapple (Ananas comosus (L.) Merr.) fruit: I. Characterization of pineapple aroma compounds by comprehensive two-dimensional gas chromatography-mass spectrometry. Anal. Bioanal. Chem. 2015, 407, 2591-2608.

235. Vavoura, M.V.; Badeka, A.V.; Kontakos, S.; Kontominas, M.G. Characterization of Four Popular Sweet Cherry Cultivars Grown in Greece by Volatile Compound and Physicochemical Data Analysis and Sensory Evaluation. Molecules 2015, 20, 1922-1940.

236. Wang, D.; Cai, J.; Zhu, B.Q.; Wu, G.F.; Duan, C.Q.; Chen, G.; Shi, Y. Study of free and glycosidically bound volatile compounds in air-dried raisins from three seedless grape varieties using HS-SPME with GC-MS. Food Chem. 2015, 177, 346-353.

237. Yang, C.; Wang, Y.; Wu, B.; Fang, J.; Li, S. Volatile compounds evolution of three table grapes with different flavor during and after maturation. Food Chem. 2011, 128, 823-830. 
238. Thissen, U., Coulier, L.; Overkamp, K.M., Jetten, J.; Werff, B.J.C.; Ven, T.; Werf, M.J. A proper metabolomics strategy supports efficient food quality improvement: A case study on tomato sensory properties. Food Qual. Pref. 2011, 22, 499-506.

239. Bianchi, F.; Mangia, A.; Mattarozzi, M.; Musci, M. Characterization of the volatile profile of thistle honey using headspace solid-phase microextraction and gas chromatography-mass spectrometry. Food Chem. 2011, 129, 1030-1036.

240. Lušić, D.; Koprivnjak, O.; Ćurić, D.; Sabatini, A.G.; Conte, L.S. Volatile Profile of Croatian Lime Tree (Tilia sp.), Fir Honeydew (Abies alba) and Sage (Salvia officinalis) Honey. Food Technol. Biotechnol. 2007, 45, 156-165.

241. Moniruzzaman, M.; Rodríguez, I.; Ramil, M.; Cela, R.; Sulaiman, S.A.; Gan, S.H. Assessment of gas chromatography time-of-flight accurate mass spectrometry for identification of volatile and semi-volatile compounds in honey. Talanta 2014, 129, 505-515.

242. Akil, E.; Castelo-Branco, V.N.; Costa, A.M.M.; Vendramini, A.L.A.; Calado, V.; Torres, A.G. Oxidative Stability and Changes in Chemical Composition of Extra Virgin Olive Oils After Short?Term Deep?Frying of French Fries. J. Am. Oil Chem. Soc. 2015, 92, 409-421.

243. Cecchi, T.; Alfei, B. Volatile profiles of Italian monovarietal extra virgin olive oils via HS-SPMEGC-MS: Newly identified compounds, flavors molecular markers, and terpenic profile. Food Chem. 2013, 141, 2025-2035.

244. Romero, I.; García-González, D.L.; Aparicio-Ruiz, R.; Morales, M.T. Validation of SPMEGCMS method for the analysis of virgin olive oil volatiles responsible for sensory defects. Talanta 2015, 134, 394-401.

245. Chumpolsri, W.; Wijii, N.; Boontakham, P.; Nimmanpipug P.; Sookwong, P.; Luangkamin, S.; Wongpornchai, S. Variation of Terpenoid Flavor Odorants in Bran of Some Black and White Rice Varieties Analyzed by GC $\times$ GC-MS. J. Food Nutr. Res. 2015, 3, 114-120.

246. Griglione, A.; Liberto, E.; Cordero, C.; Bressanello, D.; Cagliero, C.; Rubiolo, P.; Bicchi, C.; Sgorbini, B. High-quality Italian rice cultivars: Chemical indices of ageing and aroma quality. Food Chem. 2015, 172, 305-313.

247. Ho, C.W.; Aida, W.M.W.; Maskat, M.Y.; Osman, H. Optimization of headspace solid phase microextraction (HS-SPME) for gas chromatography mass spectrometry (GC-MS) analysis of aroma compound in palm sugar (Arenga pinnata). J. Food Compos. Anal. 2006, 19, 822-830.

248. Sanahuja,, A.B.; Santonja, M.R.; Teruel, N.G.; Carratalá, M.L.M.; Selva, M.C.G. Classification of Almond Cultivars Using Oil Volatile Compound Determination by HS-SPME-GC-MS. J. Am. Oil Chem. Soc. 2011, 88, 329-336.

249. D’Auria, M.; Mauriello, G.; Racioppi, R.; Rana, G.L. Use of SPME-GC-MS in the Study of Time Evolution of the Constituents of Saffron Aroma: Modifications of the Composition During Storage. J. Chromatogr. Sci. 2006, 44, 18-21.

250. Kim, N.Y.; Park, M.H.; Jang, E.Y.; Lee, J.H. Volatile Distribution in Garlic (Allium sativum L.) by Solid Phase Microextraction (SPME) with Different Processing Conditions. Food Sci. Biotechnol. 2011, 20, 775-782.

251. Fratini, G.; Lois, S.; Pazos, M.; Parisi, G.; Fratini, I.M.G.; Lois, S.; Pazos, M.; Parisi, G.; Medina, I. Volatile profile of Atlantic shellfish species by HS-SPME GC/MS. Food Res. Int. 2012, 48, 856-865. 
252. Xiao, Q.; Yu, C.; Xing, J.; Hu, B. Comparison of headspace and direct single-drop microextraction and headspace solid-phase microextraction for the measurement of volatile sulfur compounds in beer and beverage by gas chromatography with flame photometric detection. J. Chromatogr. A 2006, 1125, 133-137.

253. da Silva, G.C.; da Silva, A.A.S.; da Silva, L.S.N.; Godoy, R.L.O.; Nogueira, L.C.; Quitério, S.L.; Raices, R.S.L. Method development by GC-ECD and HS-SPME-GC-MS for beer volatile Analysis. Food Chem. 2015, 167, 71-77.

254. Franco-Luesma, E.; Ferreira, V. Quantitative analysis of free and bonded forms of volatile sulfurcompouds in wine. Basic methodologies and evidences showing theexistence of reversible cation-complexed forms. J. Chromatogr. A 2014, 1359, 8-15.

255. Fan, W.; Tsai, I.M.; Qian, M.C. Analysis of 2-aminoacetophenone by direct-immersion solidphase microextraction and gas chromatography-mass spectrometry and its sensory impact in Chardonnay and Pinot gris wines. Food Chem. 2007, 105, 1144-1150.

256. Gocmen, D.; Elston, A.; Williams, T.; Parish, M.; Rouseff, R.L. Identification of medicinal off-flavours generated by Alicyclobacillus species in orange juice using GC-olfactometry and GC-MS. Lett. Appl. Microbiol. 2005, 40, 172-177.

257. Bagheri, H.; Aghakhani, A.; Es-haghi, A. Sol-Gel-based SPME and GC-MS for Trace Determination of Geosmin in Water and Apple Juice Samples. Chromatographia 2007, 66, 779-783.

258. Iamanaka, B.T.; Teixeira, A.A.; Teixeira, A.R.R.; Vicente, E.; Frisvad, J.C.; Taniwaki, M.H.; Bragagnolo, N. Potential of volatile compounds produced by fungi to influence sensory quality of coffee beverage. Food Res. Int. 2014, 64, 166-170.

259. Jeleń, H.H.; Mildner-Szkudlarz, S.; Jasińska, I.; Wąsowicz, E. A Headspace-SPME-MS Method for Monitoring Rapeseed Oil Autoxidation. J. Am. Oil Chem. Soc. 2007, 84, 509-517.

260. Petersen, K.D.; Kleeberg, K.K.; Jahreis, G.; Fritsche, J. Assessment of the oxidative stability of conventional and high-oleic sunflower oil by means of solid-phase microextraction-gas chromatography. Int. J. Food Sci. 2012, 63, 160-169.

261. Petersen, K.D.; Kleeberg, K.K.; Jahreis, G.; Busch-Stockfisch, M.; Fritsche, J. Comparison of analytical and sensory lipid oxidation parameters in conventional and high-oleic rapeseed oil. Eur. J. Lipid Sci. Technol. 2012, 114, 1193-1203.

262. Petersen, K.D.; Jahreis, G.; Busch-Stockfisch, M.; Fritsche, J. Chemical and sensory assessment of deep-frying media alternatives for the processing of French fries. Eur. J. Lipid Sci. Technol. 2013, 115, 935-945.

263. Beltrán, A.; Ramos, M.; Grané, N.; Martín, M.L.; Garrigós, M.C. Monitoring the oxidation of almond oils by HS-SPME-GC-MS and ATR-FTIR: Application of volatile compounds determination to cultivar authenticity. Food Chem. 2011, 126, 603-609.

264. Panseri, S.; Soncin, S.; Chiesa, L.M.; Biondi, P.A. A headspace solid-phase microextraction gaschromatographic mass-spectrometric method (HS-SPME-GC/MS) to quantify hexanal in butter during storage as marker of lipid oxidation. Food Chem. 2011, 127, 886-889.

265. Achouri, A.; Boye, J.I.; Zamani, Y. Identification of volatile compounds in soymilk using solidphase microextraction-gas chromatography. Food Chem. 2006, 99, 759-766. 
266. Silcock, P.; Alothman, M.; Zardin, E.; Heenan, S.; Siefarth, C.; Bremer, P.J.; Beauchamp, J. Microbially induced changes in the volatile constituents of fresh chilled pasteurised milk during storage. Food Pack. Shelf Life 2014, 2, 81-90.

267. Wold, J.P.; Skaret, J.; Dalsgaard, T.K. Assessment of the action spectrum for photooxidation in full fat bovine milk. Food Chem. 2015, 179, 68-75.

268. Mikš-Krajnik, M.; Yoon, Y.J.; Yuk, H.G. Detection of Volatile Organic Compounds as Markers of Chicken Breast Spoilage Using HS-SPME-GC/MS-FASST. Food Sci. Biotechnol. 2014, 24, 361-372.

269. Duflos, G.; Moine, F.; Coin, V.M.; Malle, P. Determination of Volatile Compounds in Whiting (Merlangius merlangus) Using Headspace-Solid-Phase Microextraction-Gas ChromatographyMass Spectrometry. J. Chromatogr. Sci. 2005, 43, 304-312.

270. Robertson, R.F.; Jauncey, K.; Beveridge, M.C.M.; Lawton, L.A. Depuration rates and the sensory threshold concentration of geosmin responsible for earthy-musty taint in rainbow trout, Onchorhynchus mykiss. Aquaculture 2005, 245, 89-99.

271. Vinaixa, M.; Vergara, A.; Duran, C.; Llobet, E.; Badia, C.; Brezmes, J.; Vilanova, J.; Correig, X. Fast detection of rancidity in potato crisps using e-noses based on mass spectrometry or gas sensors. Sens. Actuat. B 2005, 106, 67-75.

272. Gallardo-Chacón, J.J.; Karbowiak, T. Sorption of 4-ethylphenol and 4-ethylguaiacol by suberin from cork. Food Chem. 2015, 181, 222-226.

273. Pizarro, C.; Pérez-del-Notario, N.; González-Sáiz, J.M. Multiple headspace solid-phase microextraction for eliminating matrix effect in the simultaneous determination of haloanisoles and volatile phenols in wines. J. Chromatogr. A 2007, 1166, 1-8.

274. Gómez-Ariza, J.L.; García-Barrera, T.; Lorenzo, F.; Beltrán, R. Use of multiple headspace solidphase microextraction and pervaporation for the determination of off-flavours in wine. J. Chromatogr. A 2006, 1112, 133-140.

275. Tait, E.; Perry, J.D.; Stanforth, S.P.; Dean, J.R. Bacteria detection based on the evolution of enzyme-generated volatile organic compounds: Determination of Listeria monocytogenes in milk samples. Anal. Chim. Acta 2014, 848, 80-87.

276. Bianchi, F.; Careri, M.; Mangia, A.; Mattarozzi, M.; Musci, M. Experimental design for the optimization of the extraction conditions of polycyclic aromatic hydrocarbons in milk with a novel diethoxydiphenylsilane solid-phase microextraction fiber. J. Chromatogr. A 2008, 1196-1197.

277. Zhang, Y.; Huang, X.; Yuan, D. Determination of benzimidazole anthelmintics in milk and honey by monolithic fiber-based solid-phase microextraction combined with high-performance liquid chromatography-diode array detection. Anal. Bioanal. Chem. 2015, 407, 557-567.

278. Kamalabadi, M.; Ghaemi, E.; Mohammadi, A.; Alizadeh, N. Determination of furfural and hydroxymethylfurfural from baby formula using headspace solid phase microextraction based on nanostructured polypyrrole fiber coupled with ion mobility spectrometry. Food Chem. 2015, 181, 72-77.

279. Sagratini, G.; Mañes, J.; Giardiná, D.; Damiani, P.; Picó, Y. Analysis of carbamate and phenylurea pesticide residues in fruit juices by solid-phase microextraction and liquid chromatography-mass spectrometry. J. Chromatogr. A 2007, 1147, 135-143. 
280. Lim, H.H.; Shin, H.S. Simple Determination of 4-Methylimidazole in Soft Drinks by Headspace SPME GC-MS. Chromatographia 2013, 76, 97-101.

281. Zuin, V.G.; Schellin, M.; Montero, L.; Yariwake, J.H.; Augusto, F.; Popp, P. Comparison of stir bar sorptive extraction and membrane-assisted solvent extraction as enrichment techniques for the determination of pesticide and benzoa.pyrene residues in Brazilian sugarcane juice. J. Chromatogr. A 2006, 1114, 180-187.

282. Wen, Y.; Wang, Y.; Feng, Y.Q. A simple and rapid method for simultaneous determination of benzoic and sorbic acids in food using in-tube solid-phase microextraction coupled with highperformance liquid chromatography. Anal. Bioanal. Chem. 2007, 388, 1779-1787.

283. Mei, M.; Huang, X.; Liao, K.; Yuan, D. Sensitive monitoring of benzoylurea insecticides in water and juice samples treated with multiple monolithic fiber solid-phase microextraction and liquid chromatographic analysis. Anal. Chim. Acta 2015, 860, 29-36.

284. Guillot, S.; Kelly, M.T.; Fenet, H.; Larroque, M. Evaluation of solid-phase microextraction as an alternative to the official method for the analysis of organic micro-pollutants in drinking water. J. Chromatogr. A 2006, 1101, 46-52.

285. Cai, L.; Gong, S.; Chen, M.; Wu, C. Vinyl crown ether as a novel radical crosslinked sol-gel SPME fiber for determination of organophosphorus pesticides in food samples. Anal. Chim. Acta 2006, 559, 89-96.

286. Poinot, P.; Qin, F.; Lemoine, M.; Yvon, V.; Ledauphin, J.; Gaillard, J.L. Study of current analytical strategies for the dual investigation of polycyclic aromatic hydrocarbons and benzene, toluene, ethylbenzene and xylene in apples. J. Food Compos. Anal. 2014, 35, 83-93.

287. Abdulra'uf, L.B.; Tan, G.H. Chemometric approach to the optimization of HS-SPME/GC-MS for the determination of multiclass pesticide residues in fruits and vegetables. Food Chem. 2015, 177, $267-273$.

288. Siripatrawan, U.; Harte, B.R. Solid phase microextraction/gas chromatography/mass spectrometry integrated with chemometrics for detection of Salmonella typhimurium contamination in a packaged fresh vegetable. Anal. Chim. Acta 2007, 581, 63-70.

289. Lei, F.F.; Huang, J.Y.; Zhang, X.N., Liu, X.J.; Li, X.J. Determination of Polycyclic Aromatic Hydrocarbons in Vegetables by Headspace SPME-GC. Chromatographia 2011, 74, 99-107.

290. Sapahin, H.A.; Makahleh, A.; Saad, B. Determination of organophosphorus pesticide residues in vegetables using solid phase micro-extraction coupled with gas chromatography-flame photometric detector. Arab. J. Chem. 2015, doi: 10.1016/j.arabjc.2014.12.001

291. Dong, C.; Zeng, Z.; Lia, X. Determination of organochlorine pesticides and their metabolites in radish after headspace solid-phase microextraction using calix4.arene fiber. Talanta 2005, 66, $721-727$.

292. Arisseto, A.P.; Vicente, E.; Ueno, M.S.; Toledo, M.C.F. Occurrence of furan in commercial samples of roasted coffee in Brazil. Food Addit. Contam. A 2012, 29, 1832-1839.

293. Liu, M.; Peng, Q.; Chen, Y.; Tang, Q.; Feng, Q. A rapid space-resolved solid-phase microextraction method as a powerful tool to determine contaminants in wine based on their volatility. Food Chem. 2015, 176, 12-16. 
294. Martins, J.; Esteves, C.; Limpo-Faria, A.; Barros, P.; Ribeiro, N; Simões, T.; Correia, M.; DelerueMatos, C. Analysis of six fungicides and one acaricide in still and fortified wines using solid-phase microextraction-gas chromatography/tandem mass spectrometry. Food Chem. 2012, 132, 630-636.

295. Pérez-Palacios, T.; Petisca, C.; Henriques, R.; Ferreira, I.M.P.L.V.O. Impact of cooking and handling conditions on furanic compounds in breaded fish products. Food Chem. Toxicol. 2013, $55,222-228$.

296. Jaffrès, E.; Lalanne, V.; Macé, S.; Cornet, J.; Cardinal, M.; Sérot, T.; Dousset, X.; Joffraud, J.J. Sensory characteristics of spoilage and volatile compounds associated with bacteria isolated from cooked and peeled tropical shrimps using SPME-GC-MS analysis. Int. J. Food Microbiol. 2011, 147, 195-202.

297. Bhattacharjee, P.; Panigrahi, S.; Lin, D.; Logue, C.M.; Sherwood, J.L.; Doetkott, C.; Marchello, M. A comparative qualitative study of the profile of volatile organic compounds associated with Salmonella contamination of packaged aged and fresh beef by HS-SPME/GC-MS. J. Food Sci. Technol. 2011, 48, 1-13.

298. Martin, D.; Ruiz, J. Analysis of polycyclic aromatic hydrocarbons in solid matrixes by solid-phase microextraction coupled to a direct extraction device. Talanta 2007, 71, 751-757.

299. Page, B.D.; Lacroix, G. Application of solid-phase microextraction to the headspace gas chromatographic analysis of halogenated volatiles in selected foods. J. Chromatogr. A 1993, 648, 199-211.

300. Shahidi, F.; Rubin, L.; D'Souza, L.A. Meat flavor volatiles: A review of the composition, techniques of analysis, and sensory evaluation. CRC Crit. Rev. Food Sci. Nutr. 1986, 24, 141-243.

301. Sostaric, T.; Boyce, M.C.; Spickett, E.E. Analysis of the volatile components in vanilla extracts and flavorings by solid-phase microextraction and gas chromatography. J. Agric. Food Chem. 2000, 48, 5802-5807.

302. Botondi, R.; DeSantis, D.; Bellincontro, A.; Vizovitis, K.; Mencarelli, F. Influence of ethylene inhibition by 1-methyl-cyclopropene on apricot quality, volatile production, and glycosidase activity of lowand high-aroma varieties of apricots. J. Agric. Food Chem. 2003, 51, 1189-1200.

303. Greger, V.; Schieberle, P. Characterization of the key aroma compounds in apricots (Prunus armeniaca) by application of the molecular sensory science concept. J. Agric. Food Chem. 2007, 55, 5221-5228.

304. Jeleń, H.H. Solid-Phase Microextraction in the Analysis of Food Taints and Off-Flavors. J. Chromatogr. Sci. 2006, 44, 399-415.

305. Melchert, H.U.; Pabel, E. Reliable identification and quantification of trichothecenes and other mycotoxins by electron impact and chemical ionization-gas chromatography-mass spectrometry, using an ion-trap system in the multiple mass spectrometry mode: Candidate reference method for complex matrices. J. Chromatogr. A 2004, 1056, 195-199.

306. Chan, D.; MacDonald, S.J.; Boughtflower, V.; Brereton, P. Simultaneous determination of aflatoxins and ochratoxin $\mathrm{A}$ in food using a fully automated immunoaffinity column clean-up and liquid chromatography-fluorescence detection. J. Chromatogr. A 2004, 1059, 13-16.

307. Tafuri, A.; Ferracane, R.; Ritieni, A. Ochratoxin A in Italian marketed cocoa products. Food Chem. 2004, 88, 487-494. 
308. Vale, P.; Sampayo, M.A. Esters of okadaic acid and dinophysistoxin-2 in Portuguese bivalves related to human poisonings. Toxicon 1999, 37, 1109-1121.

309. Hui, C.A.; Rudnick, D.; Williams, E. Mercury burdens in Chinese mitten crabs (Eriocheir sinensis) in three tributaries of southern San Francisco Bay, California, USA. Environ. Pollut. 2005, 133, $481-487$.

310. Vupputuri, S.; Longnecker, M.P.; Daniels, J.L.; Guo, X.; Sandler, D.P. Blood mercury level and blood pressure among US women: Results from the National Health and Nutrition Examination Survey 1999-2000. Environ. Res. 2005, 97, 195-200.

311. Storelli, M.M.; Storelli, A.; Giacominelli-Stuffler, R.; Marcotrigiano, G.O. Mercury speciation in the muscle of two commercially important fish, hake (Merluccius merluccius) and striped mullet (Mullus barbatus) from the Mediterranean sea: estimated weekly intake. Food Chem. 2005, 89, 295-300.

312. Meador, J.P.; Ernest, D.W.; Kagley, A.N. A comparison of the non-essential elements cadmium, mercury, and lead found in fish and sediment from Alaska and California. Sci. Total Environ. 2005, 339, 189-205.

313. Cubadda, F.; Raggi, A. Determination of cadmium, lead, iron, nickel and chromium in selected food matrices by plasma spectrometric techniques. Microchem. J. 2005, 79, 91-96.

314. Zhao, F.J.; Adams, M.L.; Dumont, C.; McGrath, S.P.; Chaudri, A.M.; Nicholson, F.A.; Chambers, B.J.; Sinclair, A.H. Factors affecting the concentrations of lead in British wheat and barley grain. Environ. Pollut. 2004, 131,461-468.

315. Sotelo, C.G.; Pineiro, C.; Perez-Martin, R.I. Denaturation of fish proteins during frozen storage: role of formaldehyde. Lebensm. Unters. For. 1995, 200, 14-23.

316. Badii, F.; Howell, N.K. Changes in the texture and structure of cod and haddock fillets during frozen storage. Food Hydrocol. 2002, 16, 313-319.

317. Bianchi, F.; Careri, M.; Corradini, C.; Musci, M.; Mangia, A. Innovative Method for Ultratrace Determination of Formaldehyde in Frozen Fish: SPME Extraction and GC-ITMS/MS Analysis. Curr. Anal. Chem. 2005, 1, 129-134.

318. Carro, A.M.; González, P.; Fajar, N.M.; Lorenzo, R.A.; Cela, R. Solid-phase micro-extraction procedure for the determination of 1,3-dichloro-2-propanol in water by on-fibre derivatisation with bis(trimethylsilyl) trifluoroacetamide. Anal. Bioanal. Chem. 2009, 394, 893-901.

319. Tumbiolo, S.; Gal, J.-F.; Maria, P.-C.; Zerbinati, O. SPME sampling of BTEX before GC/MS analysis: examples of outdoor and indoor air quality measurements in public and private sites. Ann. Chim. 2005, 95, 757-766.

320. Koziel, J.A.; Cai, L.; Wright, D.W.; Hoff, S.J. Solid-Phase Microextraction as a Novel Air Sampling Technology for Improved, GC-Olfactometry-Based Assessment of Livestock Odors. J. Chromatogr. Sci. 2006, 44, 451-457.

321. Kim, K.H.; Kim, D. A combination of Tedlar bag sampling and solid-phase microextraction for the analysis of trimethylamine in air: Relationship between concentration level and sample size. Microchem. J. 2009, 91, 16-20.

322. Ras, M.R.; Marcé, R.M.; Borrull, F. Solid-phase microextraction-Gas chromatography to determine volatile organic sulfur compounds in the air at sewage treatment plants. Talanta $\mathbf{2 0 0 8}$, 77, 774-778. 
323. Tassi, F.; Capecchiacci, F.; Buccianti, A.; Vaselli, O. Sampling and analytical procedures for the determination of VOCs released into air from natural and anthropogenic sources: A comparison between SPME (Solid Phase Micro Extraction) and ST (Solid Trap) methods. Appl. Chem. 2012, 27, 115-123.

324. Conde, F.J.; Afonso, A.M.; González, V.; Ayala, J.H. Optimization of an analytical methodology for the determination of alkyl- and methoxy-phenolic compounds by HS-SPME in biomass smoke. Anal. Bioanal. Chem. 2006, 385, 1162-1171.

325. Krüger, R.L.; Dallago, R.M.; do Nascimento Filho, I.; Di Luccio, M. Study of odor compounds in gaseous effluents generated during production of poultry feather and viscera meal using headspace solid phase microextraction. Environ. Monit. Assess. 2009, 158, 355-363.

326. Regueiro, J.; Garcia-Jares, C.; Llompart, M.; Lamas, J.P.; Cela, R. Development of a method based on sorbent trapping followed by solid-phase microextraction for the determination of synthetic musks in indoor air. J. Chromatogr. A 2009, 1216, 2805-2815.

327. Isetun, S.; Nilsson, U. Dynamic field sampling of airborne organophosphate triesters using solidphase microextraction under equilibrium and non-equilibrium conditions. Analyst 2005, 130, 94-98.

328. Miller, D.N.; Woodbury, B.L. A solid-phase microextraction chamber method for analysis of manure volatiles. J. Environ. Qual. 2006, 35, 2383-2394.

329. Yassaa, N.; Custer, T.; Song, W.; Pech, F.; Kesselmeier, J.; Williams, J. Quantitative and enantioselective analysis of monoterpenes from plant chambers and in ambient air using SPME. Atmos. Meas. Tech. 2010, 3, 1615-1627.

330. Menezes, H.C.; Cardeal, Z.L. Determination of polycyclic aromatic hydrocarbons from ambient air particulate matter using a cold fiber solid phase microextraction gas chromatography-mass spectrometry method. J. Chromatogr. A 2011, 1218, 3300-3305.

331. Hippelein, M. Analysing selected VVOCs in indoor air with solid phase microextraction (SPME): A case study. Chemosphere 2006, 65, 271-277.

332. Mocho, P.; Nicolle, J.; Desauziers, V. Modelling of adsorption kinetics and calibration curves of gaseous volatile organic compounds with adsorptive solid-phase microextraction fibre: Toluene and acetone for indoor air applications. Anal. Bioanal. Chem. 2008, 392, 97-104.

333 Cai, L.; Koziel, J.A.; Lo, Y.C.; Hoff, S.J. Characterization of volatile organic compounds and odorants associated with swine barn particulate matter using solid-phase microextraction and gas chromatography-mass spectrometry-olfactometry. J. Chromatogr. A 2006, 1102, 60-72.

334. Ding, Z.; Peng, S.; Xia, W.; Zheng, W.; Chen, X.; Yin, L. Analysis of Five Earthy-Musty Odorants in Environmental Water by HS-SPME/GC-MS. Int. J. Anal. Chem. 2014, 1-11.

335. Ma, K.; Zhang, J.N.; Zhao, M.; He, Y.J. Accurate analysis of trace earthy-musty odorants in water by headspace solid phase microextraction gas chromatography-mass spectrometry. J. Sep. Sci. 2012, 35, 1494-1501.

336. Sung, Y.H.; Li, T.Y.; Huang, S.D. Analysis of earthy and musty odors in water samples by solidphase microextraction coupled with gas chromatography/ion trap mass spectrometry. Talanta 2005, 65, 518-524. 
337. Díaz, A.; Ventura, F.; Galceran, M.T. Analysis of odorous trichlorobromophenols in water by in-sample derivatization/solid-phase microextraction GC/MS. Anal. Bioanal. Chem. 2006, 386, 293-298.

338. Lu, X.; Fan, C.; Shang, J.; Deng, J.; Yin, H. Headspace solid-phase microextraction for the determination of volatile sulfur compounds in odorous hyper-eutrophic freshwater lakes using gas chromatography with flame photometric detection. Microchem. J. 2012,104, 26-32.

339. Polo, M.; Garcia-Jares, C.; Llompart, M.; Cela, R. Optimization of a sensitive method for the determination of nitro musk fragrances in waters by solid-phase microextraction and gas chromatography with micro electron capture detection using factorial experimental design. Anal. Bioanal. Chem. 2007, 388, 1789-1798.

340. Cervera, M.I.; Beltran, J.; Lopez, F.J.; Hernandez, F. Determination of volatile organic compounds in water by head space-solid-phase microextraction gas chromatography coupled to tandem mass spectrometry with triple quadrupole analyzer. Anal. Chim. Acta 2011, 704, 87-97.

341. Badoil, L.; Benanou, D. Characterization of volatile and semivolatile compounds in waste landfill leachates using stir bar sorptive extraction-GC/MS. Anal. Bioanal. Chem. 2009, 393, 1043-1054.

342. Kos, G.; Ariya, P.A. Determination of a wide range of volatile and semivolatile organic compounds in snow by use of solid-phase micro-extraction (SPME). Anal. Bioanal. Chem. 2006, $385,57-66$.

343. Casas, V.; Llompart, M.; Garcia-Jares, C.; Cela, R.; Dagnac, T. Effects of sample pretreatment and storrage conditions in the determination of pyrethroids in water samples by solid-phase microextraction and gas chromatography-mass spectrometry. Anal. Bioanal. Chem. 2007, 387, 1841-1849.

344. Vázquez, P.P.; Mughari, A.R.; Galera, M.M. Application of solid-phase microextraction for determination of pyrethroids in groundwater using liquid chromatography with post-column photochemically induced fluorimetry derivatization and fluorescence detection. J. Chromatogr. A 2008, 1188, 61-68.

345. López Monzón, A.; Vega Moreno, D.; Torres Padron, M.E.; Sosa Ferrera, Z.; Santana Rodriguez, J.J. Solid-phase microextraction of benzimidazole fungicides in environmental liquid samples and HPLC-fluorescence determination. Anal. Bioanal. Chem. 2007, 387, 1957-1963.

346. Dong, C.; Zeng, Z.; Yang, M. Determination of organochlorine pesticides and their derivations in water after HS-SPME using polymethylphenylvinylsiloxane-coated fiber by GC-ECD. Water Res. 2005, 39, 4204-4210.

347. Raposo, J.L., Jr.; Ré-Poppi, N. Determination of organochlorine pesticides in ground water samples using solid-phase microextraction by gas chromatography-electron capture detection. Talanta 2007, 72, 1833-1841.

348. Padrón, M.E.T.; Ferrera, Z.S.; Rodríguez, J.J.S. Optimisation of solid-phase microextraction coupled to HPLC-UV for the determination of organochlorine pesticides and their metabolites in environmental liquid samples. Anal. Bioanal. Chem. 2006, 386, 332-340.

349. Brondi, S.H.G.; Rodrigues da Silva, J.C.; Lanças, F.M. Development and validation of a methodology for the determination of pesticides in water by SPME-LC/DAD. J. Liquid Chromatogr. Rel. Technol. 2005, 28, 2909-2919. 
350. Filho, A.M.; Neves dos Santos, F.; Pereira, P.A.P. Development, validation and application of a method based on DI-SPME and GC-MS for determination of pesticides of different chemical groups in surface and groundwater samples. Microchem. J. 2010, 96, 139-145.

351. Bagheri, H.; Ayazi, Z.; Babanezhad, E. A sol-gel-based amino functionalized fiber for immersed solid-phase microextraction of organophosphorus pesticides from environmental samples. Microchem. J. 2010, 94, 1-6.

352. GonÇalves, C.M.; Esteves da Silva, J.C.G.; Alpendurada, M.F. Evaluation of the Pesticide Contamination of Groundwater Sampled over Two Years from a Vulnerable Zone in Portugal. J. Agric. Food Chem. 2007, 55, 6227-6235.

353. Coelho, E.; Ferreira, C.; Almeida, C.M.M. Analysis of Polynuclear Aromatic Hydrocarbons by SPME-GC-FID in Environmental and Tap Waters. J. Braz. Chem. Soc. 2008, 19, 1084-1097.

354. Rianawati, E.; Balasubramanian, R. Optimization and validation of solid phase micro-extraction (SPME) method for analysis of polycyclic aromatic hydrocarbons in rainwater and stormwater. Phys. Chem. Earth 2009, 34, 857-865.

355. Wei, M.C.; Jen, J.F. Determination of polycyclic aromatic hydrocarbons in aqueous samples by microwave assisted headspace solid-phase microextraction and gas chromatography/flame ionization detection. Talanta 2007, 72, 1269-1274.

356. Zuazagoitia, D.; Millán, E.; Garcia, R. A Screening Method for Polycyclic Aromatic Hydrocarbons Determination in Water by Headspace SPME with GC-FID. Chromatographia 2007, 66, 773-777.

357. Luan, T.; Fang, S.; Zhong, Y.; Lin, L.; Chan, S.M.N.; Lan, C.; Tam, N.F.Y. Determination of hydroxy metabolites of polycyclic aromatic hydrocarbons by fully automated solid-phase microextraction derivatization and gas chromatography-mass spectrometry. J. Chromatogr. A 2007, 1173, 37-43.

358. Jaber, F.; Schummer, C.; Chami, J.A.; Mirabel, P.; Millet, M. Solid-phase microextraction and gas chromatography-mass spectrometry for analysis of phenols and nitrophenols in rainwater, as their t-butyldimethylsilyl derivatives. Anal. Bioanal. Chem. 2007, 387, 2527-2535.

359. Ho, H.P.; Lee, R.J.; Lee, M.R. Purge-assisted headspace solid-phase microextraction combined with gas chromatography-mass spectrometry for determination of chlorophenols in aqueous samples. J. Chromatogr. A 2008, 1213, 245-248.

360. Beceiro-González, E.; Guimaraes, A.; Alpendurada, M.F. Optimisation of a headspace-solid-phase micro-extraction method for simultaneous determination of organometallic compounds of mercury, lead and tin in water by gas chromatography-tandem mass spectrometry. J. Chromatogr. A 2009, 1216, 5563-5569.

361. López-Grimau, V.; Guadayol, J.M.; Griera, J.A.; Gutiérrez, M.C. Determination of non halogenated solvents in industrial wastewater using solid phase microextraction (SPME) and GC-MS. Lat. Am. Appl. Res. 2006, 36, 45-55.

362. Hudson, E.D.; Okuda, K.; Ariya, P.A. Determination of acetone in seawater using derivatization solid-phase microextraction. Anal. Bioanal. Chem. 2007, 388, 1275-1282.

363. Lee, M.R.; Chang, C.M.; Jianpeng, D. Determination of benzene, toluene, ethylbenzene, xylenes in water at sub-ng 1_1 levels by solid-phase microextraction coupled to cryo-trap gas chromatography-mass spectrometry. Chemosphere 2007, 69, 1381-1387. 
364. Lim, H.H.; Shin, H.S. Simple Determination of Acrolein in Surface and Drinking Water by Headspace SPME GC-MS. Chromatographia 2012, 75, 943-948.

365. Yang L.; Luan, T.; Lan, C. Solid-phase microextraction with on-fiber silylation for simultaneous determinations of endocrine disrupting chemicals and steroid hormones by gas chromatographymass spectrometry. J. Chromatogr. A 2006, 1104, 23-32.

366. Hernández, F.; Portolés, T.; Pitarch, E.; López, F.J. Target and Nontarget Screening of Organic Micropollutants in Water by Solid-Phase Microextraction Combined with Gas Chromatography/High-Resolution Time-of-Flight Mass Spectrometry. Anal. Chem. 2007, 79, 9494-9504.

367. Derouiche, A; Driss, M.R.; Morizur, J.P.; Taphanel, M.H. Simultaneous analysis of polychlorinated biphenyls and organochlorine pesticides in water by headspace solid-phase microextraction with gas chromatography-tandem mass spectrometry. J. Chromatogr. A 2007, 1138, 231-243.

368. Jochmann, M.A.; Yuan, Y.; Schmidt, T.C. Determination of volatile organic hydrocarbons in water samples by solid-phase dynamic extraction. Anal. Bioanal. Chem. 2007, 387, 2163-2174.

369. Shutao, W.; Yan, W.; Hong, Y.; Jie, Y. Preparation of a Carbon-Coated SPME Fiber and Application to the Analysis of BTEX and Halocarbons in Water. Chromatographia 2006, 63, 365-371.

370. Bagheri, H.; Aghakhani, A. Novel nanofiber coatings prepared by electrospinning technique for headspace solid-phase microextraction of chlorobenzenes from environmental samples. Anal. Methods 2011, 3, 1284-1289.

371. Avila, M.A.S.; Breiter, R.; Mott, H. Development of a simple, accurate SPME-based method for assay of VOCs in column breakthrough experiments. Chemosphere 2007, 66, 18-29.

372. Wang, J.X.; Jiang, D.Q.; Gu, Z.Y.; Yan, Y.P. Multiwalled carbon nanotubes coated fibers for solidphase microextraction of polybrominated diphenyl ethers in water and milk samples before gas chromatography with electron-capture detection. J. Chromatogr. A 2006, 1137, 8-14.

373. Laor, Y.; Koziel, J.A.; Cai, L.; Ravid, U. Chemical-Sensory Characterization of Dairy Manure Odor Using Headspace Solid-Phase Microextraction and Multidimensional Gas Chromatography Mass Spectrometry-Olfactometry. J. Air Waste Manage. Assoc. 2008, 58, 1187-1197.

374. Kim, H.; McConnell, L.L.; Millner, P. Comparison of odorous volatile compounds from fourteen different commercial composts using solid-phase microextraction. Trans. ASAE 2005, 48, 315-320.

375. Higashikawa, F.S.; Cayuela, M.L.; Roig, A.; Silva, C.A.; Sanchez-Monedero, M.A. Matrix effect on the performance of headspace solid phase microextraction method for the analysis of target volatile organic compounds (VOCs) in environmental samples. Chemosphere 2013, 93, 2311-2318.

376. Larreta, J.; Vallejo, A.; Bilbao, U.; Alonso, A.; Arana, G.; Zuloaga, O. Experimental design to optimise the analysis of organic volatile compounds in cow slurry by headspace solid-phase microextraction-gas chromatography-mass spectrometry. J. Chromatogr. A 2006, 1136, 1-9.

377. Banar, M.; Özkan, A.; Vardar, C. Characterization of an urban landfill soil by using physicochemical analysis and solid phase microextraction (SPME)-GC/MS. Environ. Monit. Assess. 2007, 127, 337-351. 
378. Cela, R.; Dagnac, T. Simultaneous determination of traces of pyrethroids, organochlorines and other main plant protection agents in agricultural soils by headspace solid-phase microextractiongas chromatography. J. Chromatogr. Sci. 2008, 1188, 154-163.

379. Bondarenko, S.; Spurlock, F.; Gan, J. Analysis of Pyrethroids in sediment pore water by solidphase microextraction. Environ. Toxicol. Chem. 2007, 26, 2587-2593.

380. Witt, G.; Liehr, G.A.; Borck, D.; Mayer, P. Matrix solid-phase microextraction for measuring freely dissolved concentrations and chemical activities of PAHs in sediment cores from the western Baltic Sea. Chemosphere 2009, 74, 522-529.

381. Rocha, M.J.; Ferreira, P.C.; Reis, P.A.; Cruzeiro, C.; Rocha, E. Determination of Polycyclic Aromatic Hydrocarbons in Coastal Sediments from the Porto Region (Portugal) by MicrowaveAssisted Extraction, Followed by SPME and GC-MS. J. Chromatogr. Sci. 2011, 49, 695-701.

382. Hawthorne, S.B.; Grabanski, C.B.; Miller, D.J.; Kreitinger, J.P. Solid-phase microextraction measurement of parent and alkyl polycyclic aromatic hydrocarbons in milliliter sediment pore water samples and determination of KDOC values. Environ. Sci. Technol. 2005, 39, 2795-2803.

383. Shen, G.; Huang, J.; Yu, G. Measurement of the free concentrations of alkyl phenols and bisphenol A to determine their biodegradation kinetics by activated sludge. Chin. Sci. Bull. 2007, 52, 27662770.

384. Zhou, J.; Yang, F.; Cha, D.; Zeng, Z.; Xu, Y. Headspace solid-phase microextraction with novel sol-gel permethylated-ß-cyclodextrin/hydroxyl-termination silicone oil fiber for determination of polybrominated diphenyl ethers by gas chromatography-mass spectrometry in soil. Talanta $\mathbf{2 0 0 7}$, $73,870-877$.

385. Campillo, N.; Peñalver, R.; Hernández-Córdoba, M. Solid-phase microextraction combined with gas chromatography and atomic emission detection for the determination of cyclopentadienylmanganese tricarbonyl and (methylcyclopentadienyl)manganese tricarbonyl in soils and seawaters. J. Chromatogr. A 2007, 1173, 139-145.

386. Drescher, S.R.; Brown, S.D. Solid phase microextraction-gas chromatographic-mass spectrometric determination of nitrous oxide evolution to measure denitrification in estuarine soils and sediments. J. Chromatogr. A 2006, 1133, 300-304.

387. Bicchi, C.; Cordero, C.; Liberto, E.; Rubiolo, P.; Sgorbini, B. Automated headspace solid-phase dynamic extraction to analyse the volatile fraction of food matrices. J. Chromatogr. A 2004, 1024, $217-226$.

388. Grosch, W. Evaluation of the Key Odorants of Foods by Dilution Experiments, Aroma Models and Omission. Chem. Senses 2001, 26, 533-545.

389. Schieberle, P. New Developments in Methods for Analysis of Volatile Flavor Compounds and their Precursors. In Characterization of Food: Emerging Methods; Goankar, A.G., Ed.; Elsevier Science B.V.: Amsterdam, The Netherlands, 1995; pp. 403-433.

(C) 2015 by the authors; licensee MDPI, Basel, Switzerland. This article is an open access article distributed under the terms and conditions of the Creative Commons Attribution license (http://creativecommons.org/licenses/by/4.0/). 